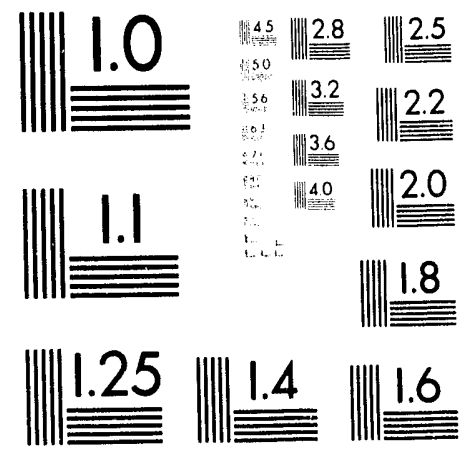



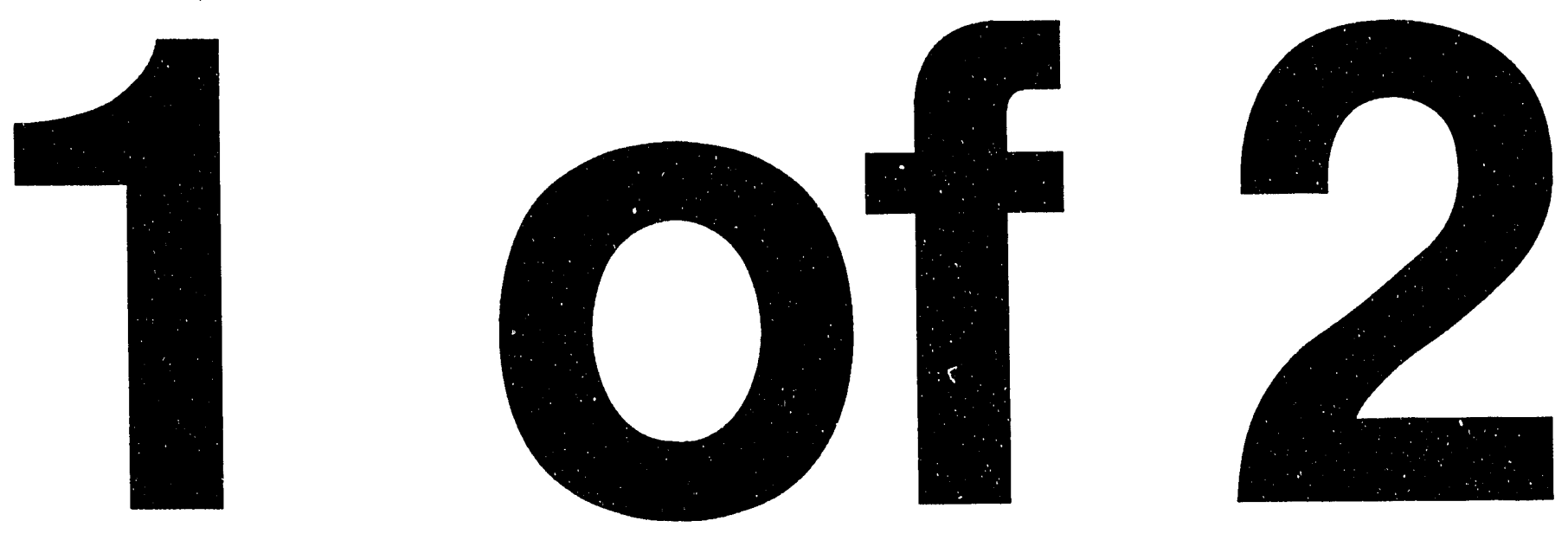


\title{
Hydrogen Separation by Ceramic Membranes in Coal Gasification
}

\author{
Final Report
}

G.R. Gavalas

Work Performed Under Contract No.: DE-AC21-90MC26365

\author{
For \\ U.S. Department of Energy \\ Office of Fossil Energy \\ Morgantown Energy Technology Center \\ P.O. Box 880 \\ Morgantown, West Virginia 26507-0880
}

\section{By}

California Institute of Technology

Pasadena, California 91125 


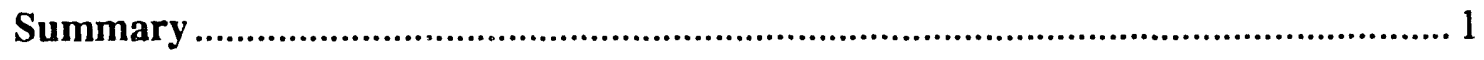

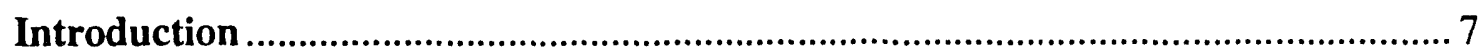

Chapter 1: Membrane Preparation and Characterization.................................... 12

1.1. Introduction ........................................................................... 13

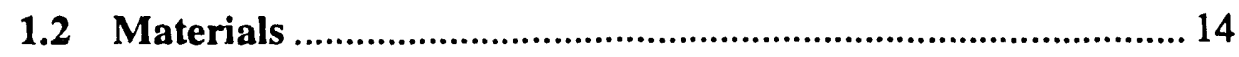

1.3 Apparatus and Procedure ...................................................... 15

1.4 Results of Membrane Preparation .......................................... 17

1.5 Membrane Characterization ..................................................... 20

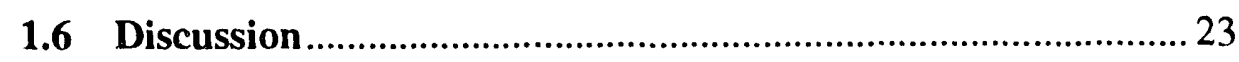

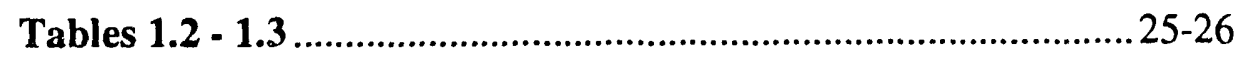

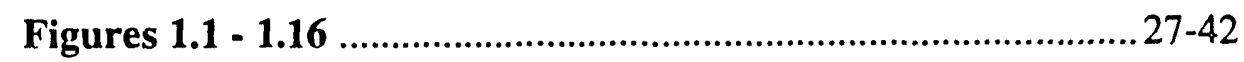

Chapter 2: Membrane Stability Testing ................................................................. 43

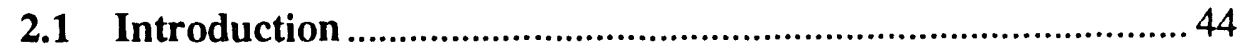

2.2 Apparatus and Procedure …………………………................. 45

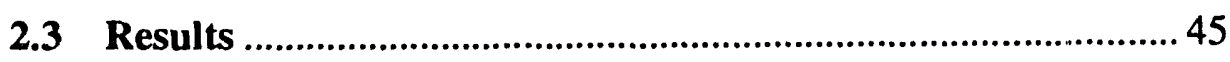

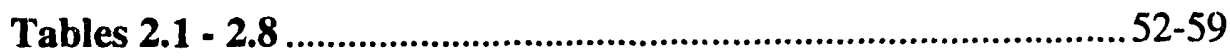

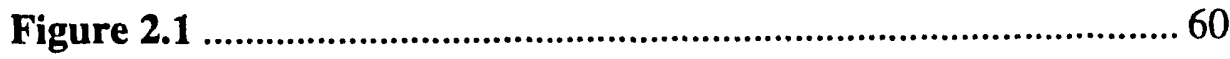

Chapter 3: Analysis and Economic Evaluation of $\mathrm{SiO}_{2}$ Membrane Application to Hydrogen Production from Coal Gas ......................... 61

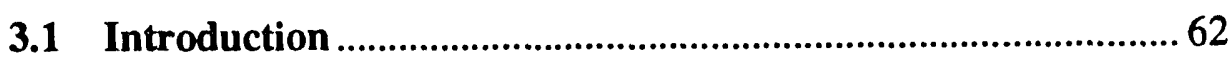

3.2 Description of the Membrane Shift Reactor ................................62

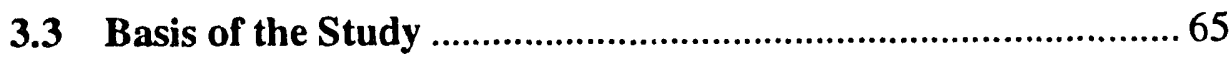

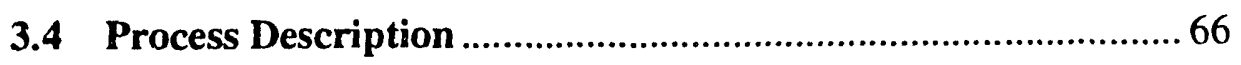

3.5 Comparison of Processes ………………………………........ 70

3.6 Process Optimization ............................................................... 74

3.7 Summary and Conclusions ......................................................

Figures 3.1 - 3.2 ........................................................................ $76-77$

Appendix: Model of the Membrane Shift Reactor ........................ 80

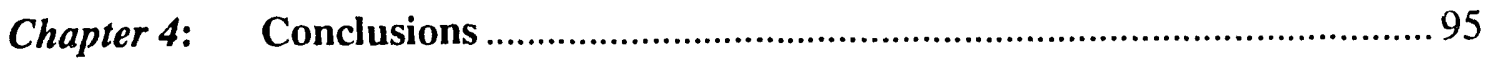




\section{Summary}

The general objective of this project was to develop hydrogen permselective membranes for hydrogen production from coal gas. The project consisted of the following tasks: (i) membrane preparation and characterization, (ii) membrane stability testing, and (iii) analysis and economic evaluation of a membrane-assisted ammonia from coal process.

\section{Membrane Preparation and Characterization}

Several oxides $\left(\mathrm{SiO}_{2}, \mathrm{TiO}_{2}, \mathrm{Al}_{2} \mathrm{O}_{3}, \mathrm{~B}_{2} \mathrm{O}_{3}\right)$ in dense (or nonporous) form were identified to be permselective to hydrogen at elevated temperatures. To obtain reasonable permeance it is necessary that the membrane consists of a thin selective layer of the dense oxide supported on or within the pores of a porous support tube (or plate). Early in the project we chose porous Vycor tubes ( $5 \mathrm{~mm} \mathrm{ID,} 7 \mathrm{~mm}$ OD, $40 \AA$ mean pore diameter) supplied by Corning Inc. as the membrane support. To form the permselective layer $\left(\mathrm{SiO}_{2}, \mathrm{TiO}_{2}, \mathrm{Al}_{2} \mathrm{O}_{3}, \mathrm{~B}_{2} \mathrm{O}_{3}\right)$ we employed chemical vapor deposition using the reaction of the chloride $\left(\mathrm{SiCl}_{4}\right.$, etc.) vapor and water vapor at high temperatures.

Deposition of the selective layer was carried out in a simple concentric tube reactor comprising the porous support tube surrounded by a wider concentric quartz tube and placed in an electrically heated split tube furnace. In one deposition geometry (the opposing reactants or two-sided geometry) the chloride vapor in nitrogen carrier was passed through the inner tube while the water vapor also in nitrogen carrier was passed in the same direction through the annulus between the two tubes. In the other (two-sided) geometry the chloride-containing stream and the water-containing stream were both passed through the inner tube or both through the annulus.

During deposition the flow of reactants was interrupted every few minutes to measure, in situ, the membrane permeance to hydrogen, nitrogen and occasionally other 
gases. Once the permeance to nitrogen decreased by a factor of at least 100 , deposition was terminated and the membrane stored for stability testing or microscopy.

Deposition of $\mathrm{SiO}_{2}$ membranes were carried out initially in the opposing reactants geometry. The temperature was $700-800^{\circ} \mathrm{C}$, the pressure atmospheric and the composition of the two reactant streams was $30 \% \mathrm{SiCl}_{4}-\mathrm{N}_{2}$ and $7 \% \mathrm{H}_{2} \mathrm{O}-\mathrm{N}_{2}$, respectively. The membrane permeance to hydrogen at $450^{\circ} \mathrm{C}$ was as high as $0.1 \mathrm{~cm}^{3} / \mathrm{cm}^{2}$-min-atm and the $\mathrm{H}_{2}: \mathrm{N}_{2}$ permeance ratio was in the range $1000-5000$.

Deposition of $\mathrm{SiO}_{2}$ membranes in the one-sided geometry was much faster and produced considerably higher hydrogen permeance, as high as $0.35 \mathrm{~cm}^{3} / \mathrm{cm}^{2}$-min-atm, but lower $\mathrm{H}_{2}: \mathrm{N}_{2}$ permeance ratio (200-1000).

Titanium oxide and aluminum oxide membranes could only be produced in the opposing reactants geometry. The $\mathrm{TiO}_{\text {: embranes had slightly lower hydrogen }}$ permeance and much lower selectivity than $\ldots: \mathrm{SiO}_{2}$ membranes prepared in the opposing reactant geometry. The $\mathrm{Al}_{2} \mathrm{O}_{3}$ membranes also had lower permeance and selectivity than the $\mathrm{SiO}_{2}$ membranes.

Boron oxide membranes were prepared in both the opposing and one-sided geometries. Deposition could take place at temperatures as low as $100^{\circ} \mathrm{C}$ and the membrane permeance at temperatures up to $450^{\circ} \mathrm{C}$ was as high as $0.2 \mathrm{~cm}^{3} / \mathrm{cm}^{2}$-min-atm. However, upon heating above $500^{\circ} \mathrm{C}$, the membrane permeance decreased sharply. Moreover, when these membranes were exposed to humid laboratory air for extended periods, they developed microcracks and lost their selectivity.

In view of the superior properties of the silica membranes, after the initial experiments work continued only with these membranes. The purpose of the later deposition studies was to explore means of increasing membrane permeance. To this end deposition was carried out with support tubes of smaller pore diameter $(25 \AA)$ and thinner wall $(0.35 \mathrm{~mm})$ and with two other silicon precursors, hexachloro-disiloxane, and 
octachloro-trisiloxane. Membranes with hydrogen permeance as high as $0.52 \mathrm{~cm}^{3} / \mathrm{cm}^{2}$ min-atm at $450^{\circ} \mathrm{C}$ were prepared with these support tubes and silica precursors.

In addition to measuring membrane permeance to different gases, membrane characterization was carried out by scanning electron microscopy (SEM) and electron microprobe analysis (EMA). SEM and EMA of $\mathrm{SiO}_{2}, \mathrm{TiO}_{2}, \mathrm{Al}_{2} \mathrm{O}_{3}$ membranes prepared by opposing reactants deposition revealed an asymmetric deposit layer consisting of a slowly rising section from the side of the chloride flow leading to a region of maximum density followed by a steep decline. By contrast, the density profile of the membranes prepared by one-sided deposition had their maximum at the boundary (the inner cylindrical surface) and was monotonically declining. In both types of membranes, the selectivity is due to the region around the peak of the deposit layer where Knudsen diffusion is completely interrupted and transport takes place by activated diffusion through the oxide network. The thickness of this region could not be measured accurately but was in the range $0.1-1 \mu \mathrm{m}$. The remaining and much thicker part of the deposit layer $(10-50 \mu \mathrm{m})$ which involves partially plugged pores has an adverse effect on permeance without contributing much to selectivity.

\section{Membrane Stability Testing}

The silica membranes prepared as described above were subjected to a variety of thermal and hydrothermal treatments to test their stability under conditions pertinent to catalytic water gas shift reaction of coal gas. Immediately after preparation the membranes were annealed in dry nitrogen at $700-750^{\circ} \mathrm{C}$ for about 12 hours. For membranes produced in the one-sided geometry this dry annealing resulted in a slight decline of hydrogen permeance (less than 10\%) and a significant decline of nitrogen permeance (by a factor 2-5) such that the $\mathrm{H}_{2}: \mathrm{N}_{2}$ selectivity improved.

One group of membranes were subjected to heating at $500-600^{\circ} \mathrm{C}$ in a pressure vessel containing $22 \% \mathrm{H}_{2}, 27 \% \mathrm{CO}, 32 \% \mathrm{H}_{2} \mathrm{O}, 18 \% \mathrm{CO}_{2}, 1 \% \mathrm{H}_{2} \mathrm{~S}$ at $10-12$ atmospheres 
total pressure. After four days such hydrothermal treatment, all membranes prepared in the opposing reactants geometry failed (c: led). The membranes prepared in the one-sided geometry did not fail but underwent $s$... ificant decline in their hydrogen permeance from about 0.2 to about $0.05 \mathrm{~cm}^{3} / \mathrm{cm}^{2}$-min-atm.

A second group of membranes of somewhat higher initial permeance, all prepared with one-sided deposition, were treated hydrothermally at $550^{\circ} \mathrm{C}$ for $2 \mathrm{i}$ days in a gas containing $32 \% \mathrm{H}_{2} \mathrm{O}-\mathrm{N}_{2}$ at 10 atmospheres total pressure. Initially the hydrogen permeance declined rapidly after which it declined slowly stabilizing to a value about three to four times below its initial value.

A third group of membranes prepared in the one-sided geometry but with different substrate tubes and silica precursors (see previous subsection) were treated hydrothermally under the same conditions as the second group for 13 days. These membranes also underwent a decline of hydrogen permeance by about a factor of four and a decline of the nitrogen permeance by a somewhat larger factor.

\section{Process Analysis and Economic Evaluation}

Kinetics Technology International (KTI), San Dimas, California Office, carried out analysis and economic evaluation of a membrane-assisted and a conventional process of ammonia from coal. This process was chosen as an important potential use of hydrogen from coal gasification, in order to evaluate the commercial potential of the silica membranes. The analysis and evaluation was based on the membrane properties measured at Caltech, a shift reactor model developed at Caltech, and cost estimates of Vycor tubing provided by Corning Inc.

In comparing the membrane-assisted and the conventional process, only the hydrogen production section (water gas shift unit and separation unit) was different for the two - scesses and had to be considered. The coal gasifier and hot gas cleanup, the ammunia production unit, and the power generation unit were the same in both processes. 
For the purpose of this study the coal gas was assumed to have composition typical of an oxygen-blown KRW gasifier converting about 1000 ton per day low sulfur $(0.4 \%)$ coal. Both the membrane and the conventional process use the same amount and composition of coal gas and produce purified hydrogen equivalent to 560 ton per day ammonia. Both processes produce electric power as well as ammonia by using part of the coal gas in a gas turbine-steam turbine dual cycle. Much of the power generated is used for the internal needs of the process, most of it for driving the ammonia compressors and the combustion air compressors. However, there is some net power produced by both processes. Although the two processes receive the same amount of coal gas and produce the same amount of ammonia they differ in net power production and in capital cost because they employ different unit operations and equipment.

The conventional process adds a large amount of steam to the coal gas such that after two successive catalytic shift stages (high temperature and low temperature) the conversion of $\mathrm{CO}$ is $99+\%$. Pure hydrogen with only traces of $\mathrm{CO}, \mathrm{CO}_{2}, \mathrm{CH}_{4}$ and $\mathrm{H}_{2} \mathrm{~S}$ is produced by pressure swing adsorption (PSA) and conducted to the ammonia plant. The net power production in this process was estimated to be $36.6 \mathrm{MW}$.

The membrane-assisted process requires much less steam to achieve high conversion for the shift reaction. The membrane reactor used in this process consists of three high temperature shift reactor units, each followed by a membrane unit and a heat exchanger. The permeate gas is high purity hydrogen which after methanation (to convert the few tens of a percent of $\mathrm{CO}$ and $\mathrm{CO}_{2}$ to methane) is used in the ammonia plant. The nonpermeate gas containing significant fractions of hydrogen, carbon dioxide and methane (deriving from the coal gas) is fed directly to the combined cycle power generation. The net power production in the membrane-assisted process was estimated to be $44.4 \mathrm{MW}$.

To obtain a cost estimate for the membrane it was assumed that each of the three membrane units consists of series-parallel combination of several thousand membrane modules. Each membrane module in turn consists of several thousand membrane 
capillaries sealed in parallel to an endplate and enclosed in a metal casing. The capillaries were assumed to have $0.5 \mathrm{~mm} \mathrm{ID}, 0.7 \mathrm{~mm}$ OD and hydrogen permeance at $500^{\circ} \mathrm{C}$ of 0.1 $\mathrm{cm}^{3} / \mathrm{cm}^{2}$-min-atm in accordance with the values measured after hydrothermal treatment.

On the basis of the above parameters, the membrane area required by the process was calculated to be $150,000 \mathrm{~m}^{2}$, or 19.3 tons of capillaries. The cost of the membrane units is difficult to estimate because Vycor tubes below $5 \mathrm{~mm}$ ID are not produced commercially. However, some very crude estimates made by Corning Inc. placed the cost at 300-600 dollars per pound. Using the figure of $\$ 500 / \mathrm{lb}$ and including some additional cost for the CVD treatment and the fabrication of modules and larger units, yielded a cost of about $\$ 24$ million for the membrane units. Compared to this membrane cost, the conventional process requires only $\$ 6$ million for the PSA unit. All other equipment are the same for both nrocesses. Thus, the membrane-assisted process has $24-6=\$ 18$ million extra cost while producing 7.8 MW extra power. With the price of power between $2 \mathrm{ct}$ and $5 \mathrm{ct}$ per $\mathrm{kWhr}$, the extra power would generate $\$ 1.36$ million to $\$ 3.4$ million annually. With these figures the extra cost for the membrane would require 5 to 13 years to be recovered.

The membrane process would become competitive if the hydrogen permeance could be increased by a factor of 3 to $0.3 \mathrm{~cm}^{3} / \mathrm{cm}^{2}$-min-atm so that the extra membrane cost would be reduced to $8-6=\$ 2$ million and the recovery period to $0.6-1.5$ years. All these estimates are subject to considerable uncertainty due to the lack of reliable costs for the individual membrane capillaries and the fabrication of membrane modules. Further work is clearly indicated on increasing membrane permeance, developing techniques for making multi-tube modules, and generating reliable cost estimates for tubes and modules. 


\section{Introduction}

Coal gasification is a promising alternative to steam reforming of natural gas as a source of hydrogen for the synthesis of ammonia and other chemicals. The hydrogen consumed in coal conversion to liquid fuels could also be derived from the gasification of coal or coal residues. In either of these uses of coal the separation of hydrogen from coal gas can be carried out by established processes like acid gas removal followed by pressure swing adsorption. However, membrane separation is a promising alternative having the potential of higher energy efficiency and simpler process flowsheet. To realize these potential advantages the separation must be carried out at elevated temperatures necessitating the use of inorganic membranes. Separation of hydrogen sufficiently pure for ammonia or other chemical synthesis requires membranes of very high selectivity, while separation of less pure hydrogen for use in coal liquefaction is feasible with less selective membranes. In the case of hydrogen production for chemical synthesis the use of hydrogen permselective inorganic membranes permits the integration of hydrogen separation and catalytic shift reaction in a membrane reactor in order to simplify the flowsheet and improve the economics of the process.

Both mesoporous (2-50 nm diameter) and nonporous inorganic membranes have been investigated from the standpoint of gas separations. Japanese investigators ${ }^{1-4}$ have studied the separation of gases by porous glass membranes with pore of size $30-50 \AA$. Membranes with similar pore size were made by sol gel techniques depositing a coating of mesoporous $\gamma-\mathrm{Al}_{2} \mathrm{O}_{3}$ on macroporous $\alpha-\mathrm{Al}_{2} \mathrm{O}_{3}$ and are available commercially as filters for

liquids purification. ${ }^{5-10}$ Reference $^{4}$ describes the application of mesoporous (30-50 $\AA$ ) silica glass membranes and $\gamma-\mathrm{Al}_{2} \mathrm{O}_{3}$ membranes to the catalytic dissociation of $\mathrm{H}_{2} \mathrm{~S}$ with sirultaneous $\mathrm{H}_{2}$ separation. 
Governed by Knudsen diffusion, the separation selectivities of microporous membranes are generally too low for membrane-reactor applications. For example, the ratio of Knudsen diffusivities for the $\mathrm{H}_{2}: \mathrm{CO}$ pair is only 3.74. Only activated diffusion through nonporous or molecular sieve materials can provide higher selectivities. Palladium and its alloys are known to be highly and selectively permeable to hydrogen and have been studied in connection with certain dehydrogenation reactions. The high cost of palladium and its sensitivity to poisoning by sulfur are obstacles to its applications to coal gas processing. Certain base metals (V, e.g.) are also semipermeable to hydrogen. Currently metal membranes are being developed under DOE funding for application to the water gas shift reaction and the conversion of hydrogen sulfide to elemental sulfur. ${ }^{11}$ These composite membranes consist $n$ ? 2 vanadium sheet surrounded at both sides by a $\mathrm{SiO}_{2}$ interdiffusion layer and an outer layer of a noble metal (e.g. $\mathrm{Pt}$ ). The role of the noble metal is to catalyze the dissociation of molecular hydrogen to atomic hydrogen as required for dissolution and transport through the $\mathrm{V}$ layer. Considerations of membrane stability and cost will ultimately determine the economic viability of these membranes.

Besides metals, silica glass is known to be highly selective for hydrogen permeation. ${ }^{12}$ For example, at $500^{\circ} \mathrm{C}$ the permeability of hydrogen through Vycor glass is more than four orders of magnitude higher than that of $\mathrm{O}_{2}$. This selectivity towards hydrogen is due to the relatively easy permeation of the small hydrogen molecule through the glass network with other gases $\left(\mathrm{O}_{2}, \mathrm{~N}_{2}, \mathrm{CO}\right.$, etc.) permeating extremely slowly. That low permeation rate makes $\mathrm{SiO}_{2}$ unsuitable for separations other than those involving hydrogen. The permeation rate depends on the composition of the glass and increases rapidly with temperature. To the best of our knowledge nonporous silica glass has not been studied before as a material for gas separations although, in addition to its high selectivity, it is easy to fabricate in a membrane configuration. The practical application of $\mathrm{SiO}_{2}$ membranes requires that they possess adequate permeation rates as well as good selectivity. These two requirements can be met by preparing the membrane in composite 
form, consisting of a thin layer of the dense oxide (silica, etc.) supported on a porous tube or plate--tube being the more practical geometry.

Deposition of silica and other oxide layers on porous support tubes or plates can be carried out by liquid phase or gas phase techniques. The oxide layers deposited by liquid phase techniques are porous, for driving off a solvent or a reaction product during drying and calcination leaves behind a porous structure. Gas phase techniques--chemical vapor deposition (CVD)--can, in principle, produce a dense (nonporous) deposit layer because the reaction products are removed during deposition and no drying or calcination steps are necessary.

Dense silica membranes were first prepared at Caltech using the oxidation of silane $\left(\mathrm{SiH}_{4}\right)$. The reaction was carried out in the opposing reactants geometry, i.e. passing one reactant $\left(\mathrm{SiH}_{4}\right)$ through the bore of the porous Vycor tube, and the other $\left(\mathrm{O}_{2}\right)$ past the outside surface of the tube; both at atmospheric pressure. The reactants diffuse and react within a certain region within the porous matrix. Once the open pore paths are interrupted, the reaction essentially ceases and the resulting nonporous layer provides the desired membrane function. The results of this work utilizing $\mathrm{SiH}_{4}$ oxidation have been reported earlier. ${ }^{13-15}$

Silica deposition by $\mathrm{SiH}_{4}$ oxidation is limited to a relatively narrow temperature window, $400-500^{\circ} \mathrm{C}$. Below $400^{\circ} \mathrm{C}$ the reaction is too slow producing a thick layer, therefore low membrane permeance. Above $500^{\circ} \mathrm{C}$ silane pyrolysis becomes significant producing elemental silicon over a wide region of the porous support, therefore again resulting in very low permeance. When the silica membranes produced by $\mathrm{SiH}_{4}$ oxidation was heated at temperatures above $450^{\circ} \mathrm{C}$ in the presence of water vapor they suffered a sharp decline of permeance and often a decline of selectivity as well. When water vapor was added to the $\mathrm{SiH}_{4}$ and $\mathrm{O}_{2}$ reactants during CVD, subsequent exposure to high temperature and water vapor caused loss of hydrogen permeance but improved the selectivity somewhat. 
Chemical vapor deposition of silica can be carried out by hydrolysis of silicon tetrachloride $\left(\mathrm{SiCl}_{4}\right)$ as well as by oxidation of $\mathrm{SiH}_{4}$. Silicon tetrachloride does not decompose thermally like $\mathrm{SiH}_{4}$, therefore the hydrolysis can be carried out at temperatures as high as $800^{\circ} \mathrm{C}$, above which, however, the porous structure of the Vycor support may start collapsing. Increasing the reaction temperature can, in principle, produce thinner deposit layers and, hence, higher membrane permeance. Besides its higher thermal stability, silicon tetrachloride is not flammable, unlike $\mathrm{SiH}_{4}$, and hence safer to handle. It is also quite inexpensive being an intermediate in the production of silicon and silicones. However, it is corrosive in the presence of humidity.

In addition to $\mathrm{SiCl}_{4}$, certain other volatile chlorides can be hydrolyzed in the gas phase to the respective oxides. These include $\mathrm{TiCl}_{4}, \mathrm{AlCl}_{3}$, and $\mathrm{BCl}_{3}$. Boron oxide $\left(\mathrm{B}_{2} \mathrm{O}_{3}\right)$ is amorphous and is known to be hydrogen permselective and have higher hydrogen permeability than $\mathrm{SiO}_{2}$. The other two oxides $\mathrm{TiO}_{2}$ and $\mathrm{Al}_{2} \mathrm{O}_{3}$ had not been investigated as hydrogen permselective materials.

This project was initiated with the objective to (i) prepare membranes of $\mathrm{SiO}_{2}$, $\mathrm{TiO}_{2}, \mathrm{Al}_{2} \mathrm{O}_{3}$ and $\mathrm{B}_{2} \mathrm{O}_{3}$ supported on porous $\mathrm{Vycor}$ tubes by the hydrolysis of the respective chlorides, (ii) characterize the morphology and permeation properties of these membranes, (iii) test the membrane stability in contact with coal gas under conditions typical of the water gas shift reaction, (iv) develop a membrane reactor model for the water gas shift reaction and $(v)$ conduct an economic analysis of membrane application to hydrogen production from coal gas.

This report is organized in four chapters. Chapter 1 describes the work on membrane preparation and characterization. Chapter 2 describes the stability testing. Chapter 3 is the process analysis and evaluation carried out by KTI, but also includes an appendix on membrane reactor modeling. The final Chapter 4 contains the general conclusions. 


\section{Literature Cited}

1. Kameyama, T., Dokiya, M., Yokokawa, H. and Fukuda, K. Ind. Engng. Chem. Fundam. 20, 97 (1981).

2. Shindo, Y., Hakuta, T., Yoshitome, H. and Inoue, H., Chem. Engng. Japan 17, 650 (1984).

3. Shindo, Y., Hakuta, T., Yoshitome, H. and Inoue, H., J. Chem. Engng. Japan 18, 485 (1985).

4. Haraya, K., Shindo, Y., Hakuda, T and Yoshitome, H., J. Chem. Engng. Japan 19, 186 (1986).

5. Leenaars, A. F. M. and Burggraaf, A. J., J. Colloid Interface Sci. 105, 27 (1978).

6. Keizer, K., Leenaars, A. F. M. and Burggraaf, A. J., in The Science of Ceramics, Vol. 12, Ceramurgica, Faenza, 1984.

7. Chu, P.-Y. and Clark, D. E., Advanced Ceramic Materials 3, 249 (1988).

8. Okubo, T., Watanabe, M., Kusakabe, K. and Morrooka, S., J. Materials Sci. 25, 4822 (1990).

9. Okubo, T., Haruta, K., Kusakabe, K. and Morooka, S., J. Membrane Sci. 59, 73 (1991).

10. Cini, P., Blaha, S. R., Harold, M. P. and Venkataraman, K., J. Membrane Sci. 55, 199 (1991).

11. Edlund, D. J., abstract of a paper presented at the 12th annual gasification and gas stream cleanup systems contractors review meeting, Morgantown Energy Technology Center, September 15-17, 1992.

12. Boyd, D. C. and Thompson, D. A., "Glass" Encyclopedia of Chemical Technology 11,807 (1980).

13. Gavalas, G. R., Megiris, C. E. and Nam, S. W., Chem. Eng. Sci. 441829 (1989).

14. Nam, S. W. and Gavalas, G. R., AIChE Symp. Ser. 85, 68 (1989).

15. Gavalas, G. R. and Megiris, C. E., U.S. Patent 4,902,307, 1990. 


\section{Chapter 1}

Membrane Preparation and Characterization 


\subsection{Introduction}

This chapter describing the membrane preparation and characterization work constitutes the major part of this project. Several variations of membrane layer deposition were tested in the effort to improve the hydrogen permeance without undue compromise in selectivity. One variation was in the material of the selective layer. Titanium, aluminum and boron oxides were tested in addition to silicon oxide. Another variation was in the geometry of deposition--one sided versus opposing reactants geometry. A third was in the gas phase reagent and a final variation was in the wall thickness and porous structure of the support tube. Silica turned out to be superior to the other three oxides, hence, the latter phases of the project were devoted exclusively to silica membranes.

The variations of deposition geometry, gaseous silica precursors, and support tubes were examined with the purpose of increasing the rate of the deposition reaction relative to that of diffusion (increasing Thiele modulus) thereby reducing the thickness of the deposit layer and increasing the hydrogen permeance. Of these variations decreasing the pore size of the support turned out to be the most beneficial. Unfortunately, porous Vycor tubing is not a commercial material and, in any case, it is not available in many pore sizes or different tube dimensions. Corning Inc. produces porous tubing (Code 7930) as an intermediate in the production of nonporous tubing (Code 7900) which has excellent thermal and mechanical properties--similar to those of quartz. Corning Inc. has supplied free of charge tubing of $5 \mathrm{~mm} \mathrm{ID}, 7 \mathrm{~mm}$ OD and $40 \AA$ mean pore diameter. They have also provided for a fee tubing of different diameter, wall thickness, and mean pore diameter custom-made for this project.

It turned out that glass blowing operations on tubing of small diameter or small wall thickness was difficult, resulting in frequent failures. Furthermore, the custom made tubing was very expensive, hence, the bulk of the work was carried out with the standard $5 \mathrm{~mm} \mathrm{ID} / 7 \mathrm{~mm} \mathrm{OD} / 40 \AA$ pore diameter tubing which was rugged and was obtained free of charge. 


\subsection{Materials}

\section{Porous Suppo-t Tubes}

Porous Vycor tubing of ten different types varying in dimensions and pore size were obtained from Corning Inc. Among those only three provided useful results in deposition experiments. The dimensions, pore sizes and permeances of these three types are given in Table 1.1 below.

Table 1.1 Properties of Vycor tubing (Corning Code 7930) used to prepare silica membranes.

\begin{tabular}{lllccc} 
Code & ID $(\mathrm{mm})$ & OD $(\mathrm{mm})$ & $\begin{array}{c}\text { Mean Pore } \\
\text { Diameter }(\AA)\end{array}$ & \multicolumn{2}{c}{$\begin{array}{c}\text { Permeance at } 450^{\circ} \mathrm{C}^{\mathrm{a}} \\
\left(\mathrm{cm}^{3}(\mathrm{STP}) / \mathrm{cm}^{2}-\mathrm{min}-\mathrm{atm}\right) \\
\mathrm{H}_{2}\end{array}$} \\
\hline $\mathrm{V} 1$ & 4.8 & 7 & 40 & $0.4-0.6$ & $0.14-0.22$ \\
$\mathrm{~V} 2$ & 5 & 5.66 & 25 & 1.05 & 0.31 \\
$\mathrm{~V} 3$ & 2 & 2.28 & 30 & $\mathrm{NA}$ & $\mathrm{NA}$ \\
\hline
\end{tabular}

a Variations were observed from batch to batch and among tubes of the same batch.

The tubes were prepared by the glassblower for connection to the CVD reactor as follows. A porous tube segment about $30 \mathrm{~cm}$ long was first heated at $100^{\circ} \mathrm{C}$ for one hour to drive off condensed and physically adsorbed water. After this preliminary heating, each side of the tube was heated gradually in a Bunsen flame to above $1000^{\circ} \mathrm{C}$ until the porosity collapsed in a section of a few centimeters at each end making that end completely nonporous, while the center section of $10-30 \mathrm{~cm}$ length remained porous. Finally short quartz sections $6 \mathrm{~mm}$ OD were welded to the nonporous Vycor sections at either end for ready connection to metal tubing using standard Swagelock fittings. 


\section{Chemicals}

$\mathrm{SiCl}_{4}(99.999 \%), \mathrm{SiBr}_{4}(99 \%), \mathrm{AlCl}_{3}(99.99 \%)$, and $\mathrm{TiCl}_{4}(99.9 \%)$ were purchased from Aldrich Chemical; $\mathrm{BCl}_{3}(99.999 \%)$ from Matheson Gas Products. The chlorosiloxanes $\mathrm{Cl}_{3} \mathrm{SiOSiCl}_{3}$ (hexachlorosiloxane) and $\mathrm{Cl}_{3} \mathrm{SiOSiCl}_{2} \mathrm{OSiCl}_{3}$ (octachlorodisiloxane) were purchased from Huls America, Inc. (formerly Petrarch Systems). High purity $\mathrm{N}_{2}, \mathrm{O}_{2}$, and $\mathrm{H}_{2}$ gases were supplied by Matheson Gas Products and further purified by Altech gas purifiers.

\subsection{Apparatus and Procedure Apparatus for Opposing Reactants Deposition}

The apparatus for membrane deposition, shown schematically in Figure 1.1 consists of a concentric tube reactor placed in an electrical furnace. The two reactant streams are generated by flowing two carrier $\mathrm{N}_{2}$ streams, typically at $50 \mathrm{~cm} 3 / \mathrm{min}$ each, through temperature controlled baths of the reactants, e.g. $\mathrm{H}_{2} \mathrm{O}$ bath and $\mathrm{SiCl}_{4}$ bath. For $\mathrm{Al}_{2} \mathrm{O}_{3}$ deposition, an $\mathrm{AlCl}_{3}$ sublimator was used in place of the chloride bubbler, while for $\mathrm{B}_{2} \mathrm{O}_{3}$ deposition a $\mathrm{BCl}_{3}$ bottle was used and the gaseous chloride stream was diluted with $\mathrm{N}_{2}$ to the desired concentration. The temperature of each bath is controlled to achieve the desired mol fraction of the respective reactant. The $\mathrm{H}_{2} \mathrm{O}-\mathrm{N}_{2}$ stream is passed through the reactor shell (annulus) while the $\mathrm{SiCl}_{4}-\mathrm{N}_{2}$ (or $\mathrm{AlCl}_{3}, \mathrm{TiCl}_{4}, \mathrm{BCl}_{3}$ ) stream is passed through the inner, support tube. One can interchange the path of the reactants and pass $\mathrm{H}_{2} \mathrm{O}$ through the bore of the support tube and $\mathrm{SiCl}_{4}$ through the annulus between the two concentric tubes. In either case the two reactants diffuse in opposite directions within the tube wall and react to form a solid oxide product, gradually plugging the pores in a narrow layer within the tube wall. The progress of the reaction is followed by periodically interrupting the flow of the reactants, flushing the inner tube and the annulus and measuring the permeance to various gases as described below. 


\section{Apparatus for One-Side Deposition}

The apparatus for one-sided deposition differs from that of the opposing reactants deposition only in the introduction of the reactants. Figure 1.2 shows introduction of both reactants through the bore of the support tube which was the arrangemert used. One can obviously introduce both reactants through the annulus, instead.

The reaction was in all cases conducted at atmospheric pressure both in the bore and in the annulus. For one-sided deposition, atmospheric pressure was maintained in the annulus by flow of pure nitrogen. The concentration of the chloride and water was varied between different series of experiments. Different temperature ranges were investigated for each reactant. Before reaction the support tube was heated at 100 and then $200^{\circ} \mathrm{C}$ under $\mathrm{N}_{2}$ flow to remove adsorbed water and then at $600^{\circ} \mathrm{C}$ under oxygen flow for several hours to remove any adsorbed organic impurities.

\section{Permeation Rate Measurements}

The permeation rates of $\mathrm{H}_{2}, \mathrm{~N}_{2}$ and occasionally other gases was measured by maintaining the membrane tube at a fixed pressure of the gas and measuring the flow to the annulus. For sufficiently high flows, the membrane tube was maintained at approximately 3 atmospheres and the flowrate to the annulus was measured using a bubble flowmeter operating at one atmosphere. Permeation rates below the limit measurable by the bubble flowmeter were measured by a sensitive pressure transducer (Baratron) connected to the computer. In this case the membrane tube was maintained at 2 atmospheres under flow while the outer annulus was evacuated and then isolated from vacuum. Using the transient pressure buildup measured by the pressure transducer the membrane permeance was then determined using the following equations (assuming ideal gas behavior):

$$
\begin{aligned}
& \mathrm{Q}\left[\mathrm{cm}^{3}(\mathrm{STP}) / \mathrm{cm}^{2}-\mathrm{atm}-\mathrm{min}\right]=\dot{\mathrm{V}} /(\mathrm{A} \Delta \mathrm{p}) \\
& \dot{\mathrm{V}}=\mathrm{K} \frac{\mathrm{dp}}{\mathrm{dt}}
\end{aligned}
$$


where $\mathrm{A}$ is the surface of the membrane, $\Delta \mathrm{p}$ the pressure difference across the membrane, $\mathrm{dp} / \mathrm{dt}$ the rate of pressure rise in the annulus, and $\mathrm{K}$ the calibration factor established between the pressure transducer and the bubble flowmeter.

\subsection{Results of Membrane Preparation}

\section{$\mathrm{SiO}_{2}$ Membranes}

During the first year of the contract, deposition experiments were conducted in the opposing reactants geometry. The results of these depositions are compiled in Table 1.2. At the beginning the temperature of deposition was varied in the range $200-800^{\circ} \mathrm{C}$. It was found that deposition is so slow at $200^{\circ} \mathrm{C}$ that the permeance of the tube remained unchanged after two hours of operation. Even at $600^{\circ} \mathrm{C}$ deposition for as many as 5 hours was not sufficient for complete pore plugging. Useful results were obtained at 700 and $800^{\circ} \mathrm{C}$. At $700^{\circ} \mathrm{C}$ essentially complete pore plugging was attained within 3 hours although continuing deposition after that time led to further reduction in the permeation rates, especially that of $\mathrm{N}_{2}$, evidently due to densification and to a possible slow thickening of the already formed layer. At $800^{\circ} \mathrm{C}$ the reaction is faster requiring about $1.5-2$ hours for romplete pore plugging. The time required for complete pore plugging by $\mathrm{SiCl}_{4}$ hydrolysis at 700 and $800^{\circ} \mathrm{C}$ is much longer than the $15-20$ minutes required in previous experiments using $\mathrm{SiH}_{4}$ oxidation at $450^{\circ} \mathrm{C}$.

Figure 1.3 shows a typical evolution of the $\mathrm{H}_{2}$ and $\mathrm{N}_{2}$ permeance with deposition time at 700 and $800^{\circ} \mathrm{C}$ (codes 3,4 in Table 1.2). Deposition at $800^{\circ} \mathrm{C}$ causes a more rapid and pronounced decline in the permeance of nitrogen and a higher $\mathrm{H}_{2}: \mathrm{N}_{2}$ selectivity $(5000$ vs 3500 at $700^{\circ} \mathrm{C}$ deposition). It must be noted that the measurement of very low nitrogen permeances entails significant error because of possible minute leaks in the system. As a result of this error, the true $\mathrm{H}_{2}: \mathrm{N}_{2}$ selectivities may in some cases be higher than the values listed in Table 1.2. 
As shown in Table 1.2, deposition of $\mathrm{SiO}_{2}$ is much faster in the one-sided geometry than in the opposing reactants geometry. Pore plugging for the V1 tubes $(40 \AA$ pore size) requires about $20-30$ minutes at $600^{\circ} \mathrm{C}$ and only 5 minutes or less at $750^{\circ} \mathrm{C}$. For he V2 tubes ( $25 \AA$ pore size) the time was one minute or less. It should be kept in mind that the reaction times listed in Table 1.2 are generally larger than the minimum times required for pore plugging. When the pore plugging time was less than two or three minutes, it was impractical to monitor the progress of deposition by inter.upting the reaction, purging, and measuring permeances. Hence, deposition was often continued beyond the point of pore plugging. In the one-sided geometry, continuing the deposition beyond pore plugging will result in slow thickening of the tube wall and decline of the membrane permeance. Although the effect of prolonging the reaction beyond pore plugging was not studied as such, it is expeci ' to be minor as long as the reaction time is no more than two or three times larger than th. pore plugging time.

The sharply difference in the times required for one-sided and opposing reactants deposition can be readily understood by the different level of reactant concentrations at the reaction region. In one-sided deposition the reaction region located just below the cylindrical surface bounding the tube wall. At that surface the reactant concentrations are near their free stream values (discounting external mass transfer). In opposing reactants deposition, the concentrations at the reaction region are small fractions of their boundary values because of the two diffusion gradients. As a result of the lower concentration, the reaction rate in the opposing reactant geometry is lower and, hence, the reaction region and the resulting deposit layer are thicker than in the one-sided geometry.

\section{$\mathrm{TiO}_{2}, \mathrm{Al}_{2} \mathrm{O}_{3}$, and $\mathrm{B}_{2} \mathrm{O}_{3}$ Membranes}

Table 1.3 shows the properties of membranes prepared by the deposition of $\mathrm{TiO}_{2}$, $\mathrm{Al}_{2} \mathrm{O}_{3}$, and $\mathrm{B}_{2} \mathrm{O}_{3}$ layers on porous Vycor tubes ( $\mathrm{V} 1$ type). It should be noticed that all $\mathrm{TiO}_{2}$ and $\mathrm{Al}_{2} \mathrm{O}_{3}$ membranes were prepared in the opposing reactants geometry. Several attempts made to prepare such membranes in the one-sided geometry were unsuccessful, 
with little or no change in the permeance of nitrogen--or hydrogen--taking place in several hours of deposition. Boron oxide membranes, however, could be prepared in both deposition geometries although most preparations were carried out in the opposing reactants geometry. The failure of deposition in the one-sided geometry to produce permselective layers will be discussed in a later section.

Concerning now the permeation properties of these various oxides, we note that the $\mathrm{TiO}_{2}$ membranes deposited at 450 and $600^{\circ} \mathrm{C}$ had hydrogen permeance only moderately lower than the $\mathrm{SiO}_{2}$ membranes prepared in the opposing reactants geometry. The $\mathrm{H}_{2}: \mathrm{N}_{2}$ permeance ratio of the $\mathrm{TiO}_{2}$ membranes was in the range 200-1500 depending on deposition time and temperature. Figure 1.4 shows the evolution of hydrogen and nitrogen permeances during the deposition reaction at 450 and $600^{\circ} \mathrm{C}$. The $\mathrm{TiO}_{2}$ membranes prepared at $800^{\circ} \mathrm{C}$ had much lower hydrogen permeance and selectivity than the $\mathrm{SiO}_{2}$ counterparts, indicating some structural difference in the $\mathrm{TiO}_{2}$ layer prepared at the higher temperatures. Below $600^{\circ} \mathrm{C}$ it is possible that $\mathrm{TiO}_{2}$ is stabilized by the surrounding $\mathrm{SiO}_{2}$ matrix (of the Vycor support) to either an amorphous phase or to anatase, whereas at higher temperatures transition to the denser rutile phase might take place. 1

Alumina membranes were prepared at 450,700 and $800^{\circ} \mathrm{C}$ in the opposing reactants geometry. The results are summarized in Table 1.3 and Figure 1.5. The hydrogen permeance of the $\mathrm{Al}_{2} \mathrm{O}_{3}$ membranes prepared at 700 and $800^{\circ} \mathrm{C}$ is substantially below that of the $\mathrm{SiO}_{2}$ membranes. The $\mathrm{H}_{2}: \mathrm{N}_{2}$ permeance ratio is below 400 , also well below that of the $\mathrm{SiO}_{2}$ membranes. Somewhat better $\mathrm{H}_{2}: \mathrm{N}_{2}$ ratio was obtained when the $\mathrm{Al}_{2} \mathrm{O}_{3}$ membranes prepared at 450 were subsequently annealed at $700^{\circ} \mathrm{C}$. However, the hydrogen permeance did not improve by this annealing.

Boron oxide layers could be deposited over a wide temperature range $\left.(50-800)^{\circ} \mathrm{C}\right)$. Deposition above $500^{\circ} \mathrm{C}$ produced membranes with low hydrogen permeance. Membranes prepared by deposition at temperatures between 100 and $450^{\circ} \mathrm{C}$ had hydrogen permeance as high as $0.2 \mathrm{~cm}^{3} / \mathrm{cm}^{2}-$ min-atm at $4500^{\circ} \mathrm{C}$ and $0.03 \mathrm{~cm}^{3} / \mathrm{cm}^{2}-$ min-atm at 150$)^{\prime \prime} \mathrm{C}$. At 150$)^{\circ} \mathrm{C}$ the 
hydrogen permeance of the $\mathrm{SiO}_{2}$ membranes was negligible. Unfortunately, the hydrogen permeance of the $\mathrm{B}_{2} \mathrm{C}$ nembran : declined significantly upon annealing above $500^{\circ} \mathrm{C}$. Figure 1.6 shows the evolution of $\mathrm{H}_{2}$ and $\mathrm{N}_{2}$ permeance at $150^{\circ} \mathrm{C}$ during deposition at the same temperature. Boron oxide membranes were subject to attack by water. Upon exposure to the laboratory atmosphere for several weeks, they developed boric acid whiskers and eventually cracked. Evidently, capillary condensation of water in the small Vycor pores and the smaller pores partly coated by $\mathrm{B}_{2} \mathrm{O}_{3}$ caused the conversion of $\mathrm{B}_{2} \mathrm{O}_{3}$ to $\mathrm{B}(\mathrm{OH})_{3}$ causing the mechanical failure. Figure 1.7 contains several Arrhenius plots of the hydrogen permeance of a $\mathrm{B}_{2} \mathrm{O}_{3}$ deposited at $150^{\circ} \mathrm{C}$, subsequently annealed and finally exposed to the laboratory atmosphere for 23 days.

\subsection{Membrane Characterization}

Membrane characterization was carried out by scanning electron microscopy (SEM) and electron microprobe analysis (EMA). SEM was conducted with a CamScan electron microscope operating at $20 \mathrm{kV}$ and with resolution of $500 \AA$. Both cylindrical surfaces (the side of the chloride flow) and cross sections of membrane tubes were examined. To examine a tube cross section the sample was cut, cast in epoxy resin, polished and coated with carbon or gold. In addition to SEM, tube cross sections were also examined by a Jeol 733 Superprobe electron microprobe by wavelength dispersive spectroscopy to obtain the elemental composition as a function of radial distance.

\section{Examination of Tube Cross Sections}

Tube cross sections after $\mathrm{CVD}$ of $\mathrm{SiO}_{2}, \mathrm{TiO}_{2}$ and $\mathrm{Al}_{2} \mathrm{O}_{3}$ were examined by SEM and EMA. Examination of $\mathrm{SiO}_{2}$ deposit layers turned out to be difficult when a focused electron beam was used because of sample burning. By defocusing the electron beam it was possible to obtain microprobe traces on $\mathrm{SiO}_{2}$ layers but at lower resolution. $\mathrm{Al}_{2} \mathrm{O}_{3}$ and $\mathrm{TiO}_{2}$ layers were easier to characterize. 
An SEM micrograph of an $\mathrm{Al}_{2} \mathrm{O}_{3}$ membrane obtained by opposing reactants deposition is shown on Figure 1.8. The darker region near the inner radius signifies the alumina layer, while the white line at the end of that region is an artifact due to polishing. Figure 1.9 shows the EMA irace of the alumina layer shown in Figure 1.8. The trace shows a gradually rising concentration of $\mathrm{Al}_{2} \mathrm{O}_{3}$ followed by a steep drop. The $\mathrm{Al}_{2} \mathrm{O}_{3}$ deposit covers a region of almost $90 \mu \mathrm{m}$ but the much narrower peak region is where Knudsen diffusion is completely disrupted and transport takes place by activated diffusion in the dense layir. during $\mathrm{Al}_{2} \mathrm{O}_{3}$ deposition in the opposing reactants geometry. Early during deposition the profile is monotonically declining, but later on its develops a maximum which sharpens until pore plugging when the reaction stops.

As stated earlier, the EMA of $\mathrm{SiO}_{2}$ layers required defocusing of the electron beam and hence gave relatively low resolution, about $5 \mu \mathrm{m}$, as opposed to the approximately $1 \mu \mathrm{m}$ resolution employed for the $\mathrm{Al}_{2} \mathrm{O}_{3}$ layers. Figure 1.11 shows the EMA trace of a $\mathrm{SiO}_{2}$ layer deposited in the opposing reactants geometry. This particular deposit layer was very wide and because of the lower resolution it does not show the sharp peak shown in the previous EMA scans. Figure 1.12 shows the EMA scan of a $\mathrm{SiO}_{2}$ layer deposited in one-sided geometry on a Vycor tube with $120 \mu \mathrm{m}$ mean pore diameter (V2, defined in Table 1.1) All other EMA traces shown here were obtained for deposition on support tubes with $40 \AA$ mean pore diameter (V1, defined in Table 1.1). As expected, the profile is monotonically declining and penetrates about $70 \mu \mathrm{m}$ from the chloride side. Complete disruption of Knudsen diffusion takes place only over a much thinner layer at the inner tube radius (zero distance from chloride flow). The thickness of this hydrogen permselective layer cannot be measured from the EMA scans because of the low resolution. EMA scans of two $\mathrm{SiO}_{2}$ membranes prepared by one-sided deposition on Vycor tubes with $40 \AA$ mean pore diameter are shown in Figure 1.13. The main difference between the traces of Figures 12 and 13 is the much shallower deposit penetration in the latter. Although different 
reactant concentrations were used in these depositions, the difference in the depth of penetration is primari' $y$ due to the different pore size of the support tubes.

\section{Examination of Cylindrical Surface (side of reactants flow)}

The inside cylindrical surface exposed to the $\mathrm{SiCl}_{4}, \mathrm{H}_{2} \mathrm{O}$ reactants was examined by SEM. Figure 1.14a shows a micrograph of a Vycor tube before deposition. Although these tubes have $40 \AA$ mean pore diameter as determined by nitrogen adsorption, the pore mouths seen on the micrograph are much larger, perhaps in the range 200-300 $\AA$. It appears that in the leaching process used to prepare the Vycor tubes, larger pores are developed in a thin skin near the external surface. Transmission electron micrographs (not shown here) verify that the pores in the bulk of the Vycor have mean pore diameter of about $40 \AA$. Figure $1.14 \mathrm{~b}$ shows a micrograph of a surface after deposition with low concentration of $\mathrm{SiCl}_{4}$. Comparison of Figures $1.14 \mathrm{a}$ and $1.14 \mathrm{~b}$ reveals that deposition does not change significantly the texture of the surface and suggests that the deposit layer does not extend outside of the porous matrix. To investigate the evolution of the deposit layer when deposition is continued beyond the time of pore plugging, we prolonged deposition under the conditions of Figure $1.14 \mathrm{~b}$, for 15 and 35 additional minutes after pore plugging (30 and 50 minutes of total deposition time). Examination of the surface by SEM (Figure 1.15) indicates that after pore plugging the deposit continues to grow outside of the pore matrix.

The influence of the $\mathrm{SiCl}_{4}$ reactant concentration on the morphology of the deposit is revealed by comparison of Figure 1.16 with Figure 1.14b. Figure 1.16 shows the micrograph of the surface after deposition with $8.7 \% \mathrm{SiCl}_{4}, 13 \% \mathrm{H}_{2} \mathrm{O}$ for 30 minutes (immediately after pore plugging). With both reactants at high concentration, a pronounced surface roughness is observed indicating the adherence on the surface of particles which are too large to enter into the pores. Pore plurging still takes place by penetration and heterogeneous reaction of $\mathrm{SiCl}_{4}$, and possibly some oligomers, on the internal pore surface area. Since the monomer concentraticsii is reduced by the homogeneous reactions, 
the time for pore plugging is increased to 30 minutes as compared to the 15 minutes that were required for the conditions of Figure 1.14b.

\subsection{Discusion}

The mechanism of $\mathrm{SiO}_{2}$ deposition by $\mathrm{SiCl}_{4}$ hydrolysis has been discussed in two recent publications deriving from this project. ${ }^{1.2}$ The reaction takes place homogeneously and heterogeneously. In opposing reactants deposition, the reaction region is wholly inside the porous matrix of the support where because of the low reactant concentrations and the large surface area per unit volume deposition occurs by heterogeneous reaction. Any dimers or oligomers generated homogeneously would also react with the surface before diffusing outside. In one-sided deposition, the maximum concentration of reactants is in the bore (or in the annulus) so that homogeneous reaction as well as heterogeneous reaction take place. If the homogeneous reaction is too fast, the reactant $\mathrm{SiCl}_{4}$ becomes largely depleted before it can diffuse inside the pores. This seems to be the case with the $\mathrm{TiCl}_{4}$ $\mathrm{H}_{2} \mathrm{O}$ and $\mathrm{AlCl}_{3}-\mathrm{H}_{2} \mathrm{O}$ reactions where deposition fails to plug the pores of the support. In the case of $\mathrm{SiO}_{2}$ deposition under the temperatures and reactant concentrati sus employed the homogeneous $\mathrm{SiCl}_{4}-\mathrm{H}_{2} \mathrm{O}$ reaction is not fast enough to exhaust the reactants and prevent pore plugging. However, at $\mathrm{SiCl}_{4}$ pressures above about $10 \%$ the particles generated by the homogeneous reaction adhere to the bore surface and can cause thermal stresses upon cooling and reheating as will be discussed in the following chapter.

While penetration of the reactants into the pore matrix is essential in one-sided deposition, the depth of penetration must be kept as small as possible to obtain a thin membrane and high hydrogen permeance. This depth of penetration is approximately given by

$$
\delta=\left(\frac{D_{0}}{\mathrm{k}[\mathrm{OH}]_{0} \mathrm{~S}}\right)^{1 / 2}
$$

where $\mathrm{k}$ is the rate constant for the reaction of $\mathrm{SiCl}_{4}$ with surface $-\mathrm{OH}$ groups, $[\mathrm{OH}]_{0}$ is the initial surface concentration of - $\mathrm{OH}$ groups, $\mathrm{S}$ is the pore surface area per unit volume, and 
$D_{0}$ is the initial (Knudsen) effective diffusivity. For a given support material the penetration $\delta$ may be decreased by increasing the rate constant $\mathrm{k}$ and/or decreasing the diffusion coefficient $D_{0}$. The reaction rate may be increased by increasing the temperature. Raising the temperature, however, has the undesirable effects of eliminating surface $-\mathrm{OH}$ groups (decreasing $[\mathrm{OH}]_{\mathrm{o}}$ ), intensifying the homogeneous reaction, and above $900^{\circ} \mathrm{C}$, collapsing the porous structure. It appears that the optimal deposition temperature by the $\mathrm{SiCl}_{4}-\mathrm{H}_{2} \mathrm{O}$ reaction is about $750^{\circ} \mathrm{C}$.

In section 1.2 we listed two silicon reagents, in addition to $\mathrm{SiCl}_{4}$, which were used in a limited number of preparation experiments. These reagents, $\mathrm{Cl}_{3} \mathrm{SiOSiCl}_{3}$ (hexachlorosiloxane) and $\mathrm{Cl}_{3} \mathrm{SiOSiCl}_{2} \mathrm{OSiCl}_{3}$ (octachlorodisiloxane) have higher $\mathrm{k}$ (more $\mathrm{Cl}$ groups) and lower $D_{0}$ (higher molecular weight) than $\mathrm{SiCl}_{4}$, and as a result they should have smaller penetration depth $\delta$. Only slight improvement of hydrogen permeance was obtained with these reagents, but there is some scope for additional improvement.

A more significant variation that was briefly explored was the use of Vycor support tubes of smaller pore diameter. Decreasing the pore diameter increases $[\mathrm{OH}]_{\mathrm{o}}$ (more surface area) and decreases $D_{0}$ resulting, again, at a smaller penetration depth $\delta$. Because of the lower $D_{0}$, the thickness of the tube wall should also be decreased, otherwise the larger resistance of the untreated support tube would limit the advantage of a thinner deposit layer.

\section{Literature Cited}

1. Tsapatsis, M., Kim, S., Nam, S-W, and Gavalas, G. R., I\&EC Research 30, 2152 (1991).

2. Tsapatsis, M., and Gavalas, G. R., AIChE J. 38, 847 (1992). 


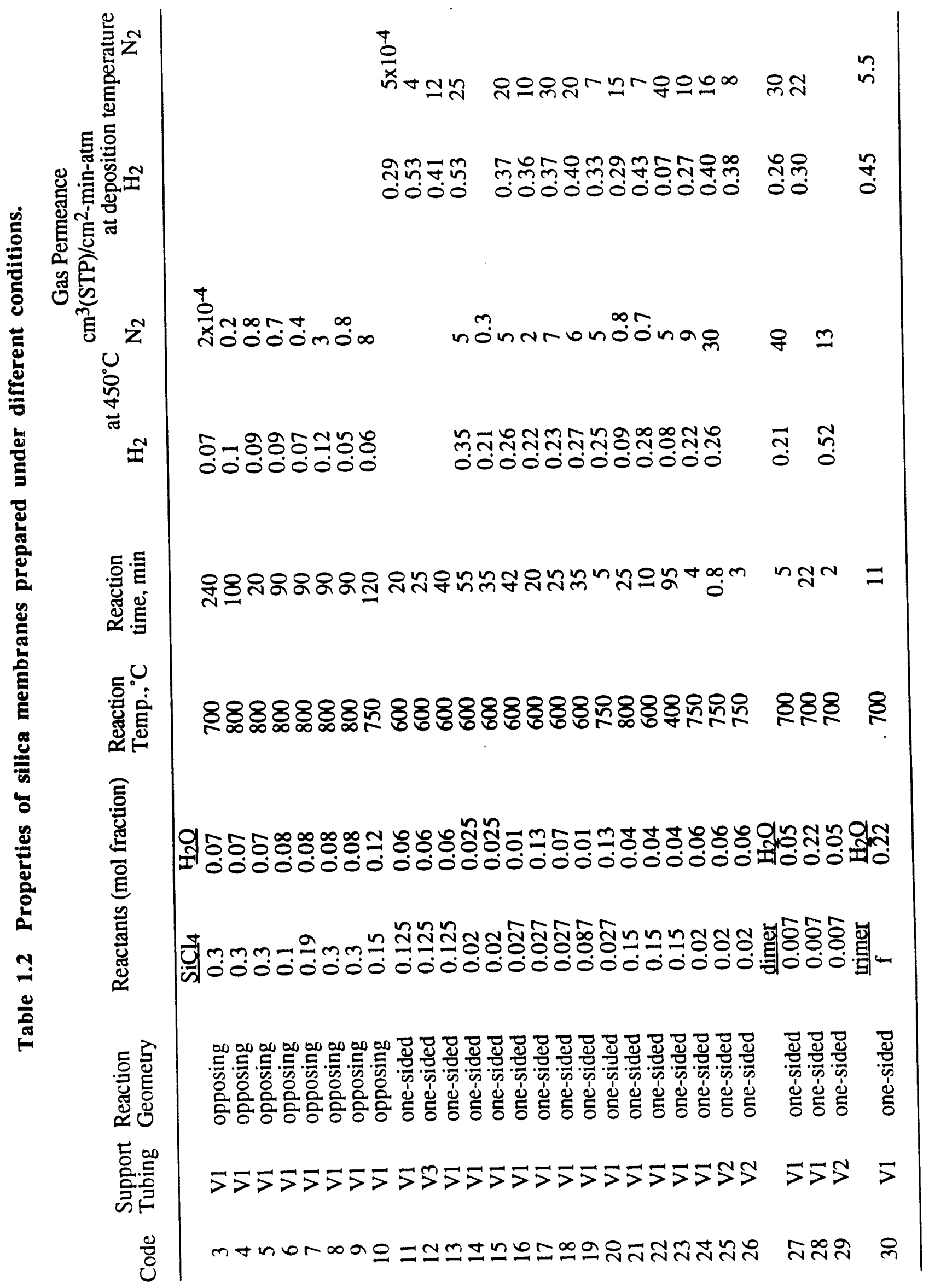




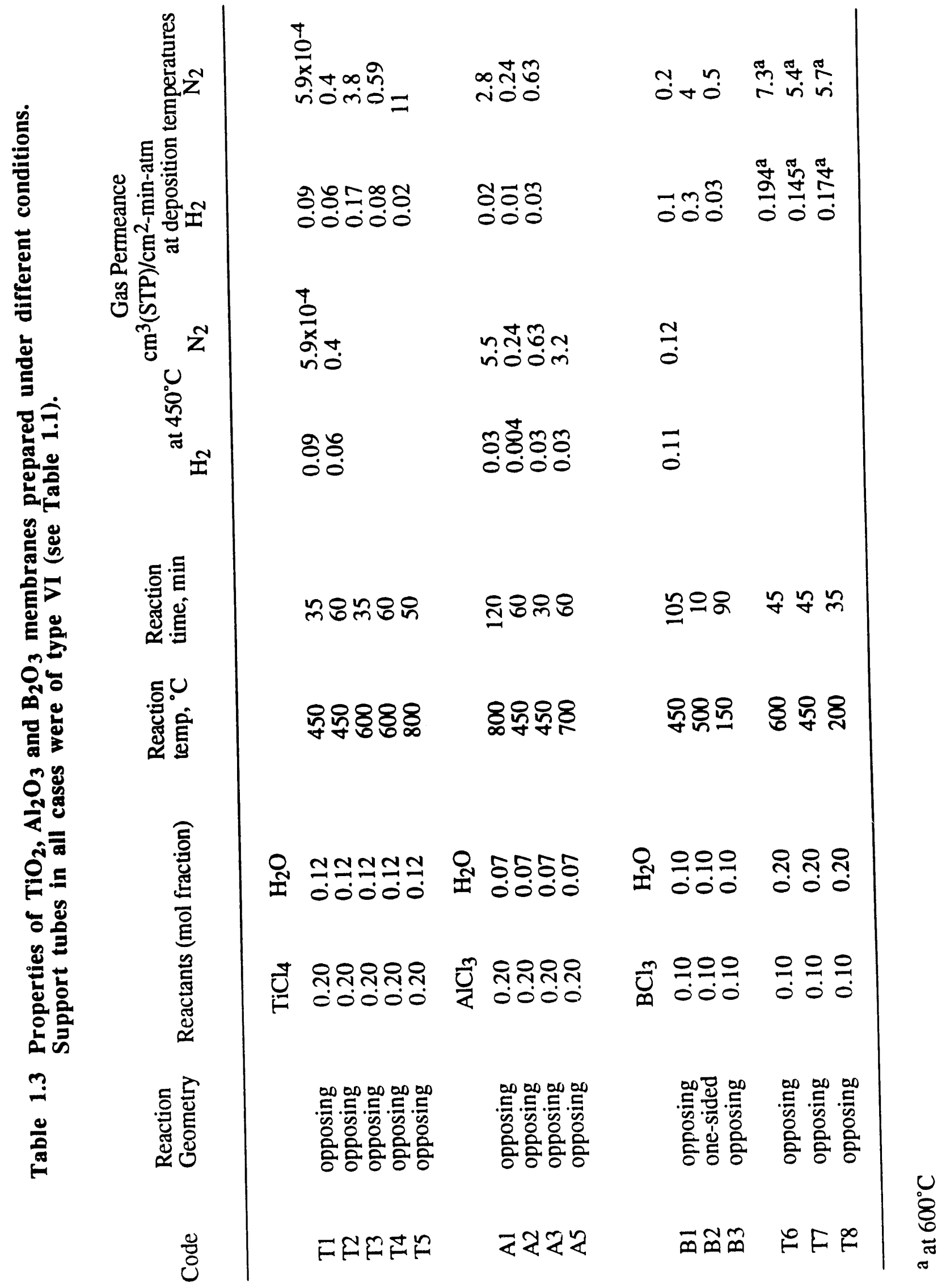




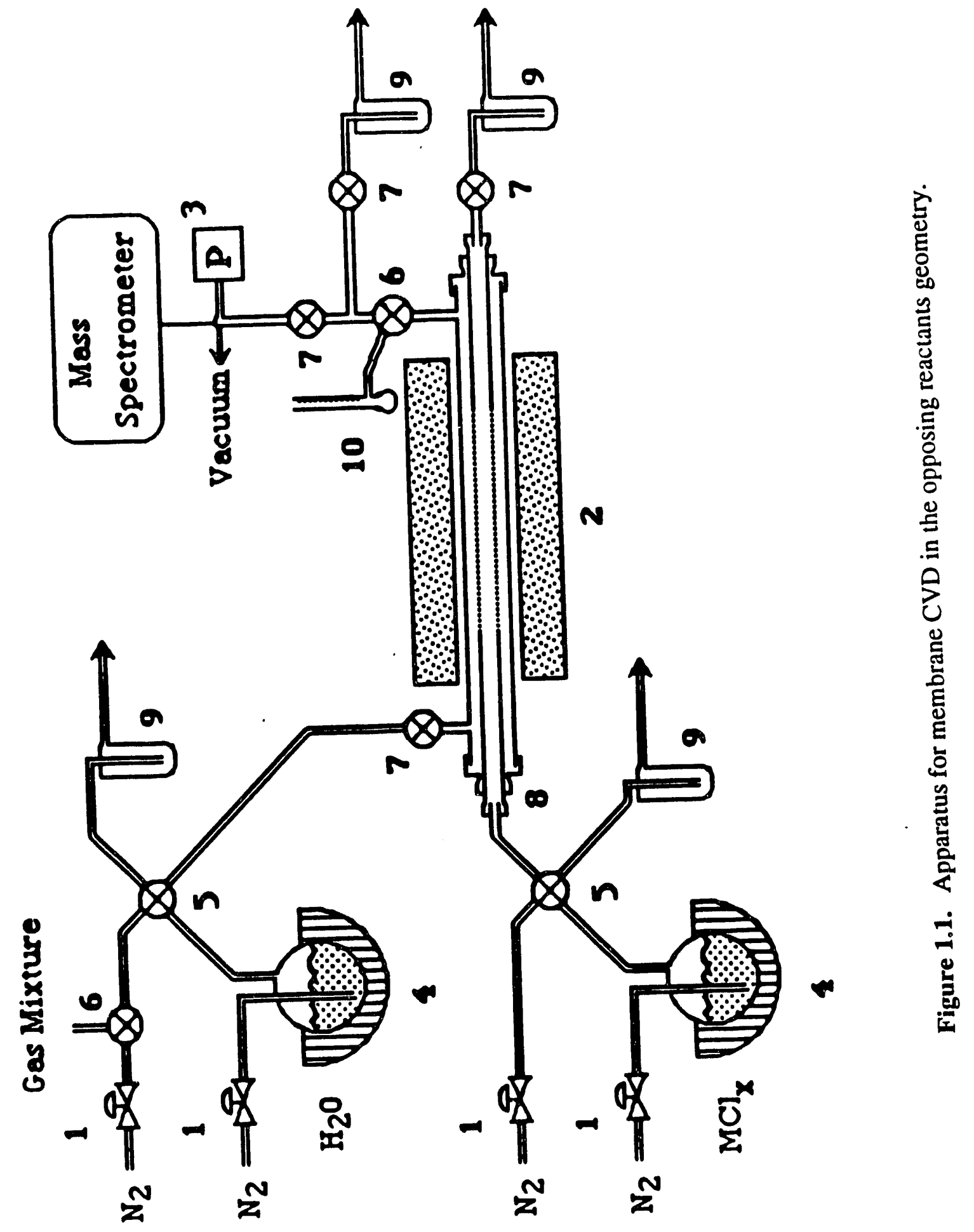




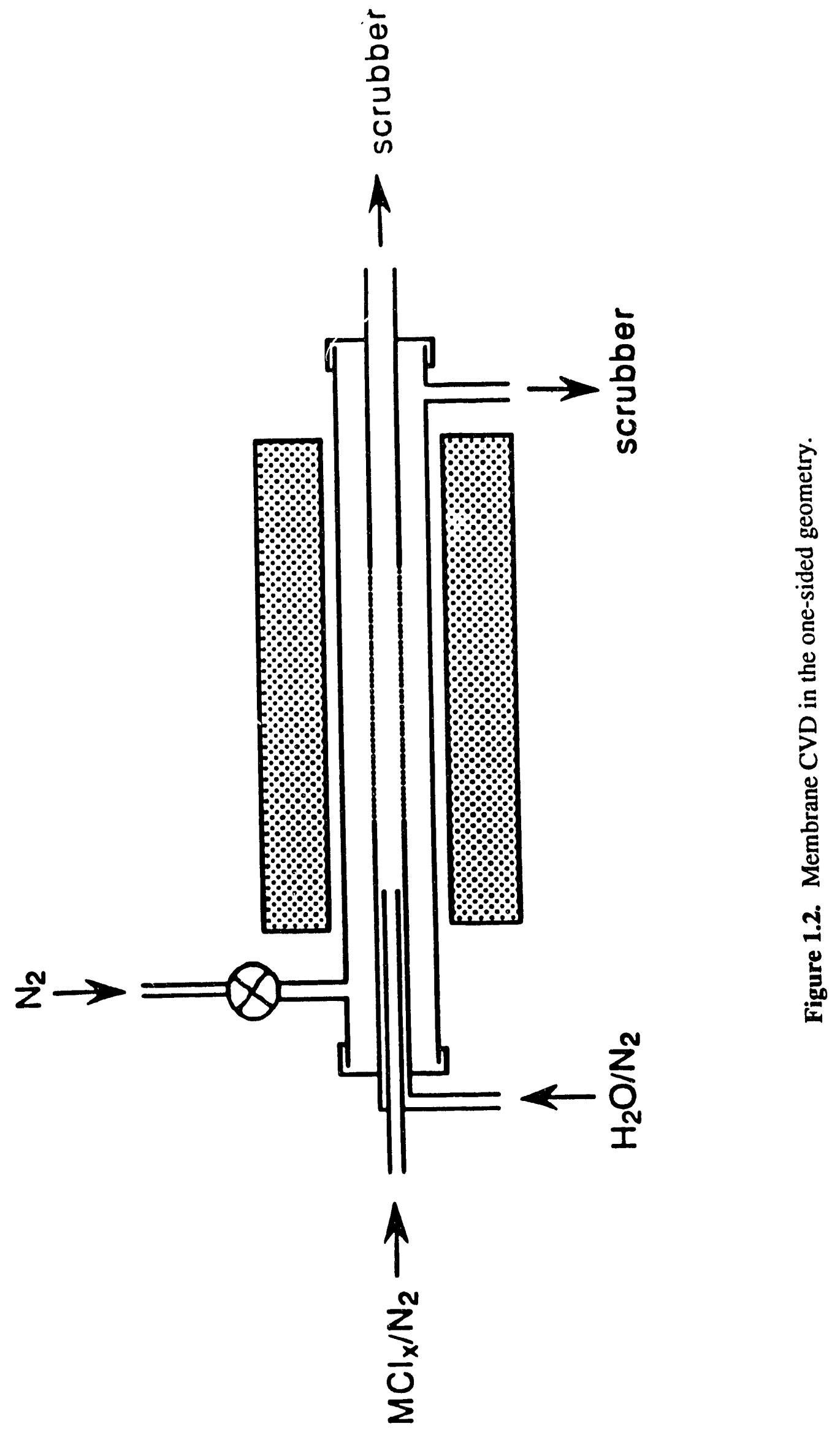



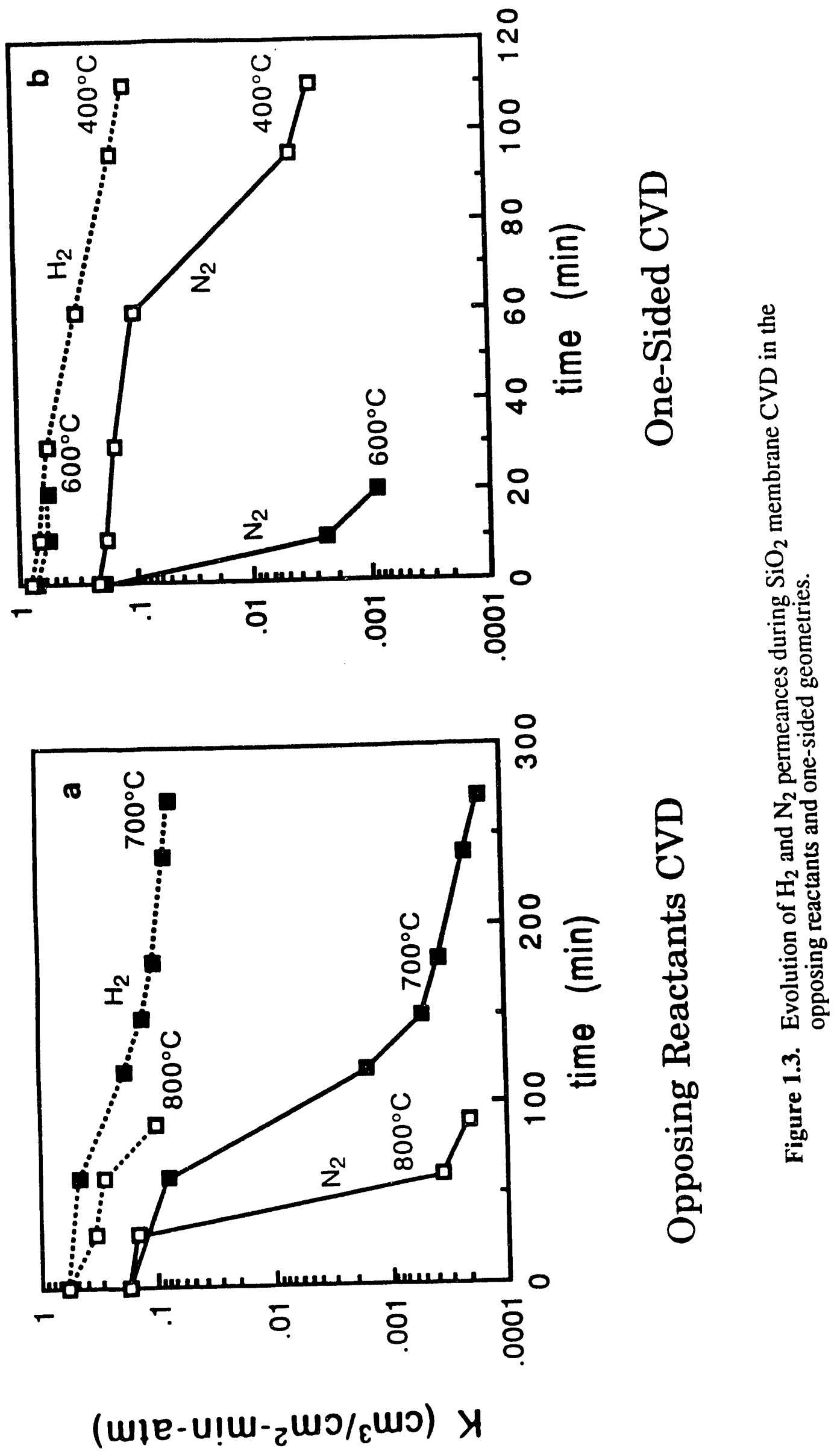


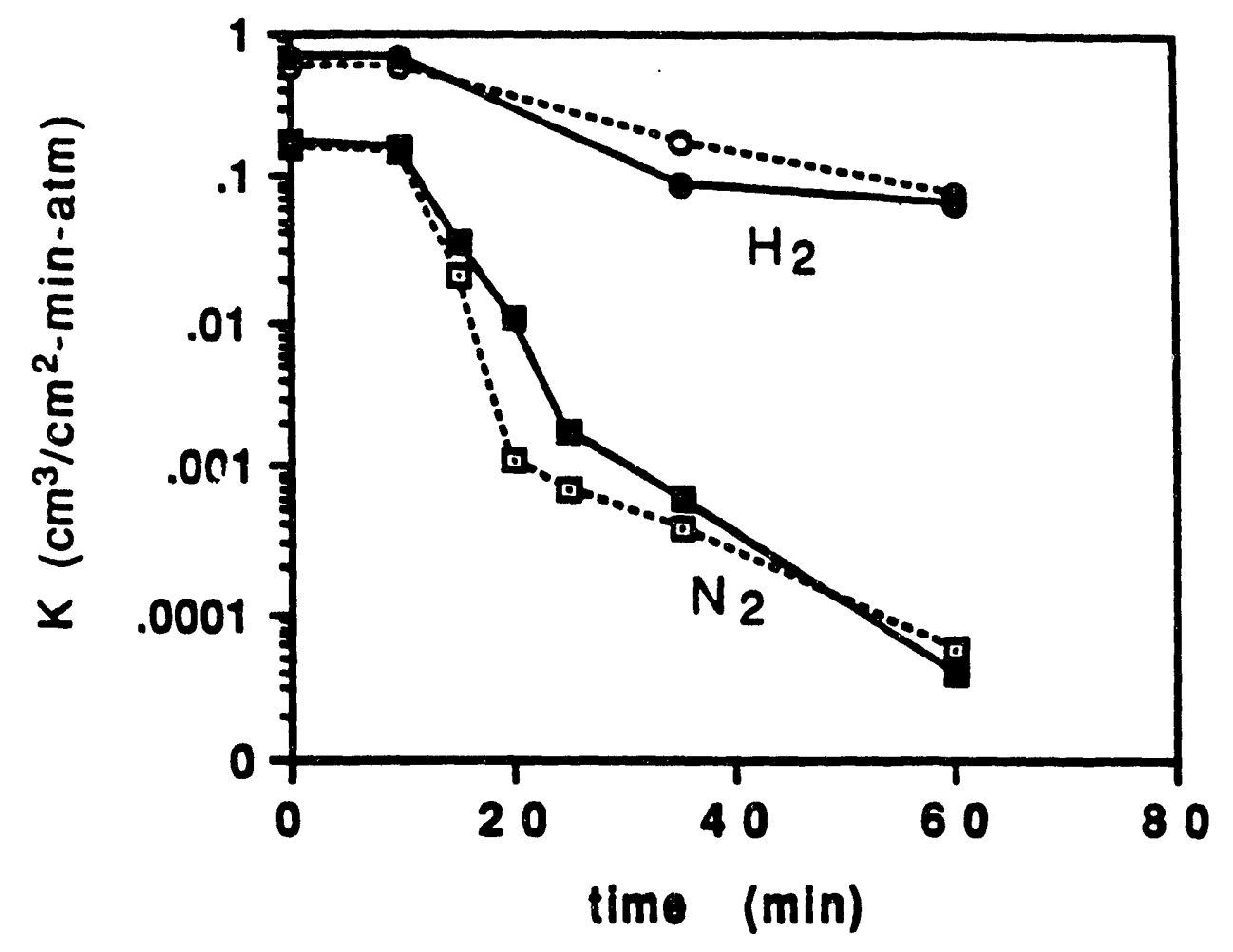

Figure 1.4. Nitrogen $(\square, \nabla)$ and hydrogen $(0, \bullet)$ permeation coefficients of $\mathrm{TiO}_{2}$ membranes during deposition at $450^{\circ} \mathrm{C}(-)$ and $\left.600^{\circ} \mathrm{C}\right)(--)$. Reactants: $20 \% \mathrm{TiCl}_{4}-\mathrm{N}_{2}$ and $12 \% \mathrm{H}_{2} \mathrm{O}-\mathrm{N}_{2}$. 


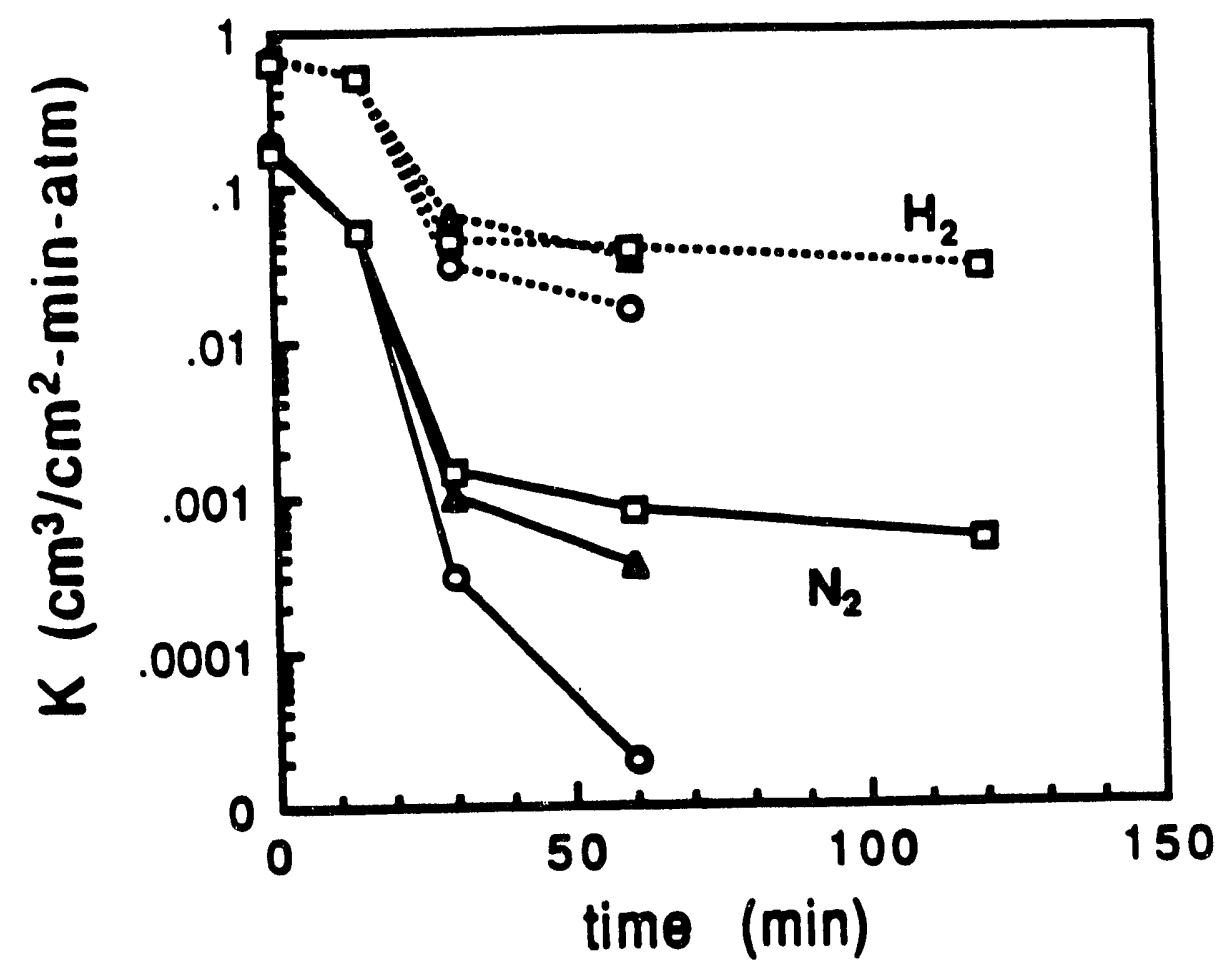

Figure 1.5. Permeation rate coefficients measured at deposition temperatures for hydrogen (-.) and nitrogen (-) versus time of $\mathrm{AlCl}_{3}+\mathrm{H}_{2} \mathrm{O}$ reaction at $450^{\circ} \mathrm{C}(\mathrm{O}), 700^{\circ} \mathrm{C}(\Delta), 800^{\circ} \mathrm{C}(\square)$. Reactant streams were $7 \% \mathrm{H}_{2} \mathrm{O}$ $\mathrm{N}_{2}$ and $20 \% \mathrm{AlCl}_{3}-\mathrm{N}_{2}$ in opposing reactants geometry. 


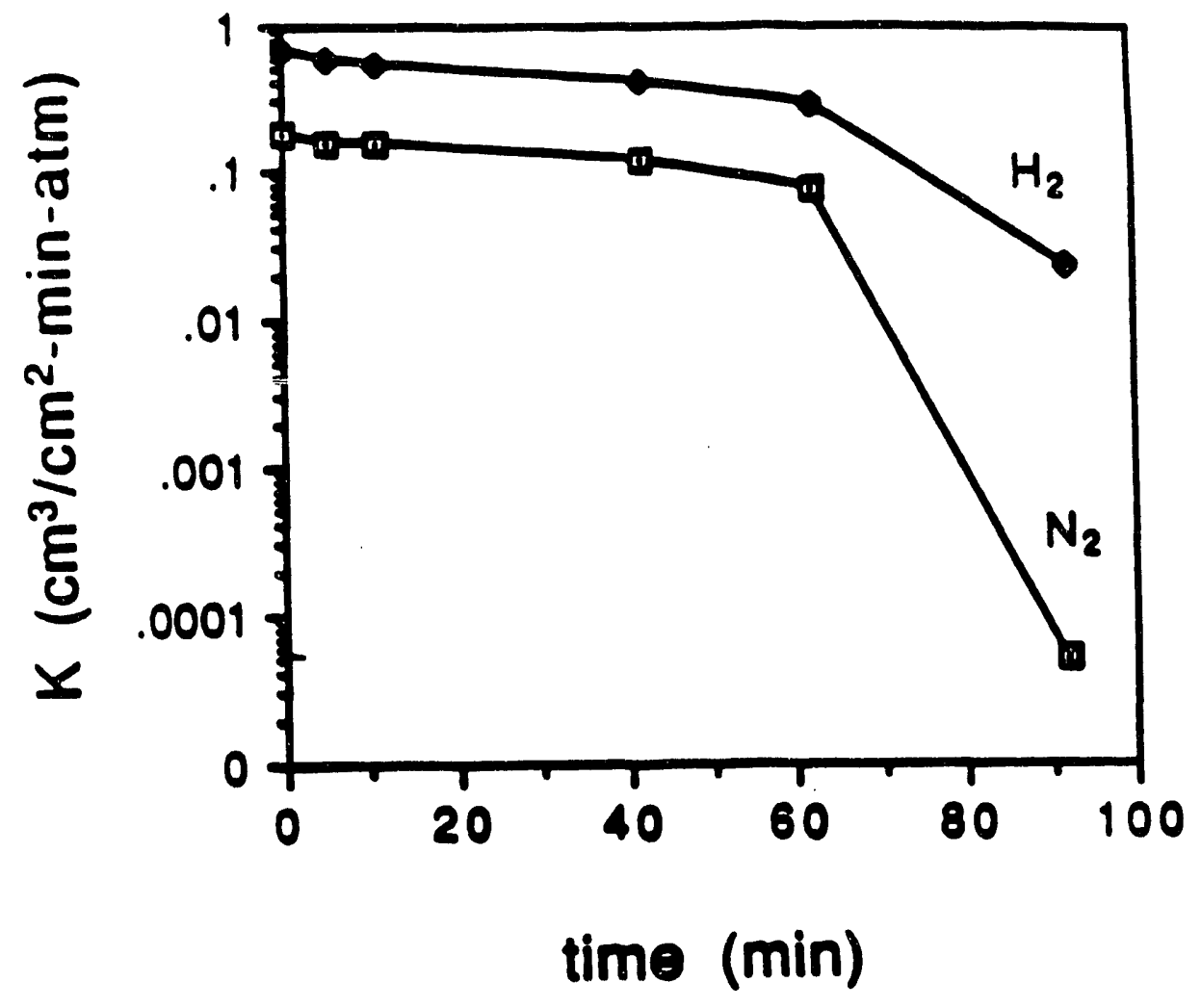

Figure 1.6. Hydrogen and nitrogen permeation coefficients at $150^{\circ} \mathrm{C}$ during deposition of $\mathrm{B}_{2} \mathrm{O}_{3}$ at $150^{\circ} \mathrm{C}$ in opposing reactants geometry. Reactant streams: $10 \% \mathrm{BCl}_{3}-\mathrm{N}_{2}$ and $10 \% \mathrm{H}_{2} \mathrm{O}-\mathrm{O}_{2}$. 


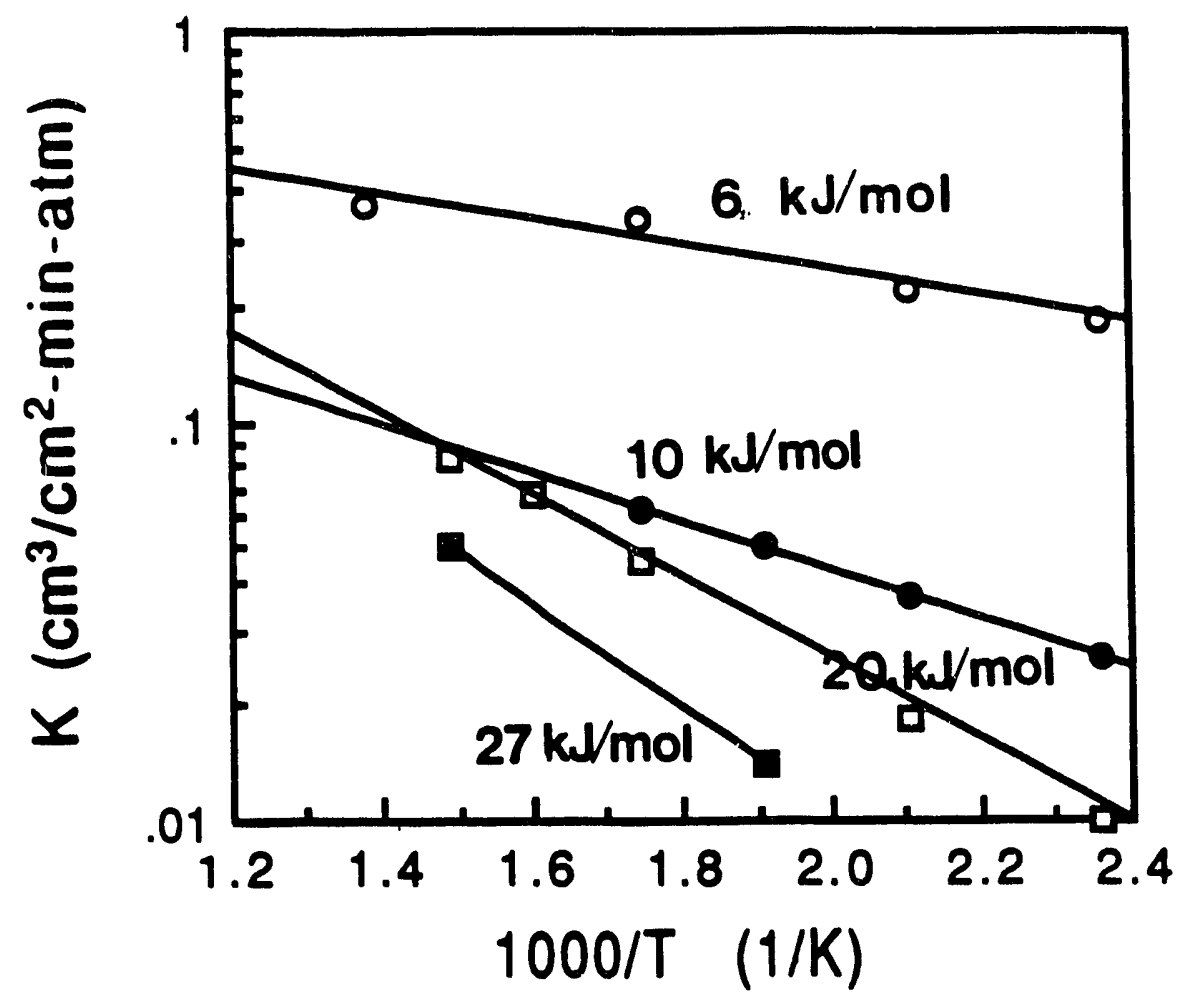

Figure 1.7. Hydrogen permeability coefficients of a $\mathrm{B}_{2} \mathrm{O}_{3}$ membrane deposited at $150^{\circ} \mathrm{C}$ and annealed under dry $\mathrm{N}_{2}$ at $400^{\circ} \mathrm{C}$ overnight $(\square), 300^{\circ} \mathrm{C}$ (a) overnight, $150^{\circ} \mathrm{C}$ overnight $(\Theta)$ and exposed to laboratory air at room temperature for 23 days (0). Calculated activation energies are given on the figure. 


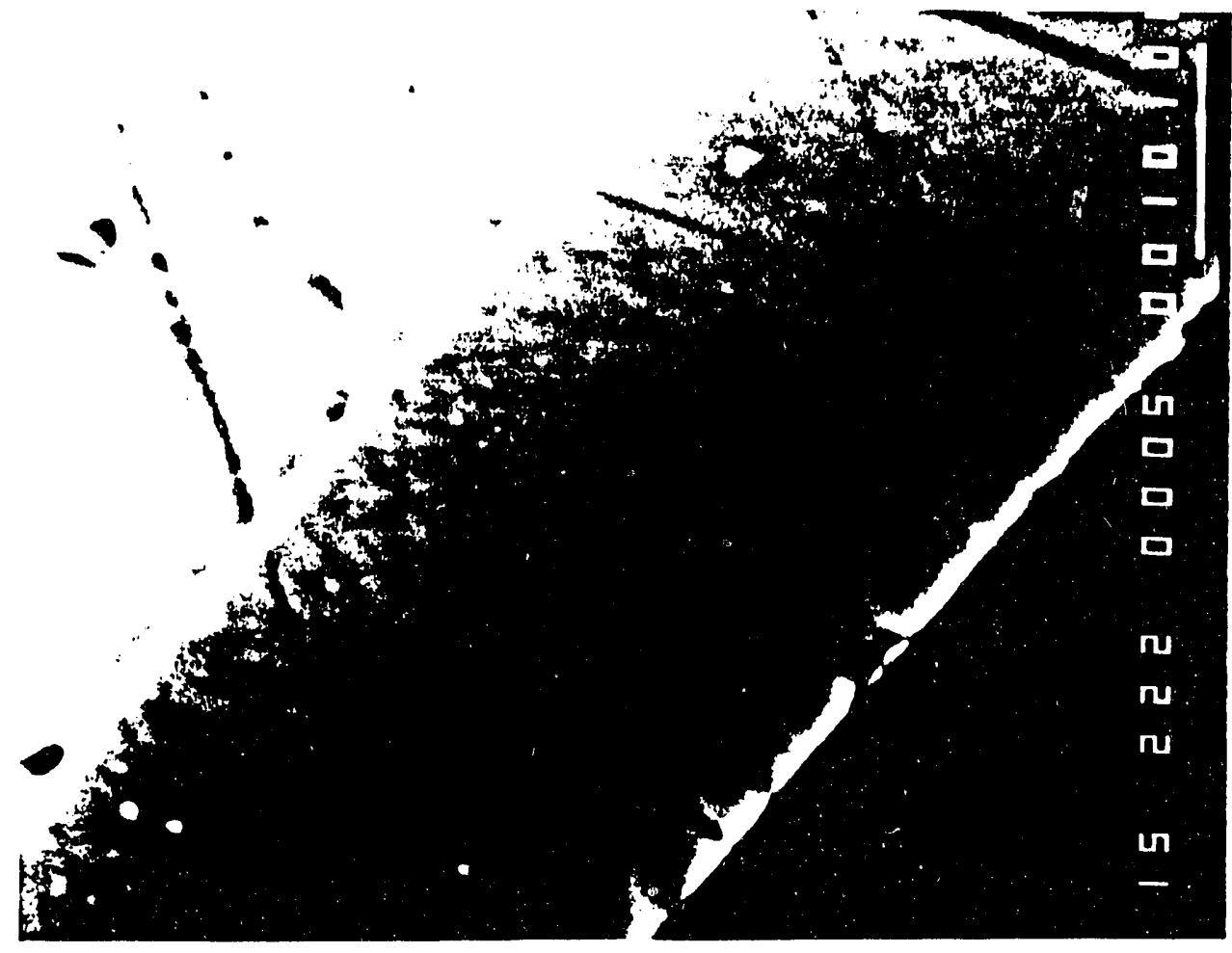

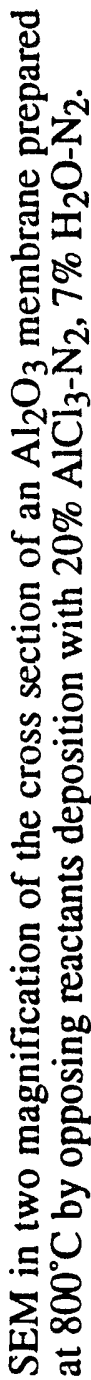

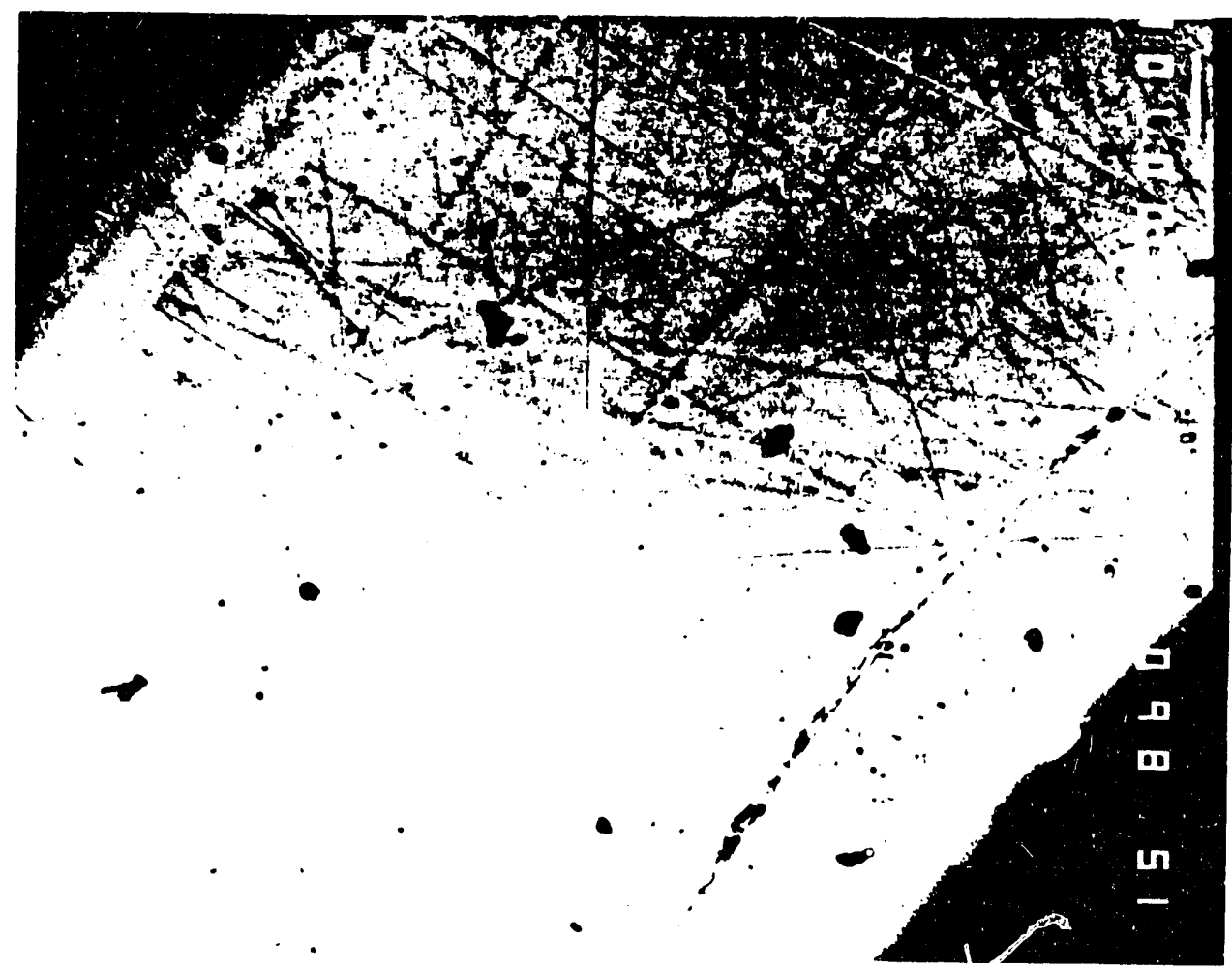

赵 


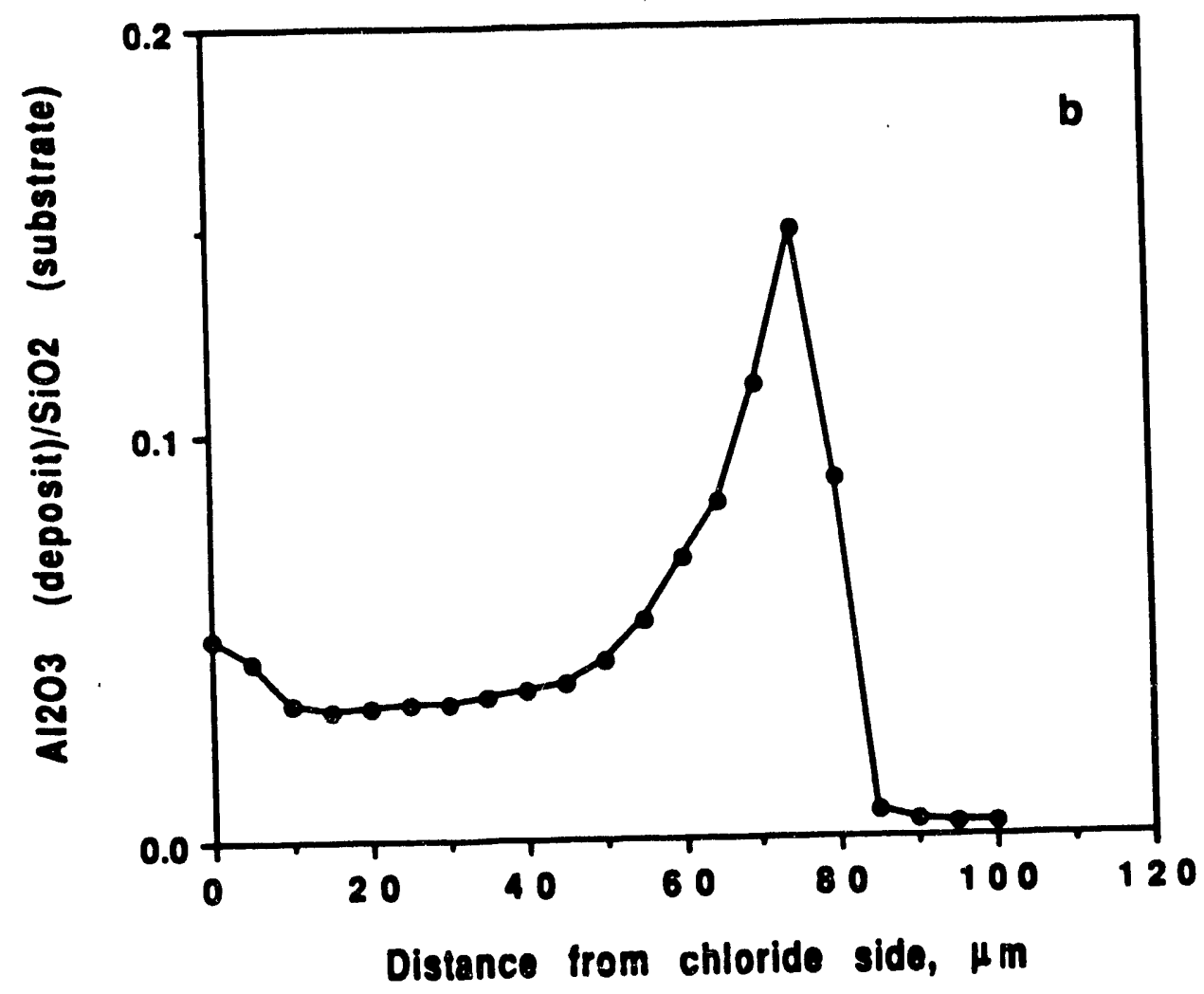

Figure 1.9. EMA trace of the $\mathrm{Al}_{2} \mathrm{O}_{3}$ layer shown in Figure 1.8. 


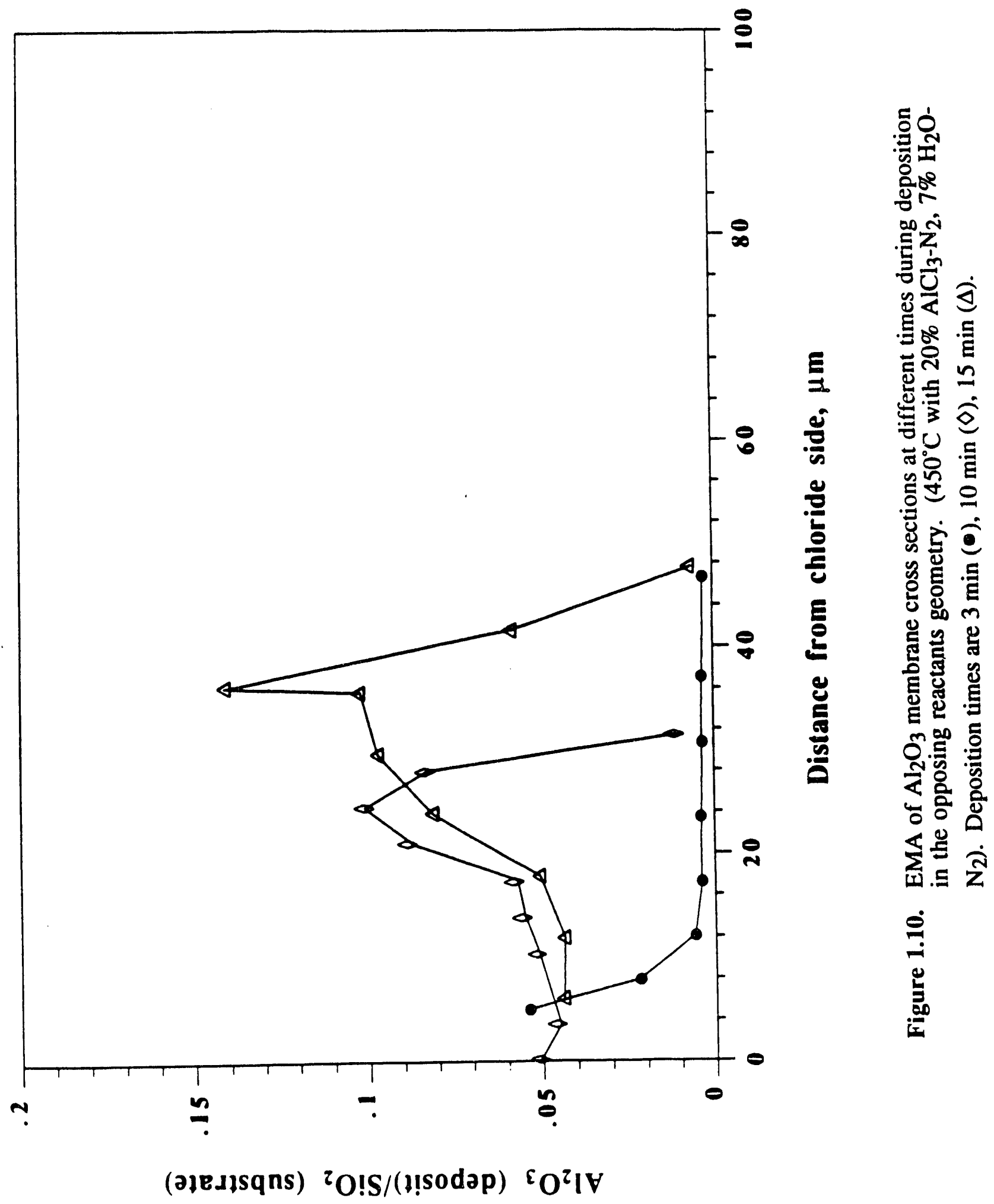




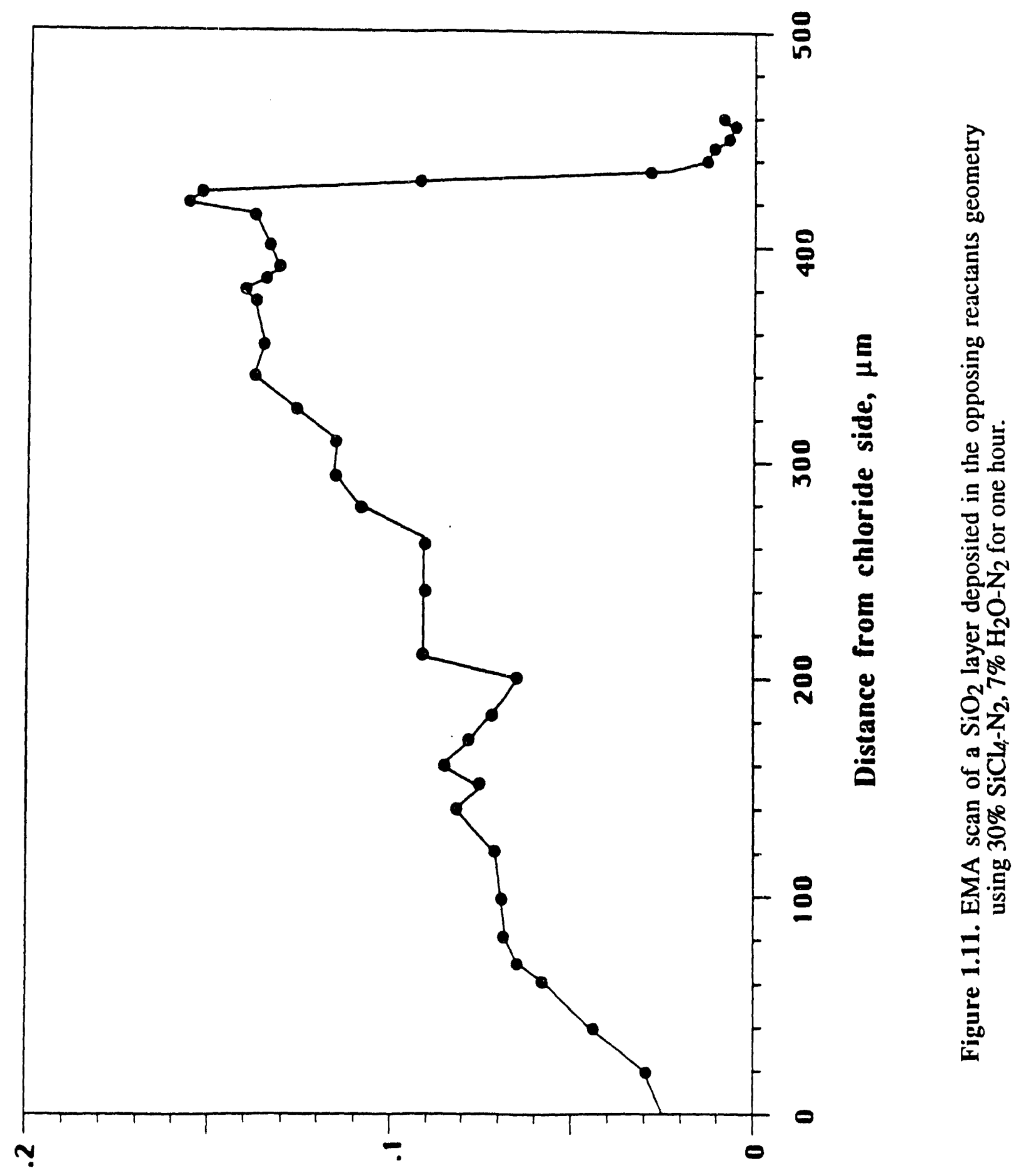

(วןedısqns) $20 ! S /(1 ! s o d \partial p) ~ ₹ O ! S$ 


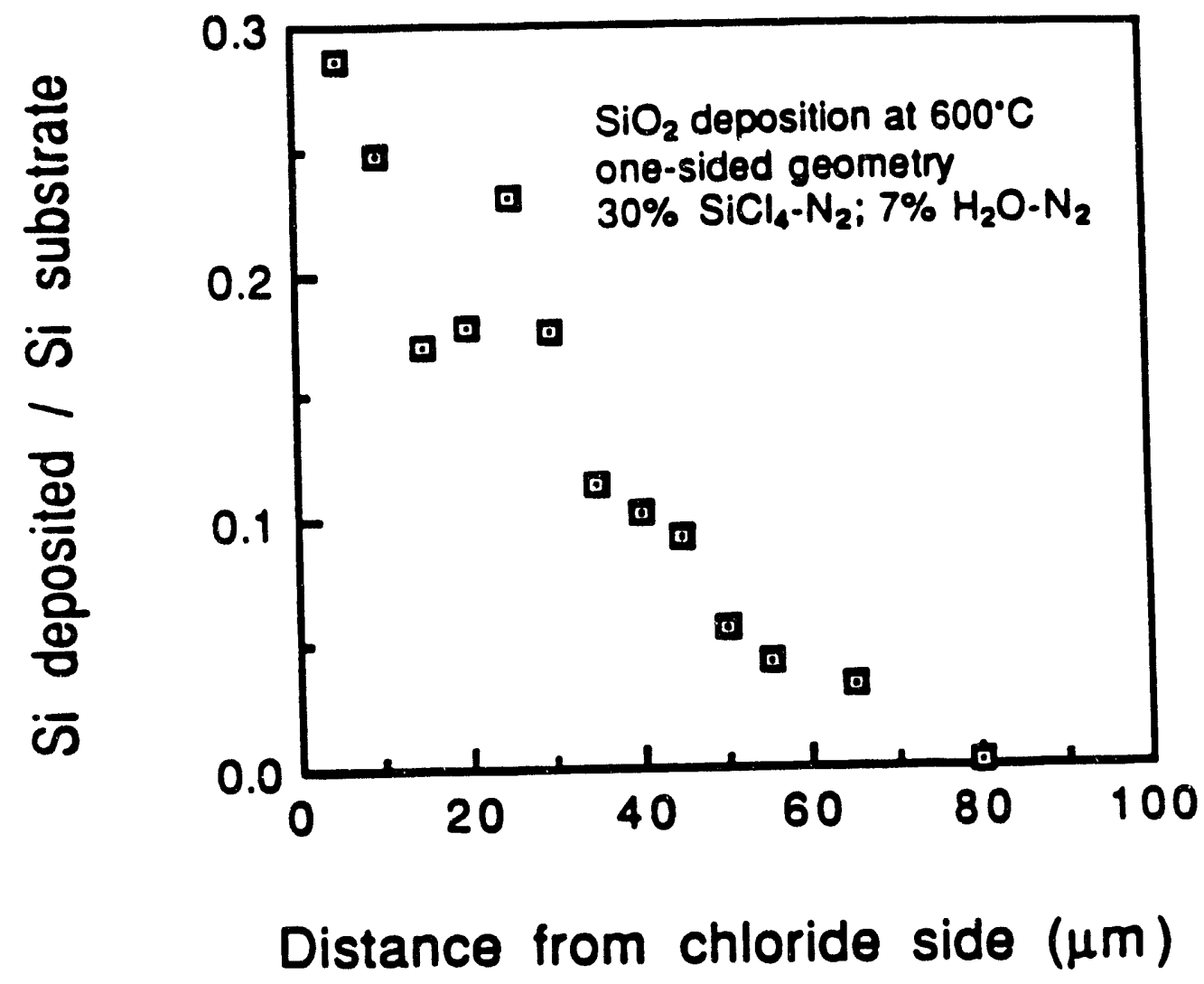

Figure 1.12. EMA of a $\mathrm{SiO}_{2}$ layer deposited in one-sided geometry on a Vycor tube of $120 \AA$ mean pore diameter. 


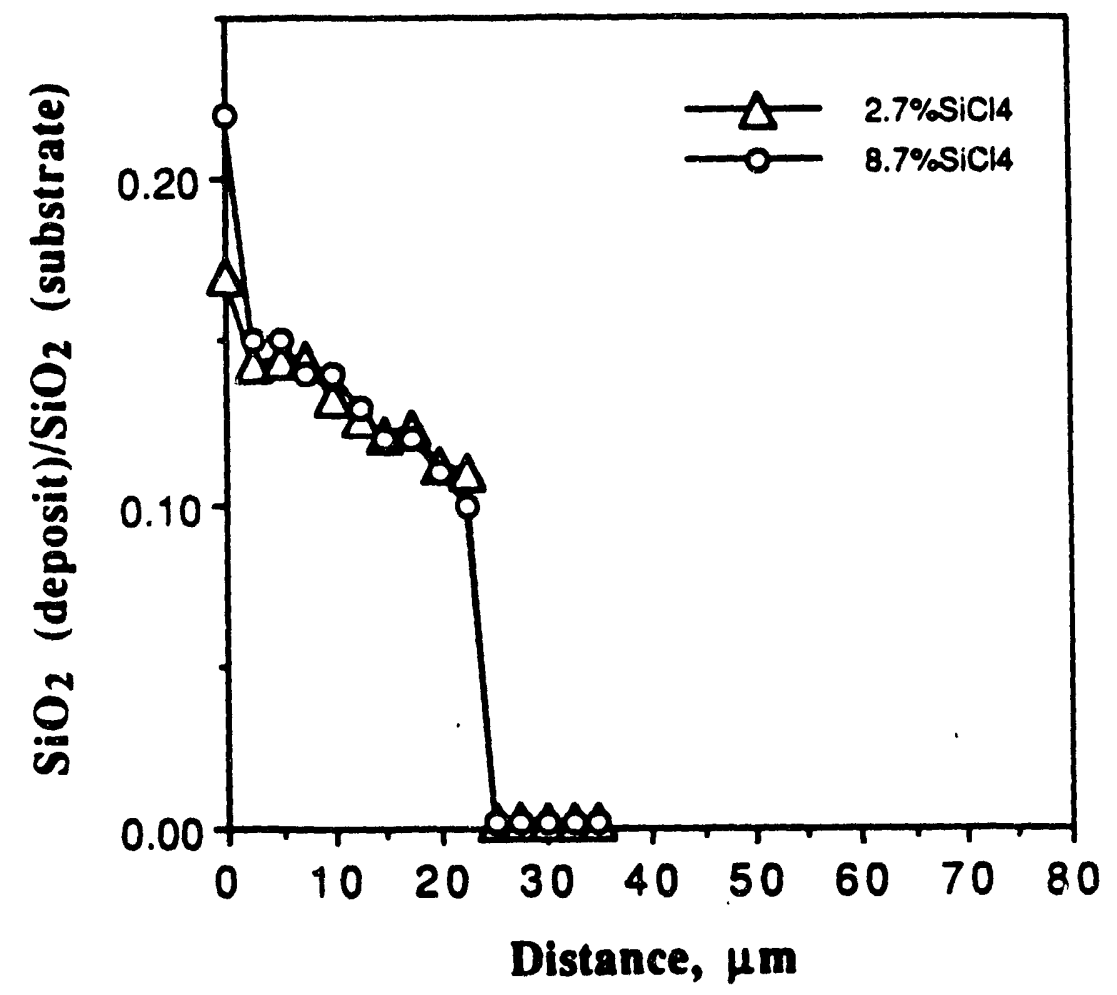

Figure 1.13. EMA of silica membranes deposited at $600^{\circ} \mathrm{C}$ in $\mathrm{OSG}$ with $13 \% \mathrm{H}_{2} \mathrm{O}$. 

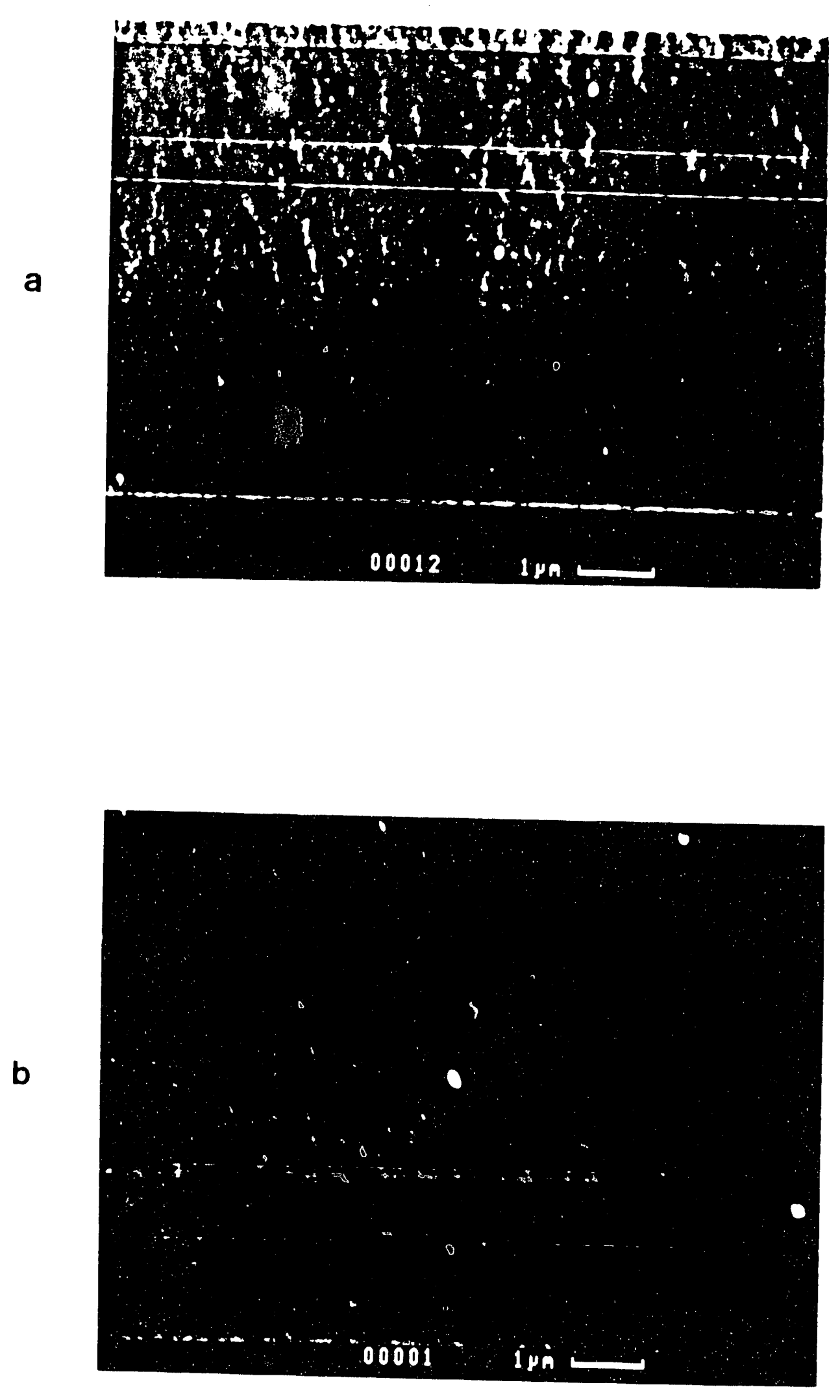

Figure 1.14. SEM micrographs of the bore surface of a Vycor tube (a) hefore deposition (h) after deposition in one-sided geometry with $2.7 \% \mathrm{SiCl}_{4}$ and $\left.13 \% \mathrm{H}_{2} \mathrm{O}\right)$ at $6(0) 1 \mathrm{C}$ for 15 minutes. 

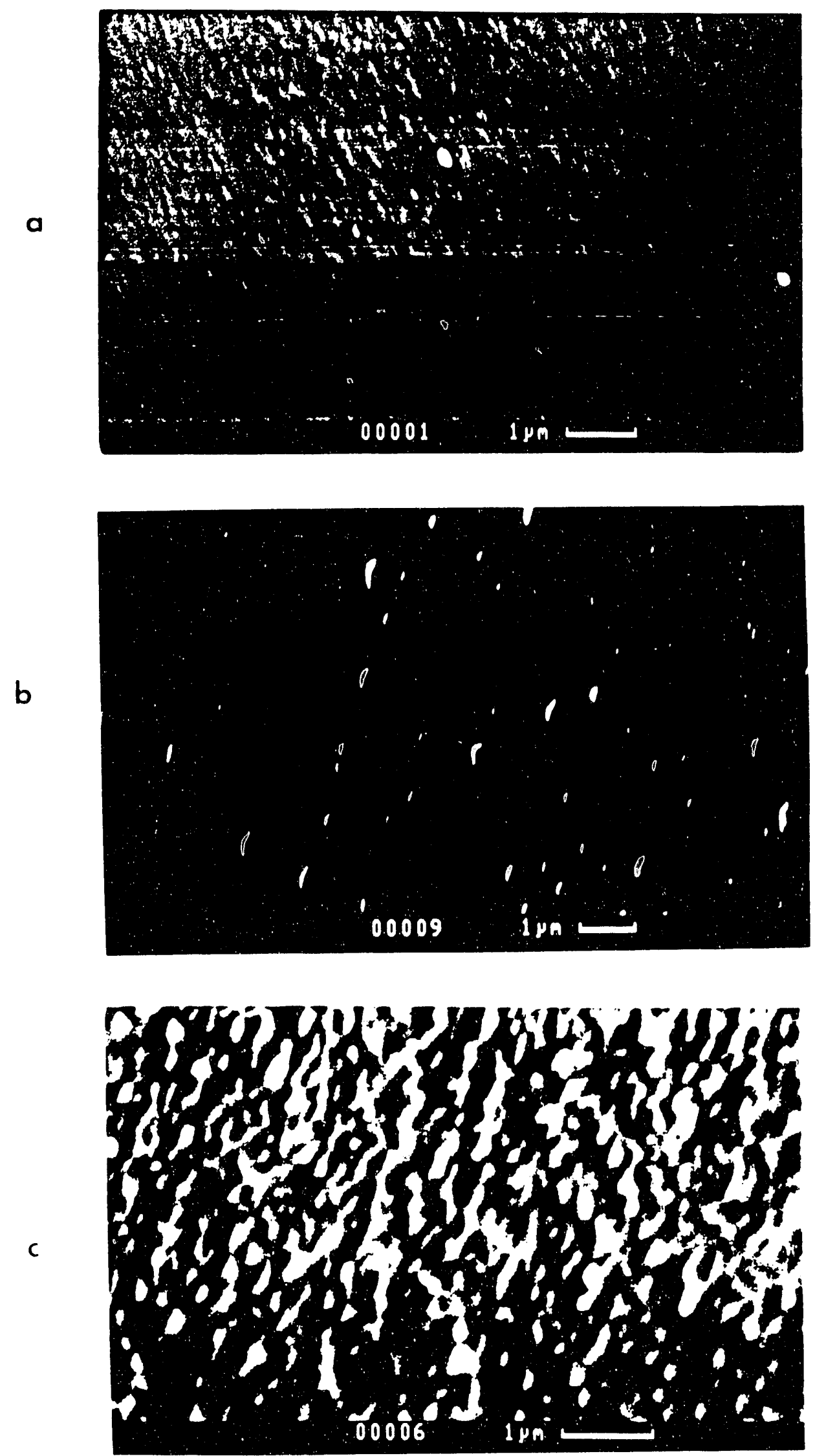

Figure 1.15. SEM micrographs of the hore surface of a Vycor lube after Si( $)_{2}$ deposition with $2.7 \% \mathrm{SiCl}_{4}$ and $1.3 \% \mathrm{H}_{2}()$ at $6(x) " \mathrm{C}$. Deposition time wais 15 minutes (a), $3($ ) minutes (h), $5($ ) minutes (c). 

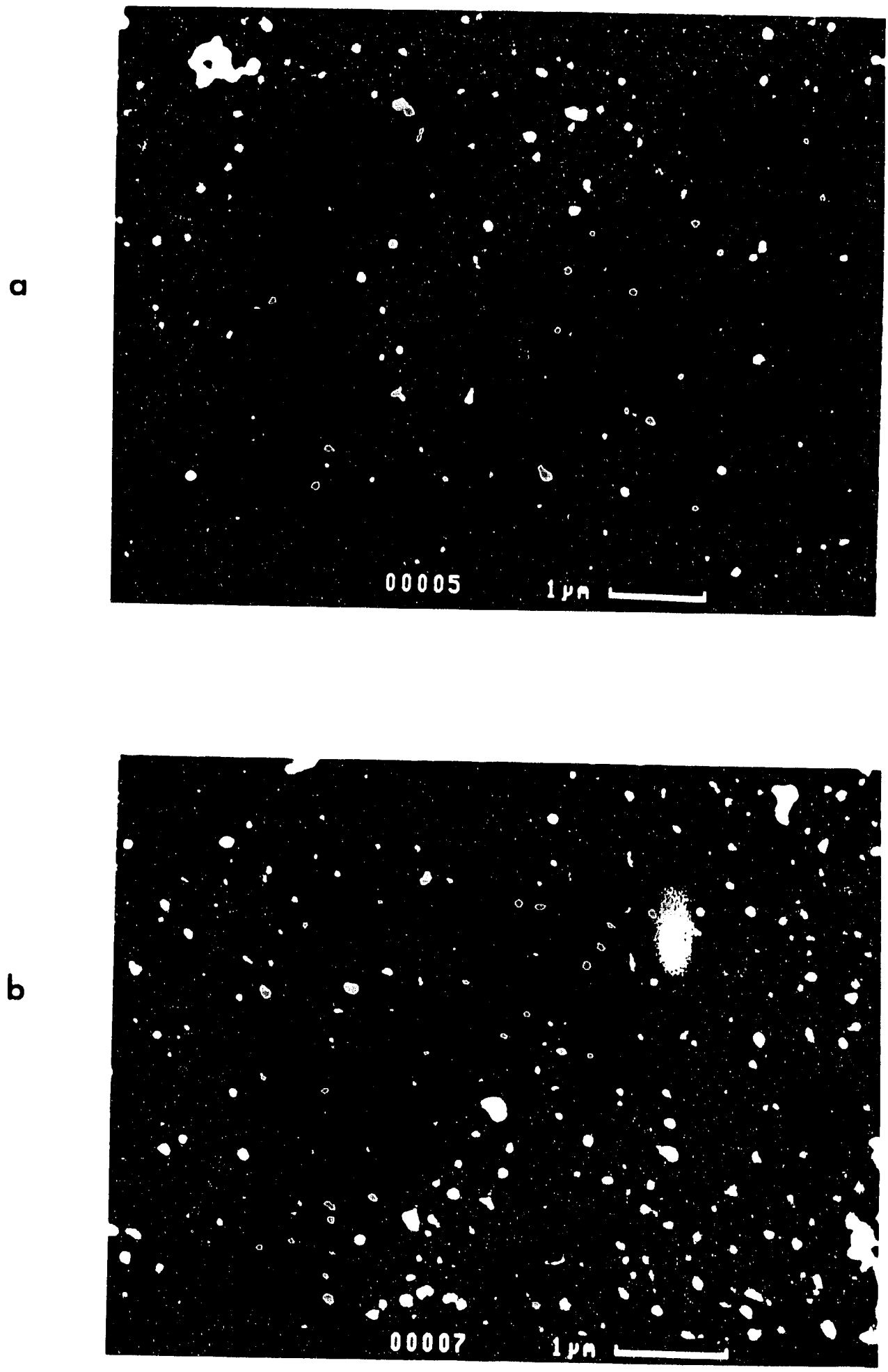

Figure 1.16. SEM micrographs of the bore surface of a Vycor tube after Sio deposition using $8.7 \% \mathrm{SiCl}_{4}$ and $1.3 \% \mathrm{H}_{2} \mathrm{O}$ at $6(0)$ () $\mathrm{C}$ for $3(1)$ minutes (immediately after pore plugging). 


\section{Chapter 2}

Membrane Stability Testing 


\subsection{Introduction}

Silica films produced by silane $\left(\mathrm{SiH}_{4}\right)$ oxidation are known to undergo densification upon prolonged annealing at high temperatures. ${ }^{1,2}$ In the presence of water vapor this densification is more severe such that heating in steam for 10 hours at $450^{\circ} \mathrm{C}$ of films deposited below $450^{\circ} \mathrm{C}$ by $\mathrm{SiH}_{4}$ oxidation decreased the film thickness by $8 \%$ from the as deposited value. ${ }^{3}$

In a previous project at Caitech ${ }^{4}$ we had studied the stability of $\mathrm{SiO}_{2}$ membranes prepared at $450^{\circ} \mathrm{C}$ by $\mathrm{SiH}_{4}$ oxidation in the opposing reactants geometry. This oxidation was carried out both under dry conditions and in the presence of water vapor. Subsequent dry annealing of the membranes reduced slowly the $\mathrm{H}_{2}$ and $\mathrm{N}_{2}$ permeances. Annealing in a $\mathrm{H}_{2} \mathrm{O}-\mathrm{N}_{2}$ atmosphere reduced more sharply the $\mathrm{H}_{2}$ permeance. But the $\mathrm{N}_{2}$ permeance increased or decreased depending on whether the deposition had been carried out in the absence of presence of water.

In this project the preparation of silica membranes was carried out at temperatures above $600^{\circ} \mathrm{C}$ and under substantial partial pressures of water vapor. However, the deposition reaction lasted only a few minutes to one hour. It was important, therefore, to examine the stability of the membranes under extended exposure to a gas with a composition similar to that anticipated in a membrane reactor for the water gas shift reaction. For this purpose a number of stability tests were carried out as described below. The first two series of these tests were conducted with a simulated coal gas (including $\mathrm{H}_{2} \mathrm{~S}$ ). The remaining two series of tests were conducted with $\mathrm{H}_{2} \mathrm{O}-\mathrm{N}_{2}$ mixtures, since other than $\mathrm{H}_{2} \mathrm{O}$ none of the components of the coal gas was expected to have a significant effect on the annealing process.

The stability testing conducted as part of this project did not examine the effect of trace contaminants in the coal gas. Among these contaminants alkali metal vapors can have a long term deleterious effect by gradually dissolving in the glass structure and reducing the permeance. ${ }^{5}$ The content of alkali vapor in the feed to the membrane 
reactor would depend on the particular coal and on the preceding particulate removal scheme. A guard bed of silica, silica-alumina, etc. particles could be used to reduce further, if necessary, the alkali content of the gas before introduction in the membrane reactor.

\subsection{Apparatus and Procedure}

The apparatus for the stability tests consists of a flanged stainless steel cylinder, $1 \mathrm{~m}$ long, $3.8 \mathrm{~cm} \mathrm{ID}$, connected to a Bourdon pressure gauge and to lines for introduction of various gases and for evacuation. Up to eight membrane tubes could be secured in this cylinder for an extended stability test. After the membrane tubes were placed and secured in the cylinder, an amount of liquid water placed in a crucible was introduced, calculated to produce the desired partial pressure of water at the test temperature. The cylinder was then sealed, evacuated and pressurized with the other gases, one at a time, while recording the pressure increase for each gas. The pressure increase for each gas was chosen to provide the desired partial pressure at the test temperature. After all the gases were introduced, the cylinder was slowly heated by an electrical furnace to the desired test temperature $\left(550\right.$ or $\left.600^{\circ} \mathrm{C}\right)$ and kept at that temperature for a period from one day to two weeks. At the completion of the heating period, the cylinder was slowly cooled to room temperature and opened. Each membrane tube was then tested for permeance to hydrogen and nitrogen at different temperatures. In some cases the membrane tubes were reintroduced into the pre'ssure cylinder and the procedure of heating, cooling and testing were repeated.

\subsection{Results}

First Series of Stability Tests

In the first series of stability tests seven tubes were placed in the pressure cylinder and exposed to a gas of composition $22 \% \mathrm{H}_{2}, 27 \% \mathrm{CO}, 18 \% \mathrm{CO}_{2}, 32 \% \mathrm{H}_{2} \mathrm{O}, 1 \% \mathrm{H}_{2} \mathrm{~S}$ at $12 \mathrm{~atm}$ total pressure and $600^{\circ} \mathrm{C}$ temperature for two days. Table 2.1 shows the 
permeation coefficients before and after the stability test. The results of the stability testing are summarized in Table 2.1 in terms of the $\mathrm{H}_{2}$ and $\mathrm{N}_{2}$ permeation coefficients before and after the treatment. With the exception of tube 4 containing $\mathrm{B}_{2} \mathrm{O}_{3}$ all tubes suffered significant loss of hydrogen permeance, in most cases by a factor of 5-20. Tube No. 5 underwent only a modest loss of hydrogen permeance. This tube had been prepared by $\mathrm{SiH}_{4}$ oxidation rather than $\mathrm{SiCl}_{4}$ hydrolysis. Most tubes underwent an increase in nitrogen permeance, such that the $\mathrm{H}_{2}: \mathrm{N}_{2}$ ratio declined, in some cases dramatically. Of all tubes tested only tubes 1 and 5 retained satisfactory hydrogen permeance coefficient as well as satisfactory $\mathrm{H}_{2}: \mathrm{N}_{2}$ permeance ratio. Tube No. 4 containing the $\mathrm{B}_{2} \mathrm{O}_{3}$ layer underwent an increase of hydrogen permeance and a larger increase of nitrogen permeance. The final ratio is close to the Knudsen value of 3.74 showing that the $\mathrm{B}_{2} \mathrm{O}_{3}$ layer lost its integrity during the thermal treatment.

\section{Second Series of Stability Tests}

Another series of tests was carried out with several $\mathrm{SiO}_{2}$ and $\mathrm{TiO}_{2}$ membranes that had been prepared previously under various conditions. The conditions of membrane preparation and the results of the stability tests are summarized in Tables 2.2-2.4 Table 2.2 lists the stability tests for $\operatorname{six} \mathrm{SiO}_{2}$ membranes, one prepared in the one-sided geometry by $\mathrm{SiCl}_{4}$ hydrolysis, four prepared in the opposing reactants geometry by $\mathrm{SiCl}_{4}$ hydrolysis, and one prepared in the opposing reactants geometry by $\mathrm{SiH}_{4}$ oxidation. All membranes were subjected to several days of heating at 500 or $600^{\circ} \mathrm{C}$, under $10-12$ atmospheres total pressure in a gas with $22 \% \mathrm{H}_{2}, 27 \% \mathrm{CO}, 32 \% \mathrm{H}_{2} \mathrm{O}, 18 \% \mathrm{CO}_{2}$ and $1 \%$ $\mathrm{H}_{2} \mathrm{~S}$ simulating the coal gas from oxygen-blown gasification of a high sulfur coal. In all cases the treatment resulted in a decrease of the $\mathrm{H}_{2}$ permeance and an increase of the $\mathrm{N}_{2}$ permeance. All membranes prepared by $\mathrm{SiCl}_{4}$ hydrolysis in the opposing reactants geometry underwent drastic decline of permeance after the first two days of treatment and failed after an additional two-day period. The membrane prepared by $\mathrm{SiCl}_{4}$ hydrolysis in the one-sided geometry and the one prepared by $\mathrm{SiH}_{4}$ oxidation in the opposing reactants 
geometry underwent significant decrease of the $\mathrm{H}_{2}$ permeance during the first two-day period but little further decrease during the second two-day period. The hydrogen permeance of $0.04-0.06$ at $600^{\circ} \mathrm{C}$ is lower by a factor 3-5 than the permeance measured immediately after deposition. The $\mathrm{H}_{2}: \mathrm{N}_{2}$ penneance ratios also declined from about 1000 to about $170-280$.

Examination of the results of Table 2.2 shows that all membranes prepared by $\mathrm{SiCl}_{4}$ hydrolysis in the opposing reactants geometry failed, while the membrane prepared by the same reaction in the one-sided geometry survived the stability test. To test the stability of membranes prepared in the one-sided geometry further, a fresh membrane was prepared and tested under the conditions summarized in Table 2.3. It should be noted that the deposition of this new membrane was carried out with a larger partial pressure of $\mathrm{H}_{2} \mathrm{O}$ compared with membrane No. 5 of Table 2.2 (5\% vs. $2.5 \%$ ). After deposition the membrane was exposed to a total of 7.5 days of hydrothermal treatment. Permeation measurements were carried out immediately after deposition, after 1.5 days of treatment, after 3 days of treatment, and after 7.5 days of treatment. The $\mathrm{H}_{2}$ permeance at $600^{\circ} \mathrm{C}$ declined by a factor of four after the first 3 days of treatment and showed no further decline in 4.5 additional days. The final value of 0.075 at $600^{\circ} \mathrm{C}$ or 0.048 at $450^{\circ} \mathrm{C}$ appears to be close to the stable value of the fully densified membrane. The $\mathrm{H}_{2}: \mathrm{N}_{2}$ permeance ratio likewise declined from about 1000 to a final stable value of 750 . The results of this long-term stability test can be considered as satisfactory although the final permeance was low.

Similar stability tests were conducted for three $\mathrm{TiO}_{2}$ membranes prepared by $\mathrm{TiCl}_{4}$ hydrolysis in the opposing reactants geometry. It will be recalled that $\mathrm{TiO}_{2}$ membranes can be prepared only in the opposing reactants geometry. The conditions of membrane preparation and the results of the stability tests are summarized in Table 2.4. All three tubes underwent significant decline of $\mathrm{H}_{2}$ permeance after the first two days of 
treatment, but little further change after three additional days of treatment. The final stable permeance at $600^{\circ} \mathrm{C}$ was $0.04-0.07 \mathrm{~cm}^{3} / \mathrm{cm}^{2}$-min-atm, only a little lower than for the $\mathrm{SiO}_{2}$ membranes prepared in the opposing reactants geometry (Table 2.3). The $\mathrm{H}_{2}: \mathrm{N}_{2}$ permeance ratios declined from about 300 to $70-120$, substantially below those listed in Table 2.4 for the $\mathrm{SiO}_{2}$ membranes.

\section{Third Series of Stability Tests}

A new series of tests was conducted on a fresh batch of $\mathrm{SiO}_{2}$ membranes prepared in one-sided and opposing reactants geometries at different temperatures and reactant concentrations in order to test the effect of deposition conditions on membrane stability. In this series several membranes were placed in the high pressure system in contact with a gas containing $30-32 \% \mathrm{H}_{2} \mathrm{O}$ in nitrogen at 10 atmospheres total pressure and $550^{\circ} \mathrm{C}$ temperature. The permeation rates of $\mathrm{H}_{2}$ and $\mathrm{N}_{2}$ were measured at 450 and $600^{\circ} \mathrm{C}$ for the membranes as deposited, after 12 hours annealing in dry nitrogen at $750^{\circ} \mathrm{C}$, and after exposure to the aforementioned hydrothermal environment for several days as detailed in Tables 2.5-2.7 below.

Table 2.5 shows the results of the stability tests for three membranes deposited in the opposing reactants geometry. After two days of hydrothermal treatment, the permeance of all three membranes declined by a factor of ten to a very low value of $0.003-0.005 \mathrm{~cm}^{3} / \mathrm{cm}^{2}$-min-atm. No further tests were conducted with these membranes.

The permeance of five membranes prepared by one-sided deposition under different conditions is listed in Table 2.6. After 9 days of hydrothermal treatment $\left(550^{\circ} \mathrm{C}\right.$ under 3 atm of water vapor), all membranes underwent significant decline of permeance, but membranes $16,17,18$, and 20 reached respectable hydrogen permeances of about $0.07-0.12 \mathrm{~cm}^{3} / \mathrm{cm}^{2}-\mathrm{min}$-atm. Two of these membranes (16 and 18 ) were later damaged in handling. The remaining two membranes 17 and 20 were subjected to 12 additional days of Irothermal treatment. As detailed in Table 2.7, membrane 17 underwent some 
decline of permeance, but membrane 20 showed no further decline of permeance after this additional treatment.

\section{Fourth Series of Stability Tests}

A fourth series of tests was conducted with silica membranes coded 24-30 prepared by one-sided deposition as defined in Table 1.2 of the preceding chapter. It will be recalled that this batch of membranes was prepared using two different types of support tubes and three different silica reagents. The permeances of these membranes immediately after deposition, after twelve hours of thermal treatment at $700-750^{\circ} \mathrm{C}$, and after thirteen days of additional hydrothermal trestment at $550^{\circ} \mathrm{C}$ under 3 atm of water vapor are listed in Table 2.8. All membranes prepared in this series underwent hydrothermal treatment with the expected decrease of hydrogen permeance, but no catastrophic change occurred as with the membranes of the first series. Using the dimer and trimer silica precursors gave only a slight improvement in hydrogen permeance and had no consistent effect on $\mathrm{H}_{2}: \mathrm{N}_{2}$ selectivity. Using the $25 \AA$ pore tubes improved moderately the hydrogen permeance but seemingly reduced the selectivity from about 500-1000 to about 200-300. The best permeance was obtained with membrane 29 made with $25 \AA$ pore size tubes using the dimer precursor. This tube had hydrogen permeance of $0.13 \mathrm{~cm}^{3} / \mathrm{cm}^{2}$-min-atm with $\mathrm{H}_{2}: \mathrm{N}_{2}$ selectivity of 200 at $500^{\circ} \mathrm{C}$, after the thirteen-day hydrothermal treatment. As stated earlier, the measurement of the very small nitrogen permeance is subject to considerable error from small leaks in the system. Hence the true $\mathrm{N}_{2}$ permeances may be considerably lower than the measurements indicate, and the calculated selectivities probably significantly underestimate the true selectivities. Activation energies for hydrogen permeance varied somewhat from membrane 10 membrane but were generally in the range $25-30 \mathrm{~kJ} / \mathrm{mol}$ with the exception of membrane 25 which gave $39 \mathrm{~kJ} / \mathrm{mol}$.

Figure 2.1 shows a sharp increase in the activation energy following deposition and hydrothermal treatment. The permeance of the untreated tuhe has negative activation 
energy in accor'ance with Knudsen diffusion (permeance inversely proportional to $T^{1 / 2}$ ). After depositi nd annealing, the activation energy increases to a value about $10 \mathrm{~kJ}$. This value is the result of the series combination of the deposit layer resistance and the resistance of the un eated tube. After the hydrothermal treatment, the resistance is dominated by the deposit layer, whence the much higher activation energy $(\sim 30 \mathrm{~kJ} / \mathrm{mol})$.

In addition to the stability tests listed in Table 2.8 , a membrane from a previous series (No. 20 of Table 2.6) which had undergone 9 days hydrothermal treatment was subjected to additional 13 days of the same treatment $\left(550^{\circ} \mathrm{C}, 3 \mathrm{~atm}\right.$ of water vapor) resulting in only $8 \%$ decrease of its hydrogen permeance and no change of selectivity.

\section{Discussion}

The results of the preceding series of hydrothermal testing suggest that membrane stability is quite sensitive to the preparation conditions. Membranes produced by $\mathrm{SiCl}_{4}$ hydrolysis in the opposing reactants geometry in most cases cracked during extended hydrothermal treatment. Membranes prepared in the one-sided geometry with $\mathrm{SiCl}_{4}$ partial pressure above $0.1 \mathrm{~atm}$ also often cracked upon cooling and reheating. The most stable membranes were those prepared with low partial pressures of $\mathrm{SiCl}_{4}$ (below 0.1 atm) at $700-750^{\circ} \mathrm{C}$ deposition temperature. These membranes withstood as long as three weeks of heating $\left(550^{\circ} \mathrm{C}\right.$ with 3 atm $\mathrm{H}_{2} \mathrm{O}$ vapor, 7 atm $\left.\mathrm{N}_{2}\right)$ including one or two intermediate cooling and reheating periods. These membranes also turned out generally to have higher hydrogen permeance than the other membranes. SEM results discussed in the previous chapter show that one-sided $\mathrm{SiO}_{2}$ deposition with high $\mathrm{SiCl}_{4}$ partial pressures causes homogeneous formation of particles which accumulate on the cylindrical surface outside of the pores of the support tube. The external $\mathrm{SiO}_{2}$ layer formed under these conditions seems to cause catastrophic thermal expansion/contraction stresses during cooling and reheating. At low $\mathrm{SiCl}_{4}$ concentrations the homogeneous generation of particles is slower and the formation of an external layer limited, with the deposit layer 
being confined mainly within the pores of the support. Such thin internal layers evidently do not cause cracking of the support tube upon cooling and reheating.

\section{Literature Cited}

1. Swaroop, B., in "Thin Film Dielectrics," Vratny (Ed.), The Electrochemical Soc., New York (1969).

2. Pliskin, W. A., J. Vac. Sci. Technol. 14, 1064 (1977).

3. Kern, W., RCA Review 37, 55 (1976).

4. Nam, S. W. and Gavalas, G. R., AJChE Symp. Ser. 85, 68 (1989).

5. Shelby, J. E., "Molecular Solubility and Diffusion" in Treatise on Materials Science and Technology, M. Tomozawa and R. H. Doremus (Eds.), Vol. 17, Academic Press, 1979. 


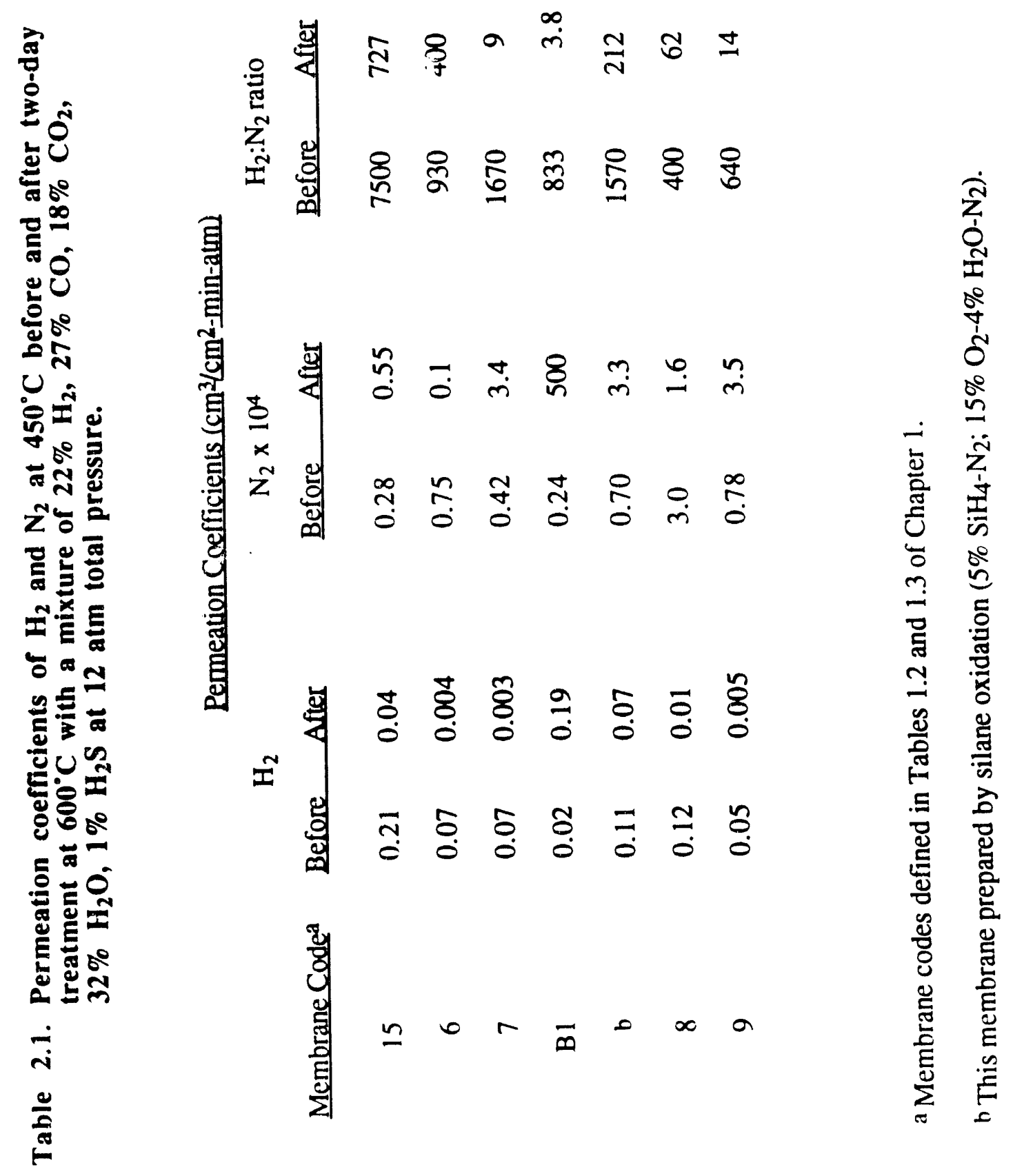




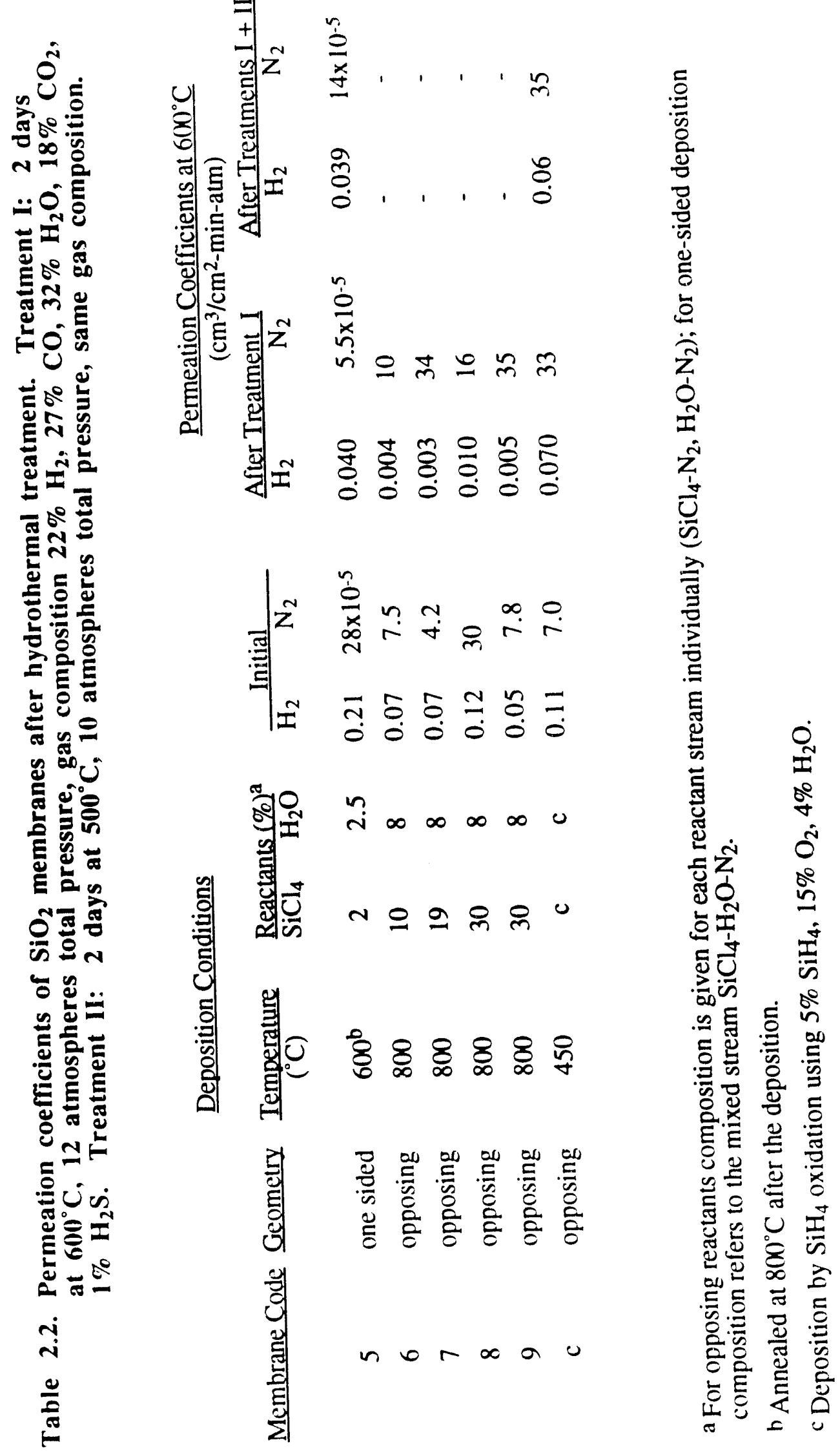


Table 2.3. Permeation coefficients of a $\mathrm{SiO}_{2}$ membrane after hydrothermal treatment. The membrane was prepared in the one-sided geometry at $600^{\circ} \mathrm{C}$ with $2 \% \mathrm{SiCl}_{4}$ and $5 \% \mathrm{H}_{2} \mathrm{O}$.

\section{Permeation Coefficients at $600^{\circ} \mathrm{C}$}

$$
\left(\mathrm{cm}^{3} / \mathrm{cm}^{2}\right. \text {-min-atm) }
$$

Treatment

None

I

I + II

I + II + III

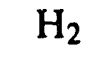

0.310

0.083

0.075

$0.075\left(0.048^{\mathrm{a}}\right)$
$\mathrm{N}_{2}$

$3 \times 10^{-4}$

2

1

$1\left(0.4^{\mathrm{a}}\right)$

I 1.5 day at $550^{\circ} \mathrm{C}, 10$ atm total pressure, gas composition $23 \% \mathrm{H}_{2}, 27 \% \mathrm{CO}$, $32 \% \mathrm{H}_{2} \mathrm{O}, 18 \% \mathrm{CO}_{2}$.

II 1.5 additional days under same conditions as $\mathrm{I}$.

III 4.5 additonal days under same conditions as I.

${ }^{\text {a}}$ Permeation coefficients at $450^{\circ} \mathrm{C}$. 


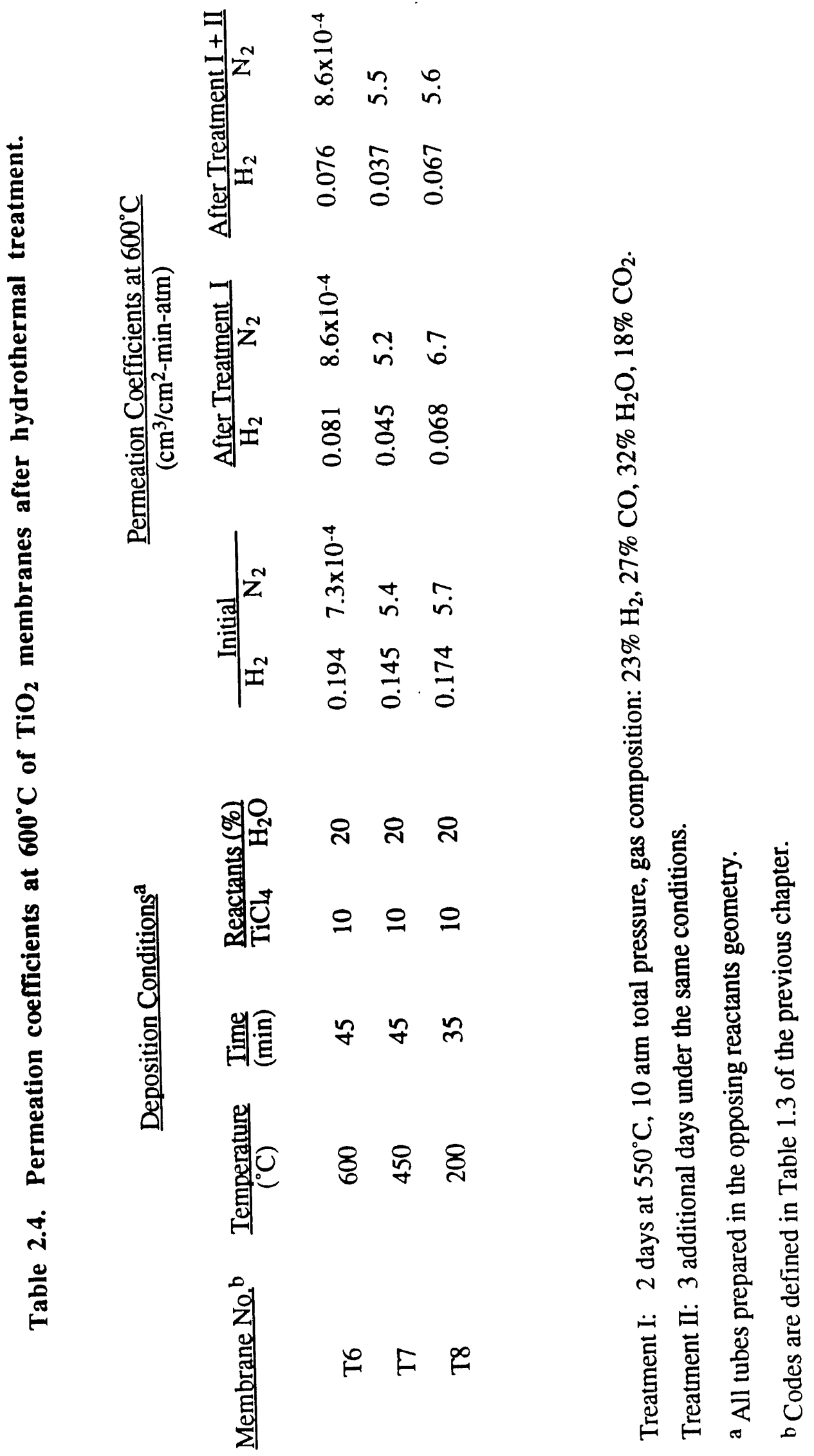




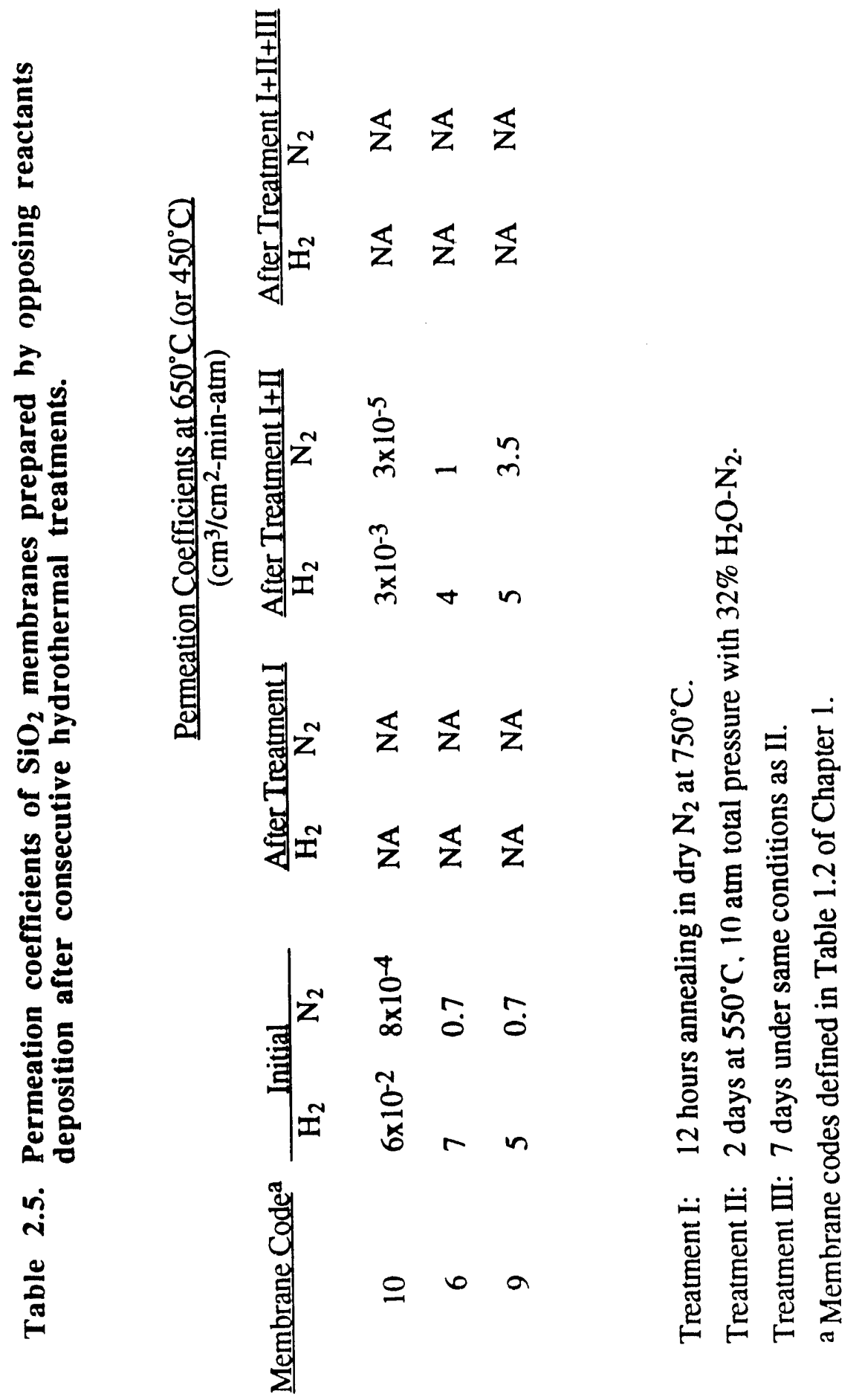




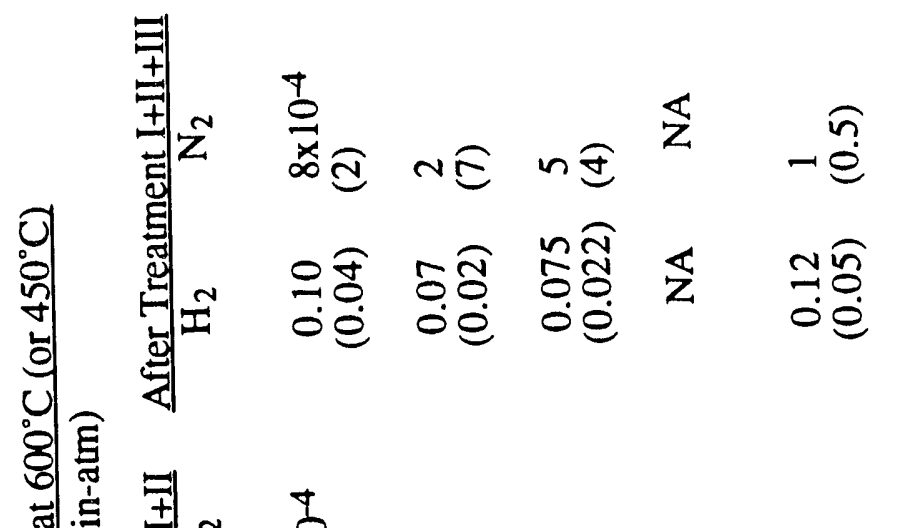

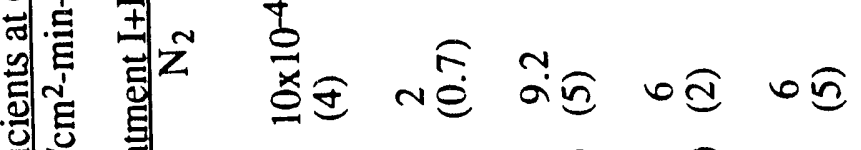

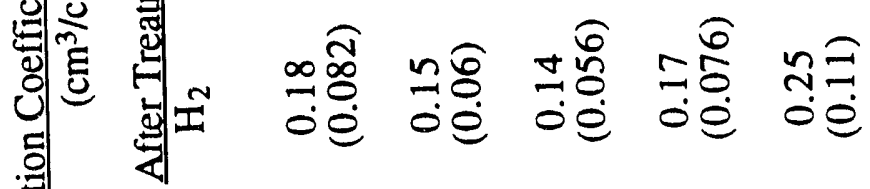

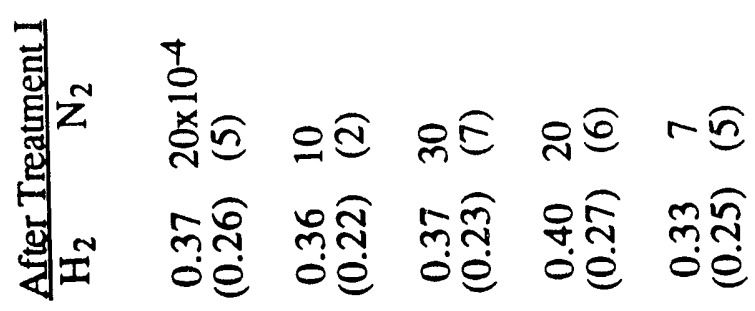

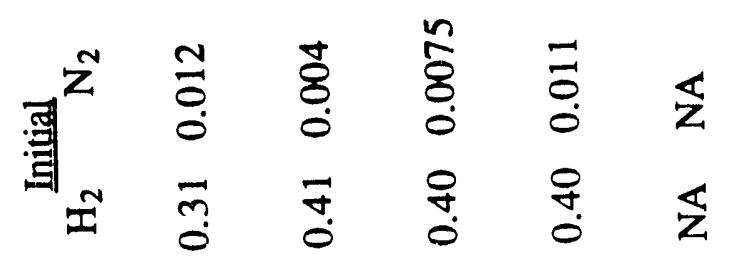
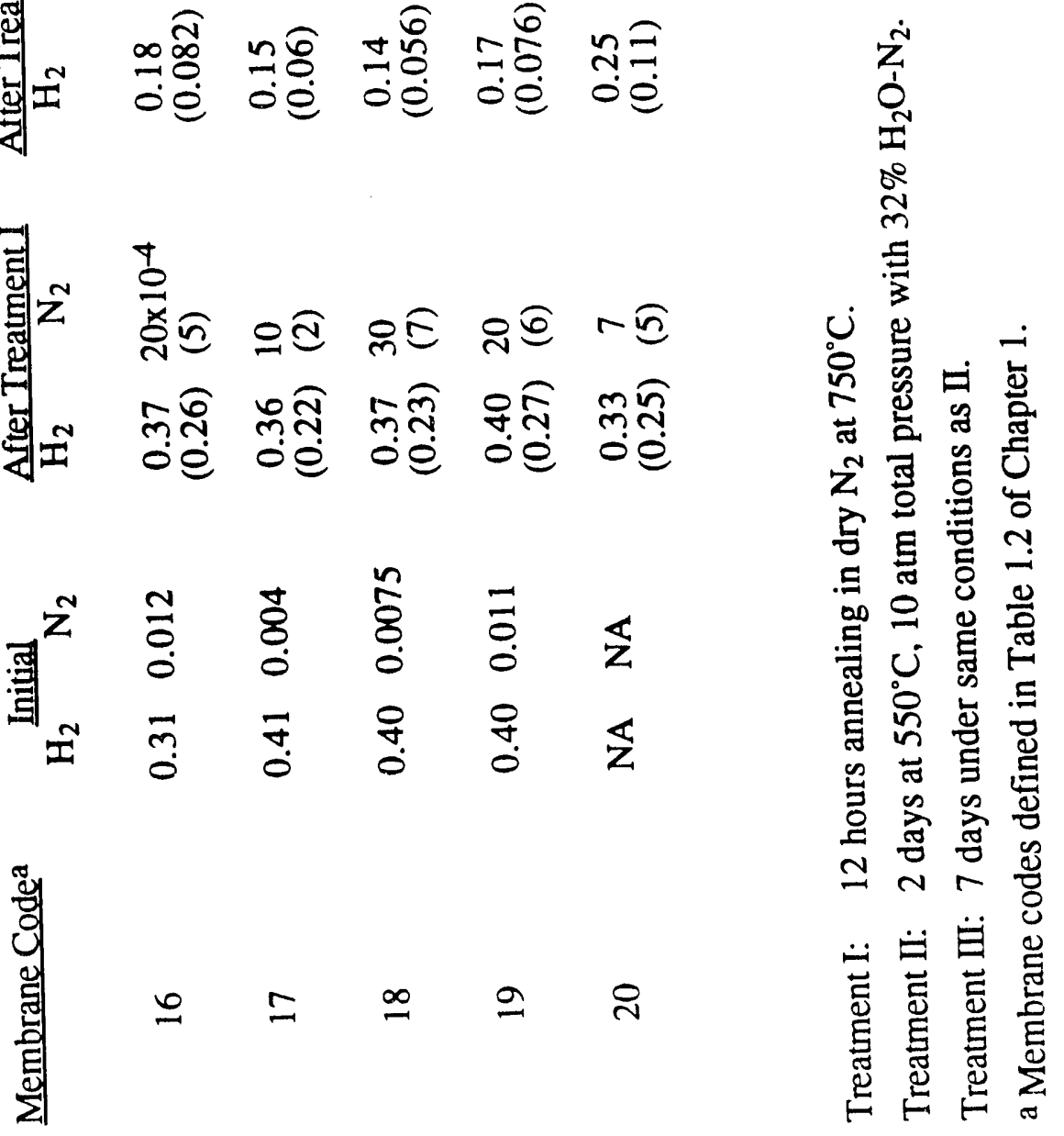


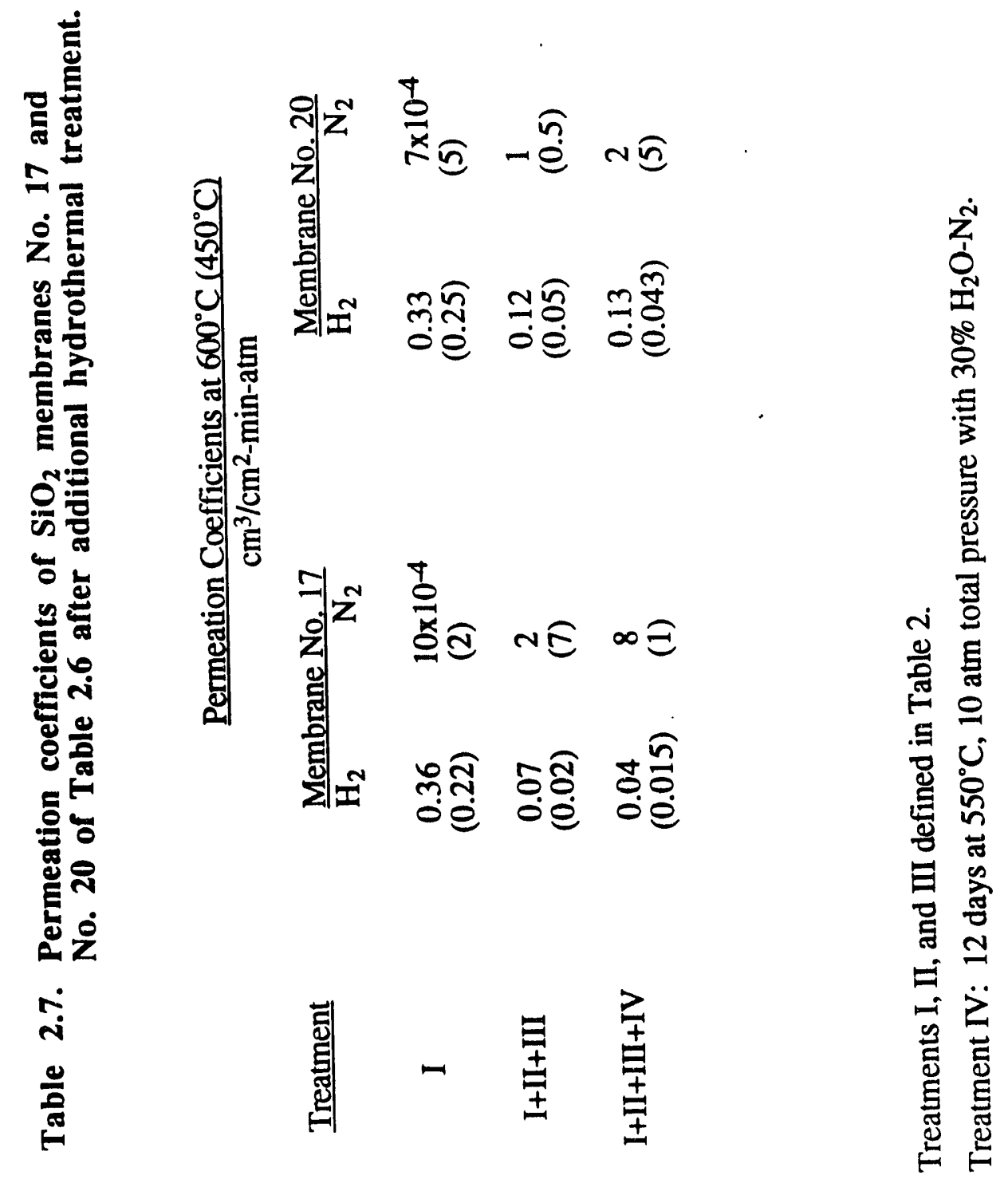




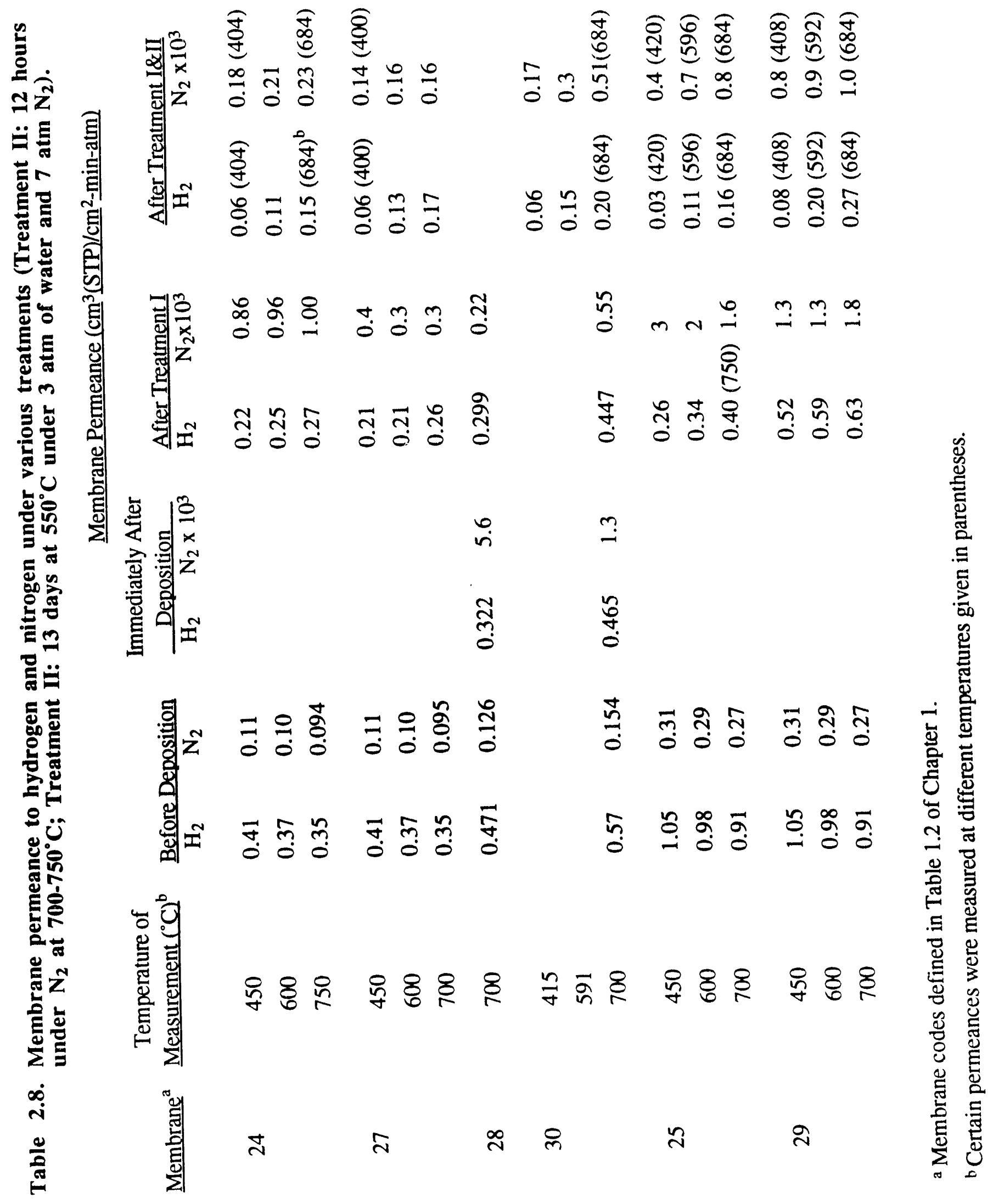




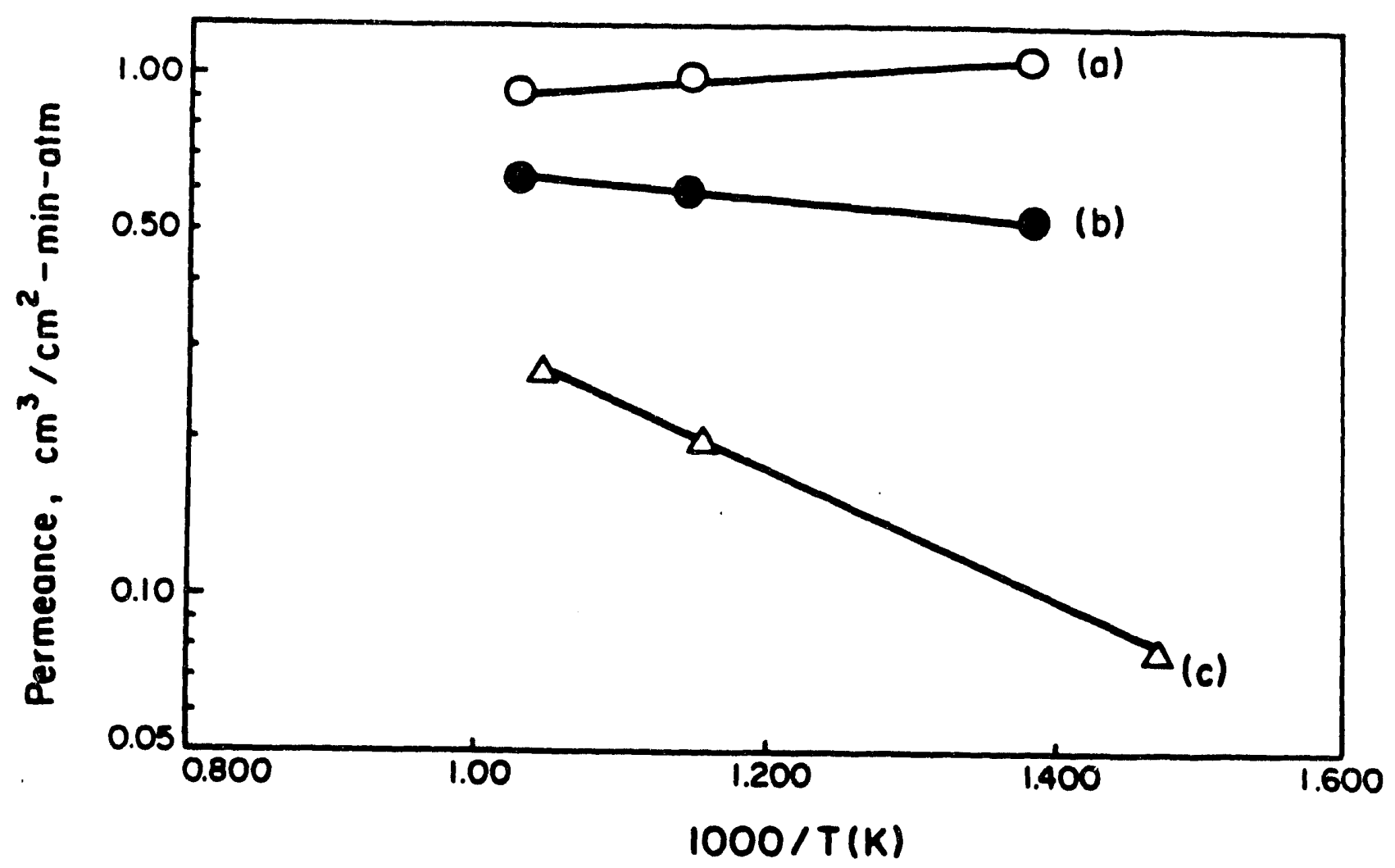

Figure 2.1. Arrhenius diagram of the hydrogen permeance of $\mathrm{SiO}_{2}$ membrane

(a) before deposition, (b) after deposition, and (c) after 13 days at $550^{\circ} \mathrm{C}$, 3 atm $\mathrm{H}_{2} \mathrm{O}$. 


\section{Chapter 3}

Analysis and Economic Evaluation of $\mathrm{SiO}_{2}$ Membrane Application to Hydrogen Production from Coal Gas 


\subsection{Introduction}

This chapter contains the report of Kinetics Technology International (KTI) (A Mannesmann Company) on work performed under subcontract to this contract. KTI made an economic evaluation of the silica membranes prepared at Caltech for the case of an ammonia-from-coal process. This process was chosen to take advantage of the capability of the silica membranes to produce high purity hydrogen as required for ammonia synthesis. The process analysis and economic evaluation conducted by KTI was based on information on membrane properties and on the operation of the conventional and membrane shift reactors generated at Caltech. An appendix to this chapter describes a membrane shift reactor model developed at Caltech for this purpose.

The KTI study was performed by Mrssrs. R. R. Jungerhans, Manager of Technology Development and Pietro Brandani, Process Engineer. Mr. William Campbell of M. W. Kellog provided information on the composition of coal gas produced by a KRW gasifier and made useful suggestions about power cogeneration associated with the process under consideration.

\subsection{Description of the Membrane Shift Reactor}

The membrane shift reactor is shown schematically in Figure 3.1. It consists of three fixed bed sulfur resistant shift reactors $R-1, R-2, R-3$, three membrane units $M-1$, $\mathrm{M}-2, \mathrm{M}-3$, and two heat exchangers, $\mathrm{H}-1$ and $\mathrm{H}-2$ for generation of medium pressure steam. The reactors, membrane units and heat exchangers are interconnected for feed, permeate and non-permeate gas streams as shown. One typical membrane module is schematically shown in Figure 3.2. A membrane module consists of a steel casing containing the capillaries. The bottom end of each capillary is plugged, while the upper end is connected to the plenum through a glass plate. The shell space of each membrane module is kept at a pressure of 20 to $30 \mathrm{~atm}$ in order to provide sufficient $\mathrm{H}_{2}$ partial pressure driving force for hydrogen permeation across the membra Hydrogen 
permeate at about 2 atm pressure llows but of the plenum of each module to common hydrogen lines. Details of the membrane module of this study are given below.

\section{Sizing and Capital Cost Estimates of Membrane Units}

For the purpose of this study, the membrane capillaries are specified to have $0.5 \mathrm{~mm} \mathrm{ID}, 0.7 \mathrm{~mm} \mathrm{OD}$, with a deposited $\mathrm{SiO}_{2}$ layer near the outside surface. The apparent Vycor glass density is $1.5 \mathrm{gr} / \mathrm{cm}^{3}$. One square meter of outside membrane area has a capillary length:

$1 /$ pi $\times 0.7 \times(10)^{-3}=454.5 \mathrm{~m} / \mathrm{m}^{2}$

and a mass: $\mathrm{pi} / 4 \times(0.7 \times 0.7-0.5 \times 0.5) \times(10)^{-2} \times 4550 \times 1.5=128.6 \mathrm{gr}$ or $0.1286 \mathrm{~kg} / \mathrm{m}^{2}$.

The cost of the capillaries of that size has been very roughly estimated by Corning to be 300 to $600 \mathrm{USD} / \mathrm{lb}$. We shall use $500 \mathrm{USD} / \mathrm{lb}$ for our evaluation, corresponding to:

$$
0.1286 \times 2.2046 \times 500=142 \mathrm{USD} / \mathrm{m}^{2}
$$

The required membrane area has been estimated by Dr. Gavalas for two scenarios as follows:

Scenario 1 is based on a hydrogen permeance of $0.1 \mathrm{~cm}^{3}(\mathrm{STP}) / \mathrm{cm}^{2}$-min-atm, which is the permeance at a baseline operating temperature of $500^{\circ} \mathrm{C}$ measured for the membranes prepared until recently in Dr. Gavalas' laboratory. This permeance is the stabilized value after hydrothermal treatment.

Scenario 2 is based upon a permeance of $0.3 \mathrm{~cm}^{3}(\mathrm{STP}) / \mathrm{cm}^{2}$-min-atm at $500^{\circ} \mathrm{C}$. There are good reasons to expect that the permeance can be increased from 0.1 to 0.3 by certain modifications of the membrane deposition process. Such modifications are currently under investigation at Dr. Gavalas' laboratory. 


\section{Membrane Area Requirements aind Cost Estimate Per Scenario 1}

The total permeate hydrogen is $4635 \mathrm{lbmol} / \mathrm{hr}$ or $35000 \mathrm{gmol} / \mathrm{min}$ and the estimated split of this flow between the three membrane units is as indicated below:

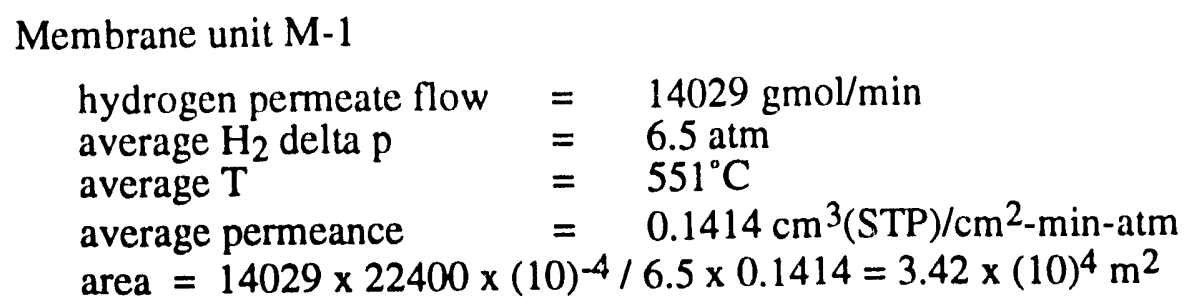

Membrane unit M-2

$\begin{array}{lll}\text { hydrogen permeate flow } & =11188 \mathrm{gmol} / \mathrm{min} \\ \text { average } \mathrm{H}_{2} \text { delta } \mathrm{p} & =4.65 \mathrm{~atm} \\ \text { average } \mathrm{T} & =543^{\circ} \mathrm{C} \\ \text { average permeance } & =0.1266 \mathrm{~cm}^{3}(\mathrm{STP}) / \mathrm{cm}^{2} \text {-min-atm } \\ \text { area }=1188 \times 22400 \times(10)^{-4} / 4.65 \times 0.1266=4.26 \times(10)^{4} \mathrm{~m} 2\end{array}$

Membrane unit M-3

$\begin{array}{ll}\text { hydrogen permeate flow } & =9855 \mathrm{gmol} / \mathrm{min} \\ \text { average } \mathrm{H}_{2} \text { delta } \mathrm{p} & =2.76 \mathrm{~atm} \\ \text { average T } & =513^{\circ} \mathrm{C} \\ \text { average permeance } & =0.1097 \mathrm{~cm}^{3}(\mathrm{STP}) / \mathrm{cm}^{2} \text {-min-atm } \\ \text { area }=9855 \times 22400 \times(10)^{-4} / 2.76 \times 0.1097=7.29 \times(10)^{4} \mathrm{~m}^{2} \\ \\ \text { Total membrane area }=(3.24+4.26+7.29) \times(10)^{4}=15 \times(10)^{4} \mathrm{~m}^{2} \\ \text { Capillaries at } 142 \mathrm{USD} / \mathrm{m}^{2}:\end{array}$

\section{Estimated Cost of Membrane Modules}

One membrane module could be made as a cylinder with $12 \mathrm{~cm}$ diameter, $30 \mathrm{~cm}$ total length containing 14700 capillaries of $25 \mathrm{~cm}$ length, occupying $50 \%$ of the module cross section, for a total of $3673 \mathrm{~m}$ capillary length or $8.08 \mathrm{~m}^{2}$ membrane area.

The cost of each module at mass production is estimated at about 100 USD. The total number of modules required is:

$$
15 \times(10)^{4} / 8.08=18600 \text { at a total cost of about } 1.9 \mathrm{MM} \text { USD }
$$

The cost of piping, valves and housing is estimated an additional 0.5 MM USD. 
The Total Cost of the Membrane Units is: $21.2+1.9+0.5=23.6$ MM USD

The membrane modules are divided into the three membrane units $M-1, M-2$ and M-3 with approximate individual volumes of 25,31 and $54 \mathrm{~m}^{3}$, each containing respectively about 4200,5200 and 9100 modules. The modules are combined in series and parallel. For example, 50 or 100 modules are connected in series in a sub unit and the required number of sub units are connected in parallel to form the unit.

\section{Cost Estimate per Scenario II}

Using the higher permeance of $0.3 \mathrm{~cm}^{3}(\mathrm{STP}) / \mathrm{cm}^{2}$-min-atm at $500^{\circ} \mathrm{C}$, instead of 0.1 as used in Scenario I and maintaining all other assumptions of Scenario I above, we obtain a total cost for the three membrane units of $23.6 / 3=7.9$ MM USD.

\subsection{Basis of the Study}

In this study two hydrogen production processes are compared as follows:

1. A conventional process with a high and low temperature sulfur resistant shift reactor with intercooling, followed by PSA purification.

2. A process utilizing a membrane shift reactor, as described above, followed by methanation.

The feedgas is produced in a KRW oxygen blown gasifier for $1000 \mathrm{~m} / \mathrm{d}$ low sulfur $(0.4 \%)$ Western (Utah) coal. It passes via ash and slag removal and enters the hydrogen production plants free of particulates.

In both processes, hydrogen is produced in an amount equivalent to $560 \mathrm{mLd}$ ammonia. The balance of the gas is used for generation of power in a gas turbine cogeneration cycle. This power is used for driving the combustion air compressor and the ammonia syngas compressor. All excess steam is converted into power and the power in excess of the needs of the schemes under consideration is exported. 
Both processes do not yield purified $\mathrm{CO}_{2}$ as required for production of urea.

The sulfur content in the gasification gas is about $0.13 \%$. In the non-permeate gas from the membrane shift reactor, it is about $0.19 \%$. It is assumed that these gases can be burned in the gas turbine without further clean-up.

\subsection{Process Description}

Coal gasification delivers the following feedgas as reported by Kellogg: (see reference page 75 ).

$\begin{array}{lcl}\mathrm{CO} & 38.63 & \text { vol\% } \\ \mathrm{H}_{2} & 28.87 & \\ \mathrm{CO}_{2} & 10.69 & \\ \mathrm{CH}_{4} & 4.99 & \\ \mathrm{~N}_{2} & 0.70 & \\ \mathrm{Ar} & 0.70 & \\ \mathrm{O}_{2} & 0.00 & \\ \mathrm{NH}_{3} & 0.38 & \\ \mathrm{H}_{2} \mathrm{~S} & 0.11 & \\ \mathrm{COS} & 0.02 & \\ \mathrm{H}_{2} \mathrm{O} & 14.99 & \\ \mathrm{HCN} & 95 \mathrm{ppm} \\ \mathrm{MeOH} & 0.00 & \\ \text { SUM } & \mathbf{1 0 0 . 0 0}\end{array}$

Total flow: $10041 \mathrm{lbmol} / \mathrm{hr}$

$\begin{array}{ll}\text { Pressure: } \quad 412 \mathrm{psig} \\ \text { Temperature: } & 1925^{\circ} \mathrm{F}\end{array}$

Membrane Shift Process

$\mathrm{CO}$ contained in the coal gasification gas is shifted towards $\mathrm{CO}_{2}$ while producing more hydrogen, and high purity hydrogen is separated in the membrane shift reactor. The permeate gas is the hydrogen rich gas stream. It contains about $1000 \mathrm{ppm} \mathrm{CO}+\mathrm{CO}_{2}$, $130 \mathrm{ppm}$ ineris $\left(\mathrm{CH}_{4}+\mathrm{N}_{2}+\mathrm{Ar}\right)$ and maximum $3 \mathrm{ppm}$ sulfur. This sulfur level is acceptable for the next processing steps of methanation and ammonia synthesis. The permeate gas pressure at 15 psig is relatively low. Therefore, more syngas compression is needed than in the conventional process. 
The non-permeate gas at a pressure of $376 \mathrm{psig}$ is used as fuel in a gas turbine for power generation. Because of its high $\mathrm{CO}_{2}$ content, the heating value of this gas is relatively low $(\mathrm{LHV}=163 \mathrm{BTU} / \mathrm{SCF})$, more over, it contains all the sulfur of the coal gasification gas. For the purpose of this study, the assumption is made that no further clean-up of this gas is necessary. The efficiency of the gas turbine after air compression is assumed to be about $25.4 \%$.

The gas composition change and subsequent products split in the membrane shift reactor, as determined by Caltech is given as follows:

Table 3.1 Composition of feed, permeate, and non-permeate (retentate) streams in membrane shift reactor.

\begin{tabular}{lccc} 
& Feed & Non-Permeate & Permeate \\
\hline $\mathrm{CO}$ vol\% & 33.59 & 19.46 & $454 \mathrm{ppm}$ \\
$\mathrm{H}_{2} \mathrm{O}$ & 26.08 & 6.91 & $283 \mathrm{ppm}$ \\
$\mathrm{H}_{2}$ & 25.10 & 11.48 & 99.86 \\
$\mathrm{CO}_{2}$ & 9.30 & 52.22 & $527 \mathrm{ppm}$ \\
$\mathrm{CH}_{4}$ & 4.34 & 7.26 & $99 \mathrm{ppm}$ \\
$\mathrm{H}_{2} \mathrm{~S}+\mathrm{COS}$ & 0.11 & 0.19 & $3 \mathrm{ppm}$ \\
$\mathrm{N}_{2}+\mathrm{Ar}$ & 1.22 & 2.04 & $28 \mathrm{ppm}$ \\
$\mathrm{NH}_{3}+\mathrm{HCN}$ & 0.27 & 0.45 & $6 \mathrm{ppm}$ \\
\cline { 2 - 3 } & 100.00 & 100.00 & 100.00 \\
\hline
\end{tabular}

The exothermal reaction heat to be removed at a temperature level high enough for medium pressure steam generation is $0.811 \mathrm{MJ} / 100 \mathrm{gmol}$ raw gas or $35 \mathrm{MMBTU} / \mathrm{hr}$.

Heat is recovered from the hydrogen containing gas and subsequently the gas is compressed in four stages to about 400 psig. Remaining $\mathrm{CO}$ and $\mathrm{CO}_{2}$ are miethanated, the gas is dried and high purity nitrogen from the air separation plant is added to obtain an ammonia syngas with $\mathrm{H}_{2} / \mathrm{N}_{2}$ ratio of $3: 1$ and a total inert content of $0.04 \%$. The 
ammonia synthesis loop operates at a pressure of about 1500 psig and the ammonia production is approximately $570 \mathrm{MT} / \mathrm{d}$.

In order to prevent inert build-up in the synthesis loop, a small stream is continuously purged from the loop and is added to the fuel for the gas turbine.

All non-permeate gas is burned in the gas turbine for the production of power. The small purge gas flow from the synthesis loop also goes to fuel.

Heat for the generation and superheat of 1250 and 470 psig high and medium pressure steam is recovered from the hot coal gasification gas, the membrane shift reactor, the main process stream and the gas turbine exhaust. The stack temperature is about $400^{\circ} \mathrm{F}$. All high pressure steam is run through a back pressure turbine for additional power generation. About $15 \%$ of the MP steam at the HP Turbine exhaust is injected into the main process stream to enhance the shift reaction. The remainder of the MP steam is run through a condensing turbine at 5 inch $\mathrm{Hg}$ for more power generation. Low temperature heat is recovered for BFW preheat.

A schematic block flow diagram is included at the end of this chapter.

\section{Conventional Process}

Starting with the same coal gasification gas as the membrane shift process, abou: $70 \%$ of it is sent to high and low temperature shift conversion. The hydrogen containing gas from the LTS is purified in a PSA, which produces 99.99 plus high purity hydrogen at a recovery of about $92 \%$. Conventional purification with acid gas removal and methanation would result in an ammonia synthesis gas with about $6 \%$ methane. With such high inerts content in the synthesis gas, the hydrogen loss via the loop purge would be 2 to 3 times as high as the PSA purge losses, which makes conventional purification impractical. The drawback of PSA purification is the recompression of its purge gas for usage as gas turbine fuel.

Hydrogen product for both processes is about equal and equivalent to an ammonia production of $560 \mathrm{MT} / \mathrm{d}$. The coal gasification gas contains about $0.15 \% \mathrm{H}_{2} \mathrm{~S}$. The high 
and low temperature shift catalysts are, therefore, sulfur resistant as for example BASF's $\mathrm{K}$ 8-11. $\mathrm{H}_{2} \mathrm{~S}$ and $\mathrm{CO}_{2}$ are removed in a PSA. High purity nitrogen from an air separation plant is added to obtain an ammonia synthesis gas with $\mathrm{H}_{2} / \mathrm{N}_{2}$ ratio of $3: 1$. The pressure at the syngas compressor inlet is about $370 \mathrm{psig}$, which is considerably higher than the permeate gas pressure of the membrane shift process. The syngas compression is accordingly lower.

The purge gas from the synthesis loop is small and theoretically approaches zero.

The remaining $30 \%$ of the coal gasification gas is used as fuel in a gas turbine for power generation. The sulfur content of the fuel gas is the same as in the coal gasification gas $(0.13 \%)$. The heating value is about $160 \mathrm{BTU} / \mathrm{SCF}$, which is comparable to the membrane shift process. Again, this gas is used in the gas turbine, without further treatment. The turbine efficiency, after combustion air compression is again assumed about $25.4 \%$. The purge gas from the synthesis loop is added to fuel in the gas turbine.

The steam generation and heat recovery is similar as in the membrane shift process as follows: 1250 and 470 psig high and medium pressure steam are generated in the hot coal gasification gas, the process gas leaving the shift reactors and using the gas turbine exhaust. The stack temperature is about $400^{\circ} \mathrm{F}$. All high pressure steam is run through a back pressure turbine for additional power generation. The total high pressure steam generation is about $80 \mathrm{MT} / \mathrm{hr}$, which is about the same as for the membrane shift process. In order to obtain a $99+\%$ CO conversion in the shift reactors, about $75 \%$ of the medium pressure steam of the high pressure turbine exhaust is injected into the main process stream. This is about five times the quantity needed in the membrane shift process. The remainder of the medium pressure steam is run through a condensing turbine at $5 \mathrm{Hg}$ for more power generation. Low temperature heat is recovered for BFW preheat.

A schematic block flow diagram is included at the end of this chapter. 


\subsection{Comparison of Processes}

Both processes have been evaluated on the same basis with the same efficiencies for the gas turbine, the steam turbines, similar stack temperature, etc. The ammonia synloop for both processes is practically identical, and in this report, is not considered as a contributing factor to the capital and/or operating cost difference between the two processes.

In terms of overall efficiency, the processes compare as follows:

Table 3.2 Comparison of operating characteristics of the membraneassisted and conventional process.

\begin{tabular}{llrc} 
& & Membrane & Conventional \\
\hline Feedgas & lbmol/hr & 10041 & 10041 \\
$\mathrm{NH}_{3}$ & $\mathrm{MU} / \mathrm{d}$ & 560 & 555 \\
Export power & $\mathrm{MW}$ & 44.4 & 36.6 \\
or in & $\mathrm{MM} \mathrm{BTU/hr}(\mathrm{LHV})$ & & \\
& & & \\
Feedgas & $@ 250 \mathrm{Btu} / \mathrm{SCF}$ & 953 & 953 \\
$\mathrm{NH}_{3}$ & $@ 8000 \mathrm{Btu} / \mathrm{lb}$ & 419 & 415 \\
Export power & $@ 3412 \mathrm{Btu} / \mathrm{kWhr}$ & 151 & 125 \\
Efficiency \% & & 60 & 57 \\
\hline
\end{tabular}

Starting with the same gasification gas the battery limits products of both processes are as follows:

Membrane Conventional

Ammonia product

MT/d

560

555

Export power

MW
44.4

36.6 
The power production for both processes is itemized as follows:

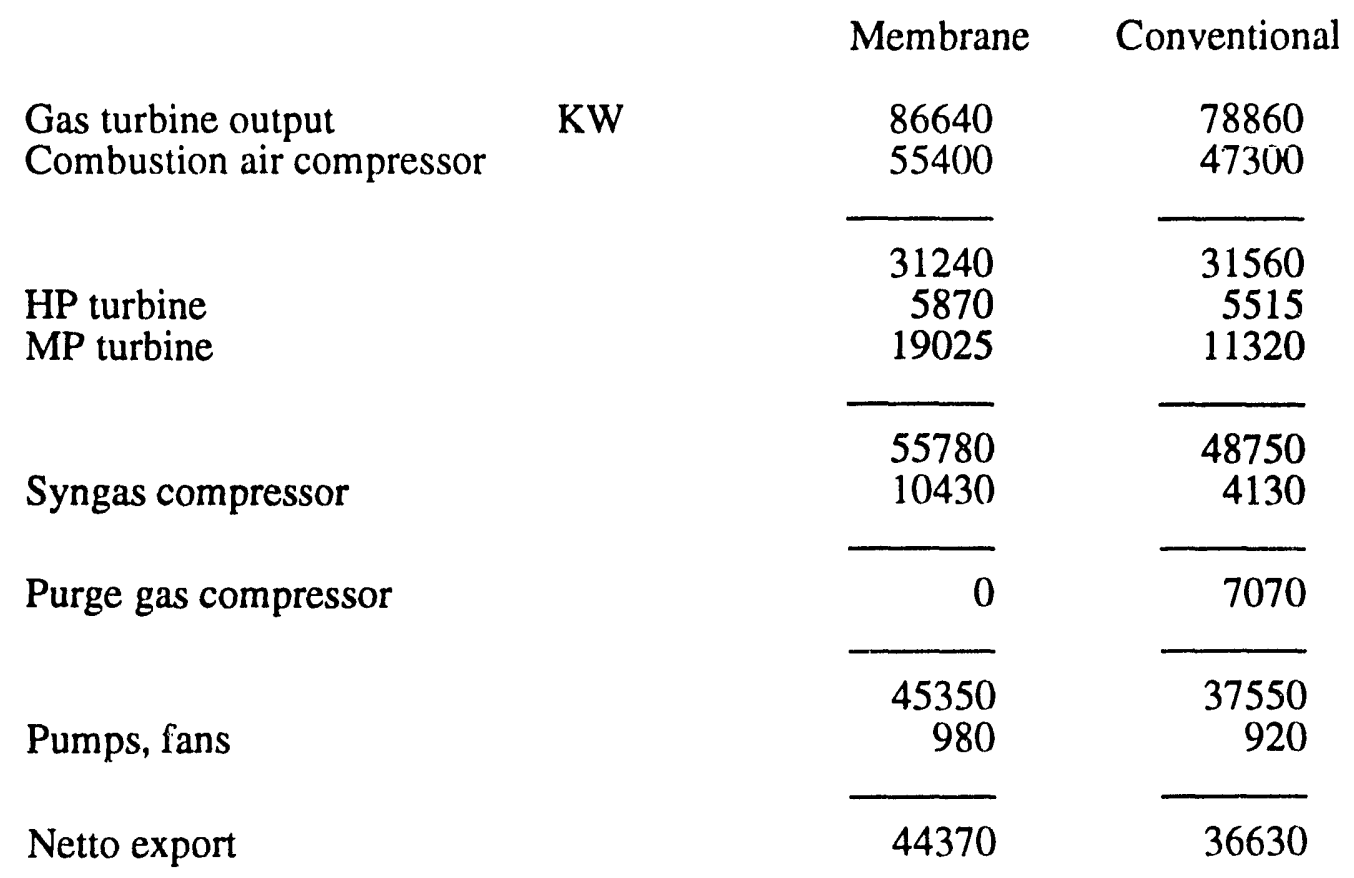

Note the following differences between the operations:

The net output of the gas turbine, after combustion air compression and the HP steam turbine outputs are about the same. The membrane process takes much less MP pressure steam and therefore, the MP steam turbine output for the membrane process is larger than for the conventional process.

As the pressure of the purified hydrogen for the membrane process is lower than in the conventional process, the syngas compressor power for the membrane process is larger than for the conventional process. These two effects are counteracting and the resulting power balance at this point is about equal.

In the conventional process, the PSA purge gas needs to be recompressed to the 400 psig level, whereas, in the membrane process, the pressure drop of the non-permeate gas in the membrane reactor is small and no recompression is necessary. As smaller power users for both processes are comparable, the nett power export for the membrane 
process is about $7.7 \mathrm{MW}$ more than for the conventional process, which is roughly equal to the power of the purge gas compressor of the conventional process.

Depending on the $\mathrm{kWhr}$ unit price, the operating cost advantage of the membrane process over the conventional process is:

$\begin{array}{lll}\text { for } & 2 \mathrm{ct} / \mathrm{kWhr}: & 1.3 \mathrm{MM} U S D / \mathrm{yr} \\ \text { for } & 5 \mathrm{ct} / \mathrm{kWhr}: & 3.1 \mathrm{MM} \text { USD/yr }\end{array}$

\section{Capital Costs of Membrane Shift Reactors}

Professor Gavalas has developed two scenarios for the cost of the membrane units as follows:

$\begin{array}{lrr}\text { Scenario I } & \text { MM USD } & 23.6 \\ \text { Scenario II } & & 7.9 \\ & & \\ \text { Difference: } & & \mathbf{1 5 . 7}\end{array}$

Scenario I is based upon currently available permeances. Senario II is for permeances expected to result from improvements in the deposition process.

With an operating advantage for the membrane between 1 and $3 \mathrm{MM} \mathrm{USD/yr,}$ depending on the $\mathrm{kWhr}$ unit price, the best simple pay out for the extra cost of Scenario I is between 5 and 15 years, which is too long a period. Scenario I appears to be too costly.

We consider Scenario II for the Membrane Shift process of this study and have the following comparisons with the conventional process:

Equipment of one process, which does not exist in the other:

- Membrane Process:

- Membrane shift reactor, including membrane units/modules shift reactors, and heat exchangers

- Methanator

- Dryer

- MP turbine (larger capacity)

- Conventional Process:

- HTS

- LTS

- Exchangers HX-1, HX-11

- PSA 
The cost of the larger syngas compressor of the membrane process is about equal to the cost of the smaller syngas compressor of the conventional process plus its purge gas compressor.

The overriding importance for the economics of the membrane process is the cost of the Vycor glass tubes which is quoted to be between 300 and $600 \mathrm{USD} / \mathrm{lb}$. As basis for this study, $500 \mathrm{USD} / \mathrm{lb}$ is taken. This is very expensive material. To view this number in perspective, the following unit prices are given for comparison:

\section{Gold 350 USD/ounce or 5000 USD/lb \\ Silver $\quad 4$ USD/ounce or $58 \mathrm{USD} / \mathrm{lb}$}

In other words, the membrane glass unit price turns out to be the geometric average of silver and gold!

The estimated installed capital difference for the two processes, being Membrane Scenario II vs Conventional, based on the above indicated equipment, is as follows:

$\begin{array}{lrr}\text { Membrane } & \text { MM USD } & 11 \\ \text { Conventional } & 6 \\ \text { Difference } & & 5\end{array}$

For the membrane process, about $80 \%$ of its cost is contributed to the membrane units; for the conventional process the PSA is the main contributor.

As stated before, the cost of the membrane units depends heavily on the Vycor glass unit price. The PSA has a capacity of about $40 \mathrm{MM} \mathrm{SCF} / \mathrm{d}$ of $\mathrm{H}_{2}$. Its estimated price is fairly accurate.

The estimated capital extra for the membrane process of about 5 MM USD is paid back by the extra power generated in this process, in approximately 1.5 to 4 years, depending on the assumed power unit price. 


\subsection{Process Optimization}

There are opportunities to improve the membrane process by optimizing its operating conditions. For instance, so far the membrane process has been evaluated with a 2 atm abs. permeate pressure. If this pressure is lowered to $1 \mathrm{~atm}$ abs. the driving force of the hydrogen through the membrane goes up and the membrane surface and its cost are reduced accordingly. However, the cost of the syngas compression increases.

This particular optimization of the permeate pressure works out as follows for Scenario II:

Membrane unit M-1

$$
\begin{aligned}
& \text { average } \mathrm{H}_{2} \text { delta } \mathrm{p}=7.5 \mathrm{~atm} \\
& \text { average permeance }=0.1414 \times 3=0.4242 \\
& \text { area }=14029 \times 22400 \times(10)^{-4} / 7.5 \times 0.4242=0.99 \times(10)^{4} \mathrm{~m}^{2}
\end{aligned}
$$

Membrane unit M-2

average $\mathrm{H}_{2}$ delta $\mathrm{p}=5.65 \mathrm{~atm}$ average permeance $=0.1266 \times 3=0.3798$

area $=11188 \times 22400 \times(10)^{-4} / 5.65 \times 0.3798=1.17 \times(10)^{4} \mathrm{~m}^{2}$

Membrane unit M-3

average $\mathrm{H}_{2}$ delta $\mathrm{p}=3.76 \mathrm{~atm}$

average permeance $=0.1097 \times 3=0.3291$

area $=9855 \times 22400 \times(10)^{-4} / 3.76 \times 0.3291=1.78 \times(10)^{4} \mathrm{~m}^{2}$

Total membrane area $=(0.99+1.17+1.78) \times(10)^{4}=3.9 \times(10)^{4} \mathrm{~m}^{2}$

\section{Capillaries cost at $142 \mathrm{USD} / \mathrm{m}^{2}: 5.6 \mathrm{MM}$ USD}

Total cost of membrane units including piping module cost, etc.:

$$
5.6+0.5+0.1=6.2 \mathrm{MM} \mathrm{USD}
$$

Cost savings over membrane operating at 2 atm abs.: $7.9-6.2=1.7$ MM USD

The increase of the operating costs of the syngas compressor is one extra stage of about $1600 \mathrm{~kW}$. With this modification, the extra capital of the membrane process is 3.3 MUSD, and the extra net power produced is $6 \mathrm{MW}$. Dependins on the price of power, the extra capital investment for the membrane will pay for itself in $1.3-3.4$ years. 


\subsection{Summary and Conclusions}

In this study we have compared two processes for production of hydrogen.

1. A novel process with a membrane/shift reactor

2. A conventional process with high and low temperature shift conversion plus PSA purification.

Hydrogen product is converted into ammonia at a capacity of $560 \mathrm{MT} / \mathrm{d}$. Unrecovered hydrogen is used for power generation.

The membrane shift reactor employs special glass membrane tubes, whose performance is still being tested at Caltech. For an optimistic performance scenario of the membrane tubes, we have estimated that the extra investment of the membrane process is about 3.3 MM USD more than the investment of the conventional process at the same ammonia production capacity. Excess power of the membrane process is about $6 \mathrm{MW}$ more than of the conventional process. Depending on the assumed power unit price as basis for the evaluation, the extra investment for the membrane process pays for itself in 1.3 to 3.4 years.

The economics of the membrane process will greatly inprove when the costs of the Vycor glass tubes come down.

Optimization of the operating conditions of the membrane process will provide opportunities to improve the economics of this novel process.

\subsection{Reference}

Private communication of W. M. Campbell of M. W. Kellogg Company to G. R. Gavalas: Material balance of the gasifier section of a coal to ammonia plant using KRW oxygen blown gasifier (Coal based KRW case Rev 0, August 3, 1990, stream 7: gasifier product to quench). 


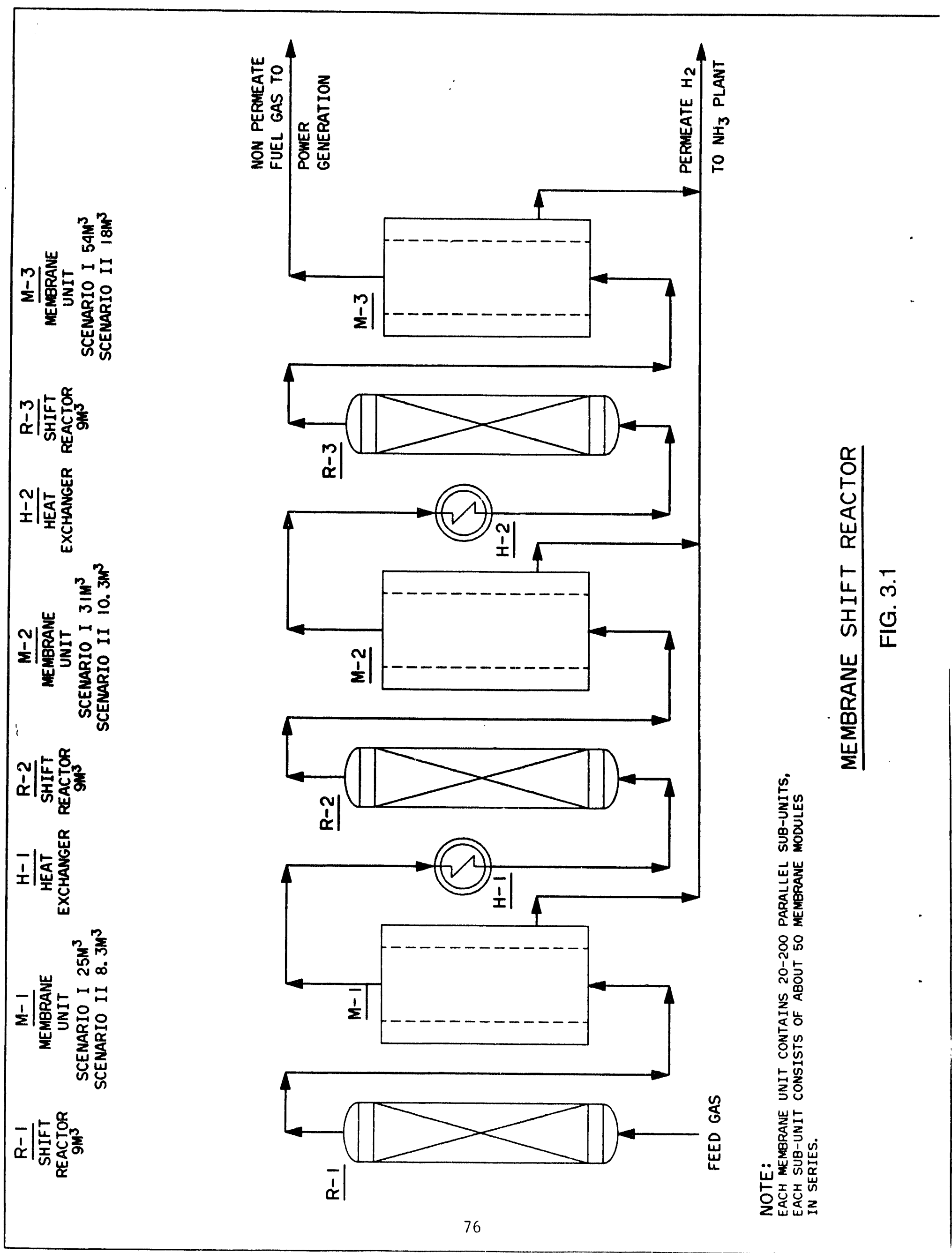




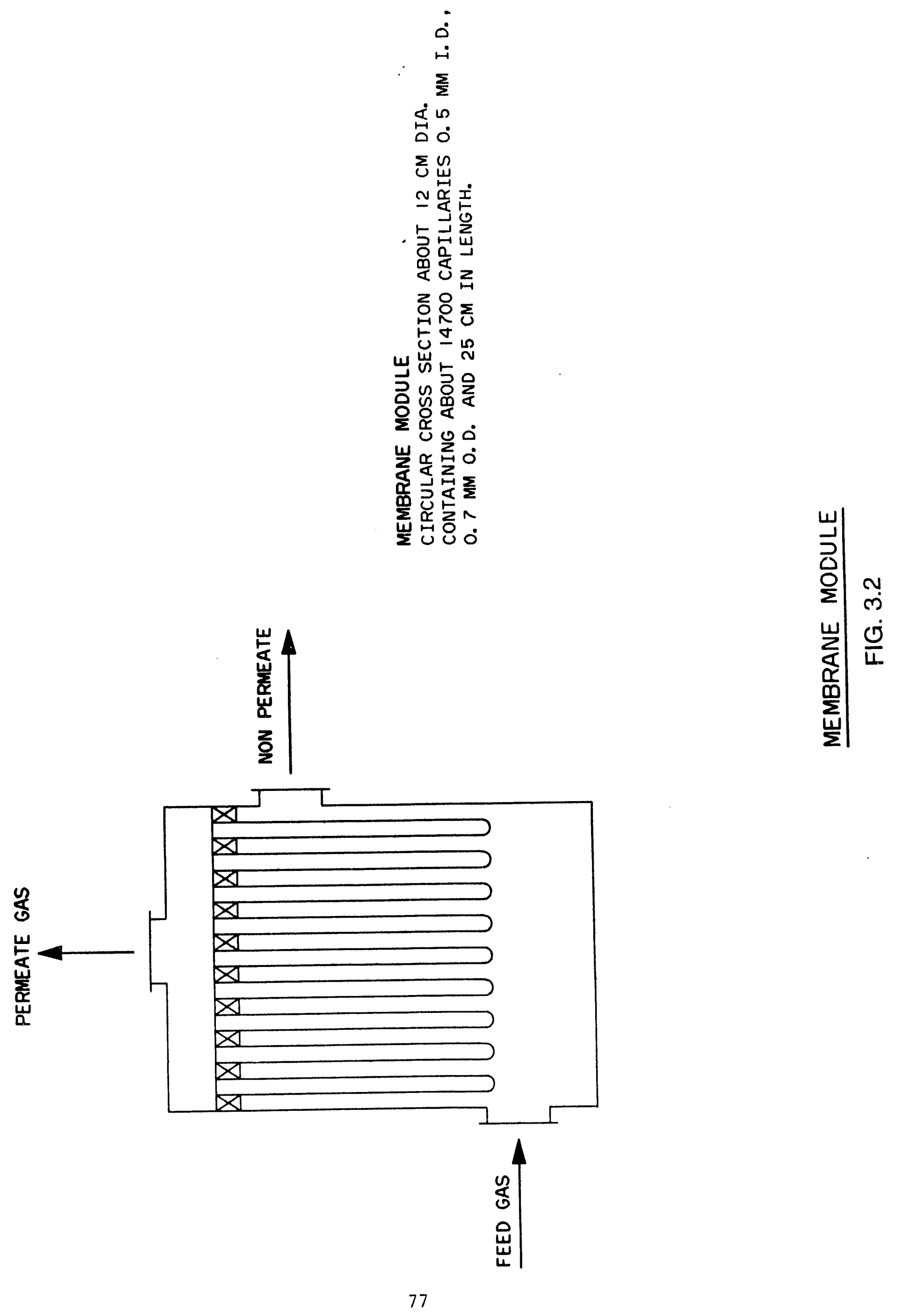




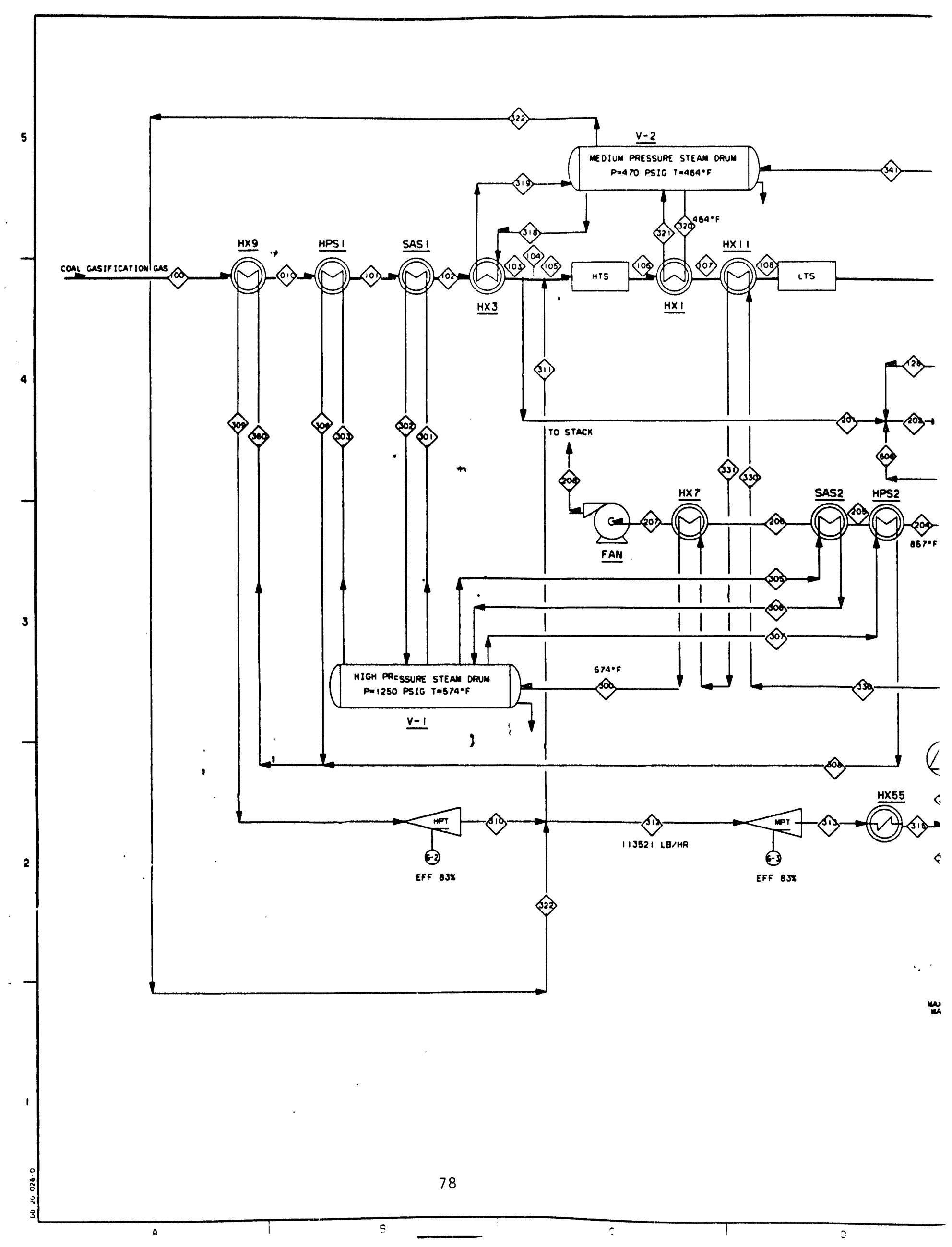




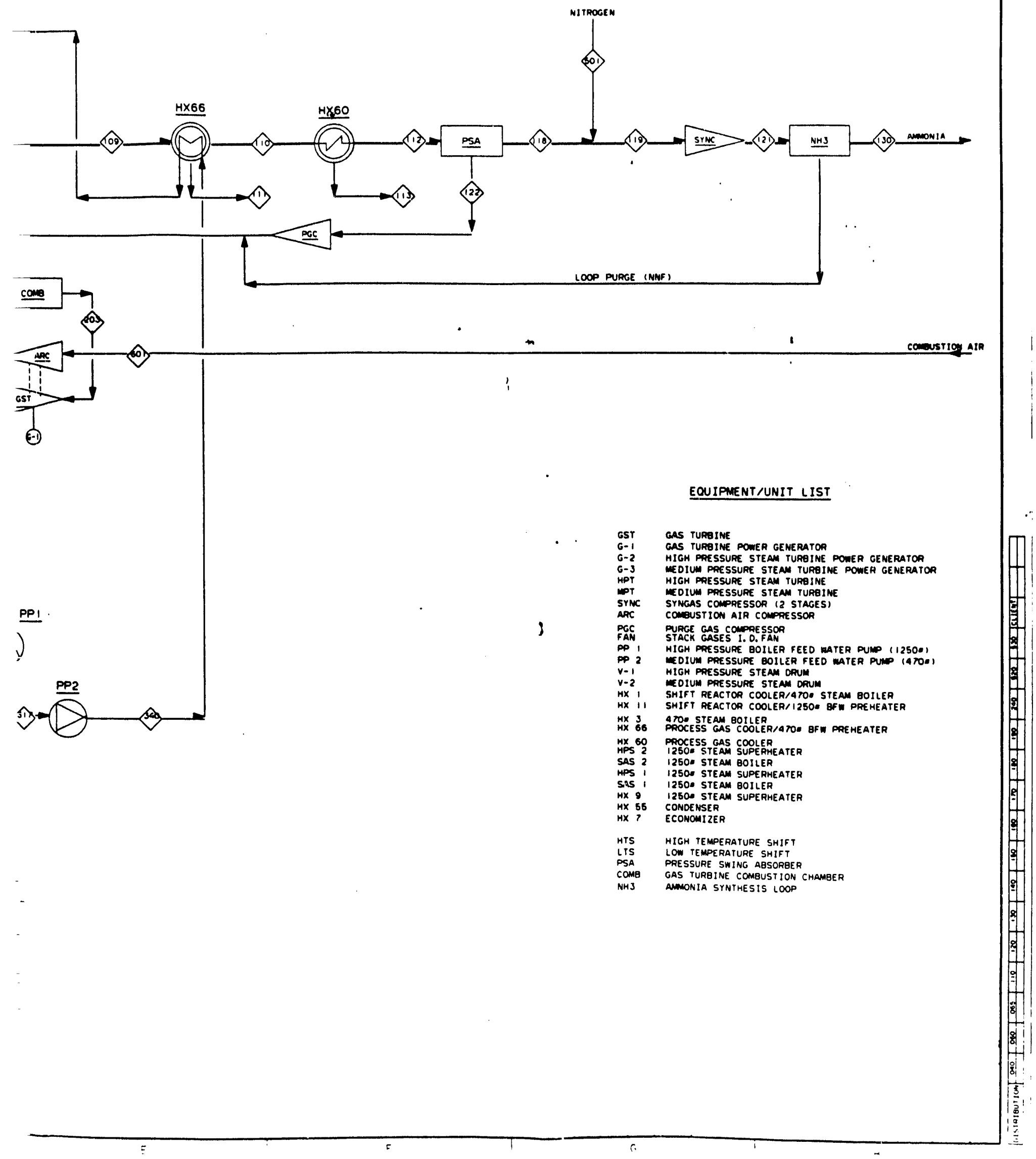




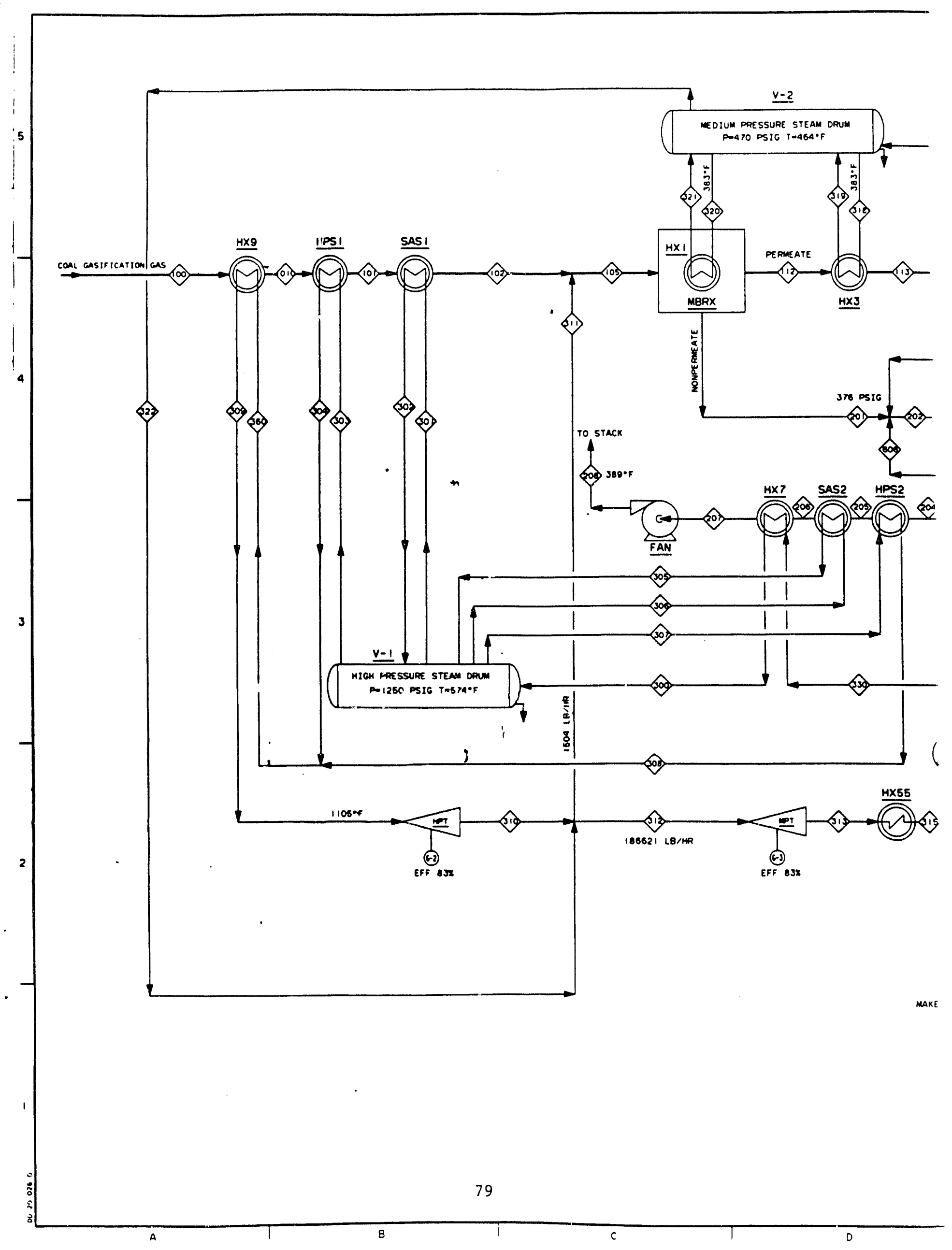




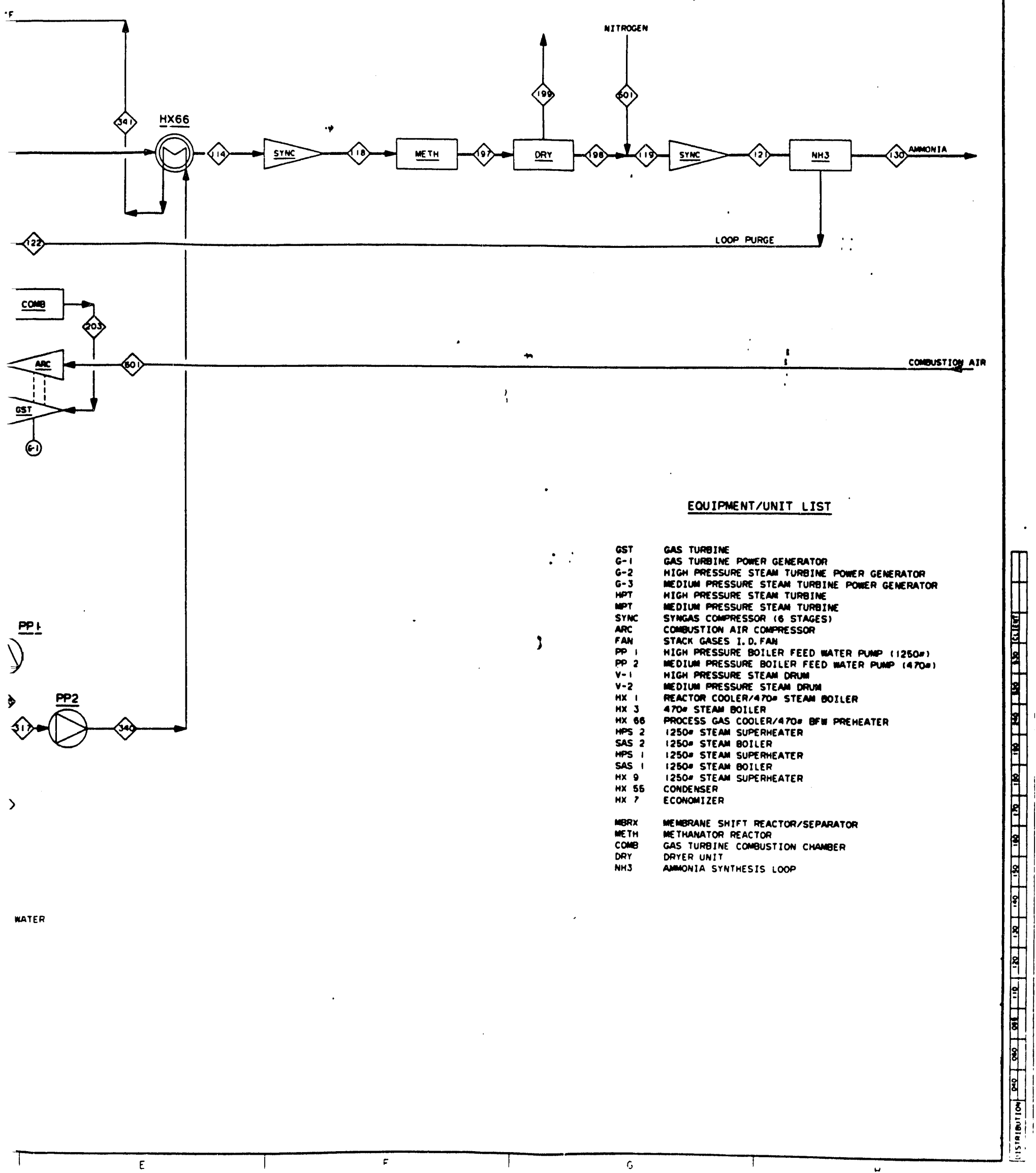




\section{Appendix}

Model of the Membrane Shift Reactor 


\section{APPENDIX. MODEL OF THE MEMBRANE SHIFT REACTOR}

\section{General Features of Membrane Reactor Design}

The membrane reactor is shown schematically in Figure 1a. It consists of one or more batteries of membrane modules connected in series and enclosed in a steel casing (the figure shows one such battery of modules). Cooling elements operating either by ordinary heat exchange or by injection of liquid water may be placed at one or more locations along the battery. The coal gas feed enters at elevated pressure into the leftmost module, flows through the series of modules and exits the last module at the right. Hydrogen permeate at about 1 atm pressure flows out of each module to a common hydrogen line.

A typical module is shown schematically in Figure 1b. It consists of a steel casing containing a large number of membrane capillaries about $200 \mu \mathrm{m}$ OD, $150 \mu \mathrm{m}$ ID and $20 \mathrm{~cm}$ length. The bottom end of each capillary is plugged, while the upper end is connected to the plenum through a glass plate. The catalyst is packed in pellet form within special modules (not containing membrane capillaries) employed in series with the membrane modules, one catalyst module every five to ten membrane modules. Alternatively (not shown in the Figure) the catalyst may be deposited on the membrane capillaries directly and thus be continuously distributed along the reactor. Each cooling element is located immediately preceding a catalyst module as shown in the Figure. But the cooling modules will generally be fewer than the catalyst modules. The shell space within each membrane module is held at a pressure of 10-20 atm in order to provide sufficient hydrogen partial pressure driving force for hydrogen permeation across the

membrane. The interior of the capillaries and the plenum is held slightly above 
atmospheric pressure to drive the flow of hydrogen to the common hydrogen line held at atmospheric pressure.

\section{Mathematical Model}

In the mathematical model described below, the membrane surface and the catalyst surface are treated as continuously and uniformly distributed along the membrane-reactor battery, rather than as being employed in discrete modules. However, heat transfer is still assumed to occur at discrete locations. Figure 2 shows this continuous mathematical model. The following notation is used in formulating the reactor equations:

\footnotetext{
$A_{\mathrm{sh}} \quad$ cross sectional area of shell, excluding capillaries $\left(\mathrm{cm}^{2}\right)$

a capillary internal radius $(\mathrm{cm})$

$a_{i} \quad$ coefficient of $c_{p i}$ (dimensionless)

$b_{i} \quad$ coefficient of $c_{p i}\left(K^{-1}\right)$

$c_{i} \quad$ coefficient of $c_{p i}\left(K^{-2}\right)$

$c_{\mathrm{pi}} \quad$ specific heat of species $\mathrm{i}(\mathrm{J} / \mathrm{mol}-\mathrm{K})$

E activation energy of $\mathrm{k}(\mathrm{J} / \mathrm{mol}-\mathrm{K})$

$E_{\mathrm{mi}} \quad$ activation energy of $\mathbf{k}_{\mathrm{mi}}(\mathrm{J} / \mathrm{mol}-\mathrm{K})$

$\mathrm{f}_{\mathrm{ci}} \quad$ total molar flowrate of species i from capillary interior to plenum (hydrogen line) for a single capillary, function of $\mathrm{z}(\mathrm{mol} / \mathrm{s})$

$\mathrm{f}_{\mathrm{c}}$ molar flowrate is capillary of species $1+2+3+4+5$

$F_{\mathrm{si}} \quad$ molar flowrate of species $\mathrm{i}$ in shell in $\mathrm{z}$-direction, function of $\mathrm{z}(\mathrm{mol} / \mathrm{s})$

FST total molar flowrate in shell, function of $\mathrm{z}(\mathrm{mol} / \mathrm{s})$

FTo total molar flowrate of feed $(\mathrm{mol} / \mathrm{s})$

$\mathrm{h} \quad$ length step in numerical integration $(\mathrm{cm})$
} 


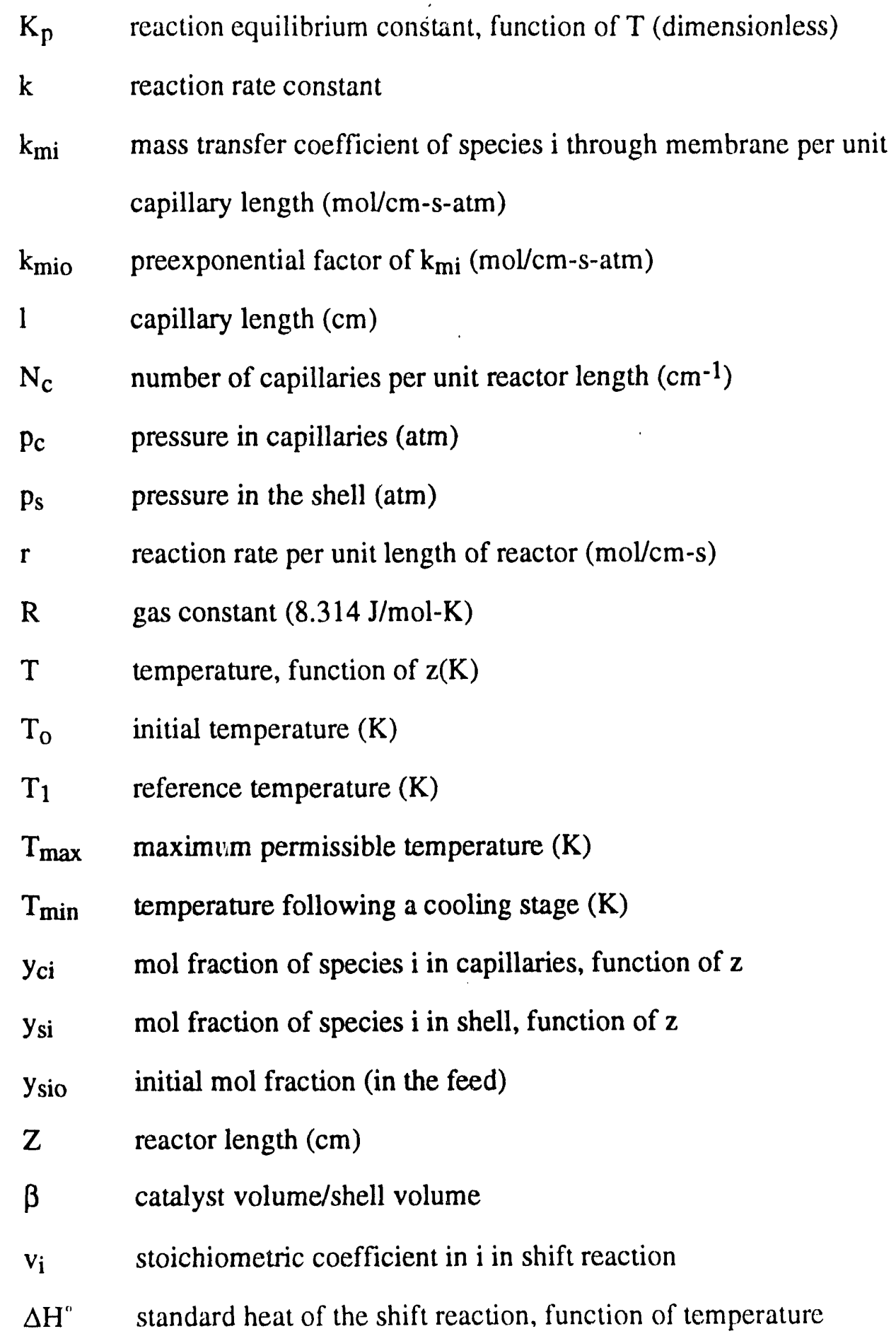


Thermodynamic Relationships

$$
\begin{gathered}
c_{p i}=R\left(a_{i}+b_{i} T+c_{i} T^{2}\right) \\
\Delta H^{\circ}(T)=H_{1}+R\left(\Delta a T+\frac{\Delta b}{2} T_{2}+\frac{\Delta c}{3} T^{3}\right)
\end{gathered}
$$

where

$$
\begin{gathered}
\Delta a=\sum v_{i} a_{i} \quad \Delta b=\sum v_{i} b_{i}, \quad \Delta_{c}=\sum v_{i} c_{i} \\
H_{1}=\Delta H\left(T_{1}\right)-R\left(\Delta a T_{1}+\frac{\Delta b}{2} T_{1}^{2}+\frac{\Delta c}{3} T_{1}^{3}\right) \\
\ln \frac{K_{p}(T)}{K(T)}=\frac{H_{1}}{R}\left(\frac{1}{T_{1}}-\frac{1}{T}\right)+\Delta a \ln \frac{T}{T_{1}} \\
+\frac{\Delta b}{2}\left(T-T_{1}\right)+\frac{\Delta c}{6}\left(T^{2}-T_{1}^{2}\right)
\end{gathered}
$$

\section{Stoichiometry}

$$
\begin{array}{cccc}
\mathrm{CO} & \mathrm{H}_{2} \mathrm{O} & = & \mathrm{H}_{2} \\
1 & 2 & \mathrm{CO}_{2} \\
1 & 4 & 4
\end{array}
$$

In addition to the four species labeled 1-4 above, we will label as species 5 all other species not participating in this reaction $\left(\mathrm{N}_{2}, \mathrm{H}_{2} \mathrm{~S}\right.$, etc.). The stoichiometric coefficients are $\mathrm{v}_{1}=-1, \mathrm{v}_{2}=-1, \quad \mathrm{v}_{3}=1, \mathrm{v}_{4}=1 \quad \mathrm{v}_{5}=0$. 


\section{Species Balances}

$$
\frac{d F_{s i}}{d z}=-N_{c} f_{c i}+v_{i} r \quad i=1, \ldots, 5
$$

where the reaction rate $r$ per unit reactor length which is the net of the forward and reverse directions is given by

$$
r=\beta A_{s h} k c_{s 1}\left(1-\frac{1}{K_{p}} \frac{y_{s 3} y_{s 4}}{y_{s 1} y_{s 2}}\right)
$$

where $c_{81}$ is the shell concentration of species 1 ; i.e. CO. This expression may be rewritten as

$$
r=k^{*} \frac{\exp (-E / R T)}{T} y_{81}\left(1-\frac{1}{K_{p}} \frac{y_{83}}{y_{81}} y_{82}\right)
$$

The capillary flowrates $f_{c i}$ are given by

$$
\begin{gathered}
f_{c i}=\mathbb{k}_{m i} p_{s i} \quad i=1,2,4,5 \\
f_{c 3}=k_{m 3}\left(p_{\mathrm{s} 3}-y_{c 3} p_{c}\right)
\end{gathered}
$$

Equation (10) incorporates the approximation of $y_{c i} \ll 1$ for $i \neq 3$, based on the high membrane selectivity for hydrogen. To eliminate $y_{c 3}$ from (11) we use

$$
y_{c 3}=\frac{f_{c 3}}{f_{c 3}+f_{c}^{\prime}} \simeq 1-\frac{f_{c}^{\prime}}{f_{c 3}}
$$

where

$$
\mathrm{f}_{\mathrm{c}}^{\prime}=\sum_{j \neq 3} \mathrm{f}_{\mathrm{cj}}
$$

so that

$$
\mathrm{f}_{\mathrm{c} 3}=\mathrm{lk}_{\mathrm{m} 3}\left(\mathrm{p}_{\mathrm{s} 3}-\mathrm{p}_{\mathrm{c}}\right)\left[1+\frac{\mathrm{f}_{\mathrm{c}}^{\prime}}{\mathrm{f}_{\mathrm{c} 3}} \frac{\mathrm{p}_{\mathrm{c}}}{\mathrm{p}_{\mathrm{s} 3}-\mathrm{p}_{\mathrm{c}}}\right]
$$


and solving for $\mathrm{f}_{\mathrm{c} 3}$ :

$$
f_{c 3}=\frac{1 k_{\mathrm{m} 3}}{2}\left(p_{s 3}-p_{c}\right)\left[\left(1+\frac{4 p_{c} f_{c}^{\prime}}{1 k_{m 3}\left(p_{s 3}-p_{c}\right)^{2}}\right)^{\frac{1}{2}}+1\right]
$$

The capillary relationship (10)-(15) incorporate the assumption of negligible pressure drop along each capillary. This assumption has been found valid for the parameter values of interest.

The partial pressures in the shell, $\mathrm{p}_{\mathrm{si}}$, are given by

$$
\begin{gathered}
p_{s i}=y_{s i} p_{s}=\frac{F_{s i} p_{s}}{F_{S T}} \\
F_{S T}=\sum F_{s j}
\end{gathered}
$$

With equations (9), (10), (15)-(17), the right hand side of Eqs. (7) functions of $T$ and $F_{s i}(i=1, \ldots, 5)$.

In addition to the basic dependent variables $T, F_{a i}$, we would like to keep track of the cumulative flowrates of the various components in the gas stream removed continuously along the reactor. The cumulative flowrates will be denoted by $F_{\text {cci }}$ and are clearly given by

$$
\mathrm{F}_{\mathrm{cci}}=\mathrm{N}_{\mathrm{c}} \int_{0}^{\mathrm{z}} \mathrm{f}_{\mathrm{ci}} \mathrm{dz} \quad \mathrm{i}=1, \ldots, 5
$$

From the computer programming standpoint it is convenient to use these equations in the differential equation form

$$
\frac{d F_{r c i}}{d_{e}}=N_{c} f_{c i}
$$

considered simultaneously with the differential equations for $F_{\text {si }}$. In the numerical calculations below we have only considered the cumulative flowrate of hydrogen 
$F_{c c 3}$ and the total cumulative flowrate $F_{c c T}=\sum F_{c c i}$, so that we can monitor the purity of the hydrogen-rich stream obtained through the membrane capillaries.

\section{Energy Balance}

$$
\left(\sum F_{s i} c_{p i}(T)\right) \frac{d T}{d z}=\left|\Delta H^{\circ}(T)\right| r
$$

It can be shown that Eq. (18) applies despite the distributed removal of gases (mainly hydrogen) through the capillaries. The reaction rate $r$ can be expressed as a function of $T$ and $F_{s i}$ by virtue of the relations already established.

\section{Initial Conditions}

These involve feed total flowrate, composition and temperature:

$$
z=0: \quad F_{S T}=F_{T_{0}} ; \quad y_{s i}=y_{s i o} ; \quad T=T_{0}
$$

\section{Reactor Cooling}

The energy balance, Eq. (18), does not include any term for cooling because . cooling is best carried out in discrete cooling stages as shown schematically in Figure 1. In the simulations below we have assumed that cooling stages will be incorporated as necessary to prevent the temperature exceeding a certain maximum value imposed by either membrane stability considerations or equilibrium considerations. This constraint is incorporated in the numerical integration of the equations by imposing the condition

$$
\begin{gathered}
\text { If } T(z)>T_{\max } \text { at some } z \text { then we set } \\
T(z+h)=T_{\text {min }}
\end{gathered}
$$


where $T_{\max }$ is the maximum permissible temperature, $h$ is the step length in the numerical integration, and $\mathrm{T}$ min is chosen as some sufficiently low starting temperature for the next reactor section, but not as low as to quench the reaction. One can optimize the location and load of the cooling stages, but this has not been attemptea in the simulations below.

\section{Numerical Solution and Sample Results}

The mathematical model was formulated as a set of 8 simultaneous ordinary differential equations for $F_{s i}(i=1, \ldots, 5), T, F_{c c T}, F_{c c 3}$. The numerical solution was obtained by $4^{\text {th }}$ order Runge-Kutta method. The following base values were chosen for the various paramiters.

$$
\begin{aligned}
& \mathrm{T}_{1}=700 \mathrm{~K} \\
& \mathrm{~K}_{\mathrm{p}}\left(\mathrm{T}_{1}\right)=9.42 \\
& \mathrm{k}_{\mathrm{mi}}=5.06 \times 10^{-7} \exp \left(-\frac{60,000}{\mathrm{kT}}\right) \frac{\mathrm{mol}}{\mathrm{cm}-\mathrm{s}-\mathrm{atm}} \mathrm{i}=1,2,4,5 \\
& \mathrm{k}_{\mathrm{m} 3}=5.50 \times 10^{-5} \exp \left(-\frac{37,000}{\mathrm{RT}}\right) \quad \frac{\mathrm{mol}}{\mathrm{cm}-\mathrm{s}-\mathrm{atm}} \\
& \mathrm{k}=5,814 \exp \left(-\frac{48,500}{\mathrm{kT}}\right) \mathrm{s}^{-1}
\end{aligned}
$$
$\mathbf{a}_{\mathrm{i}}$

$$
b_{i} \times 10^{3}
$$
$\mathrm{c}_{\mathrm{i}}$
Ysio

1

3.38

0.557

0

0.25

2

0

3

0

0.20

4

5.46

1.04

0

0.15

5

3.93

1.49

0

0.01 


$$
\begin{array}{ll}
\mathrm{N}_{\mathrm{c}} & =500 \mathrm{~cm}^{-1} \\
\mathrm{a}_{\mathrm{c}} & =0.05 \mathrm{~cm} \\
\ell & =20 \mathrm{~cm} \\
\mathrm{~A}_{\mathrm{sh}} & =400 \mathrm{~cm}^{2} \\
\mathrm{Z} & =100 \mathrm{~cm} \\
\beta & =0.01 \\
\mathrm{p}_{\mathrm{c}} & =1 \mathrm{~atm} \\
\mathrm{p}_{\mathrm{s}} & =20 \mathrm{~atm} \\
\mathrm{~F}_{\mathrm{T}_{0}} & =1 \mathrm{~mol} / \mathrm{s} \\
\mathrm{h} & =1 \mathrm{~cm} \quad \\
\mathrm{~T}_{\mathrm{o}} & =723 \mathrm{~K} \quad \text { (Figure 3), 773 (Figure 4) } \\
\mathrm{T}_{\max } & =883 \mathrm{~K} \quad \text { (Figure 3), 823 (Figure 4) } \\
\mathrm{T}_{\min } & =773 \mathrm{~K} \quad \text { (Figure 3 and Figure 4) }
\end{array}
$$

\section{Sample Calculations}

The parameters for cases 1 and 2 are listed above. The only parameters that differ among cases 1 and 2 are $T_{0}$ and $T_{\max }$ as defined above. Figure 3 shows temperature, conyersion and two mole fractions along the rear or calculated for the parameters of case 1. Only one cooling module was required causing a drop of temperature from 875 to $780 \mathrm{~K}$. The mole fraction of $\mathrm{CO}$ in the shell declines from its initial value of 0.25 to 0.047 while that of $\mathrm{H}_{2}$ in the shell goes from 0.2 to 0.053 . The mol fraction of hydrogn in the hydrogen product stream is 0.996 . There 
is less than $0.5 \%$ of other gase $\left(\mathrm{CO}, \mathrm{H}_{2} \mathrm{O}, \mathrm{CO}_{2}\right)$ in that stream, which is one of the advantages of the membrane reactor. The conversion can be defined in two way:

$$
\begin{aligned}
& X_{1}=\frac{F_{\mathrm{sCO}}(0)-F_{\mathrm{sCO}}(Z)}{F_{\mathrm{sCO}}(0)} \\
& X_{2}=\frac{F_{\mathrm{CCH}_{2}}(Z)}{F_{\mathrm{sCO}}(0)+F_{\mathrm{sH}_{2}}(0)}
\end{aligned}
$$

The first definition is based on CO and is the customary definition for conversion of an ordinary reactor. It neglects the very small amount of $\mathrm{CO}$ going through the membrane to the hydrogen stream. The second definition is based on the hydrogen separated in almost pure form as a fraction of the total potential hydrogen in the feed $\left(\mathrm{H}_{2}+\mathrm{CO}\right)$. It is a more meaningful measure of the combined reaction- separation performance of the membrane reactor. From the numerical results we obtained $\mathrm{X}_{1}$ $=0.886, \mathrm{X}_{2}=0.865$. For the same feed conditions, a conventional shift reactor operating with one cooling stage would yield equilibrium conversion 0.656 .

The results of the second case study are shown in Figure 4. Because of the lower $T_{\max }$, this reactor includes for cooling stages yielding conversions $\mathrm{X}_{1}=0.90$, $\mathrm{X}_{2}=0.860$. The results are not too difficult from those of case 1 .

To evaluate the performance of the membrane reactor it is necessary to take into account the purity of the hydrogen stream, the possible requirements for removing the $\mathrm{H}_{2} \mathrm{~S}$ content in that stream, the size of the stream going through acid gas removal, the size of the recycle stream and other factors relative to the corresponding performance of a conventional reactor. These comparisons are within the scope of Task 4, economic analysis to be carried out by KTI corporation. 

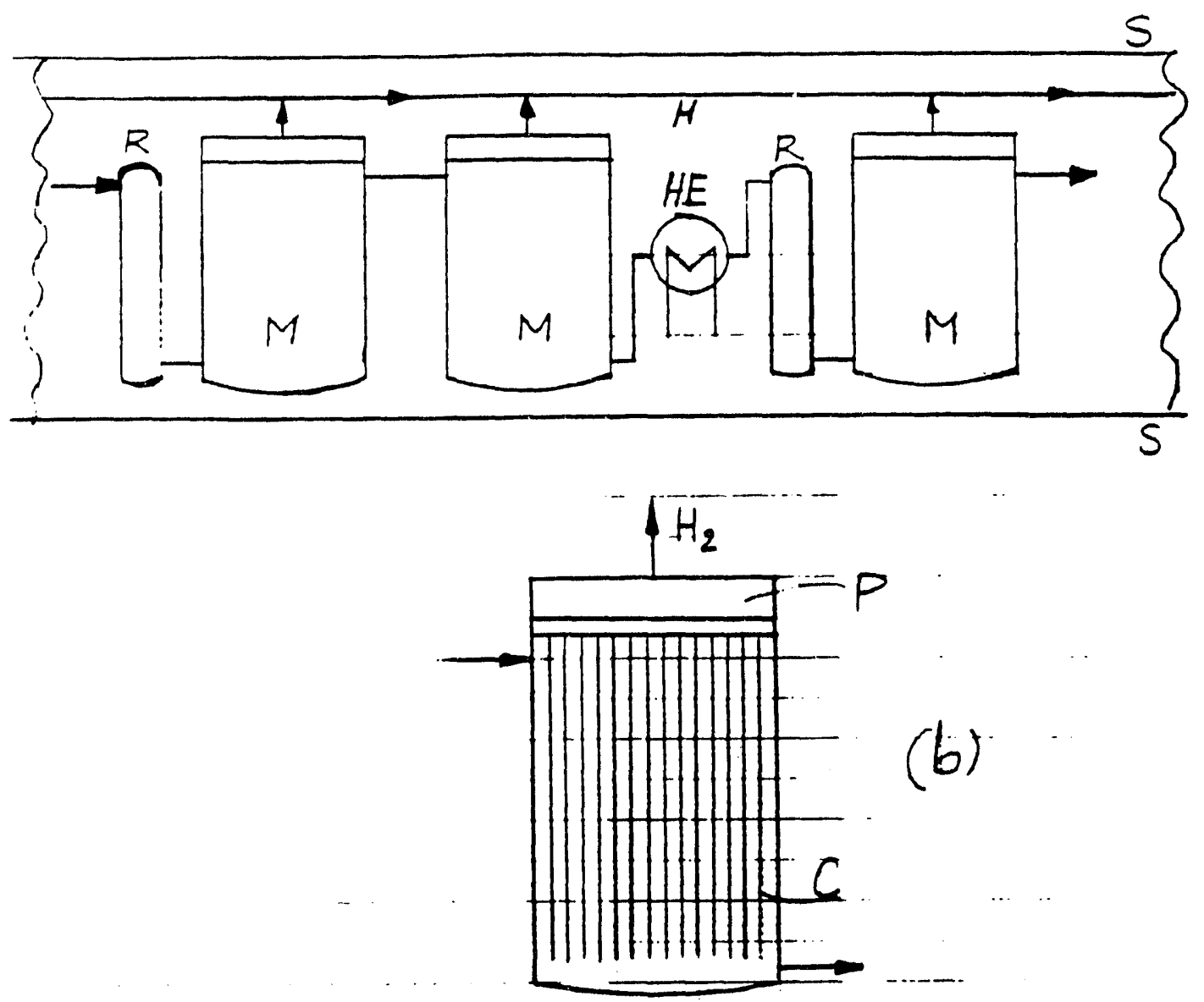

Figure 1. Schematic of a section of a membrane reactor (a) and a membrane module (b). M: membrane module; $R$ : catalyst module; HE: heat exchange module; $H$ : hydrogen line; $S$ : reactor shell; P: plenum; C: membrane capillary. 


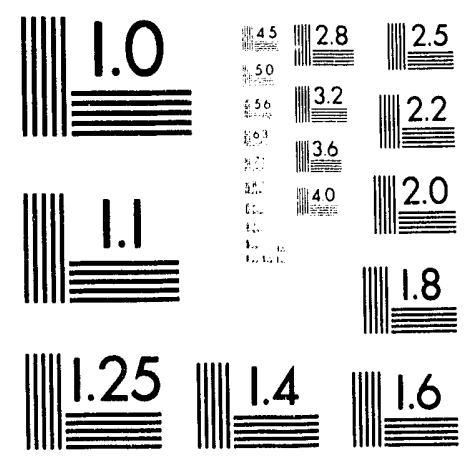



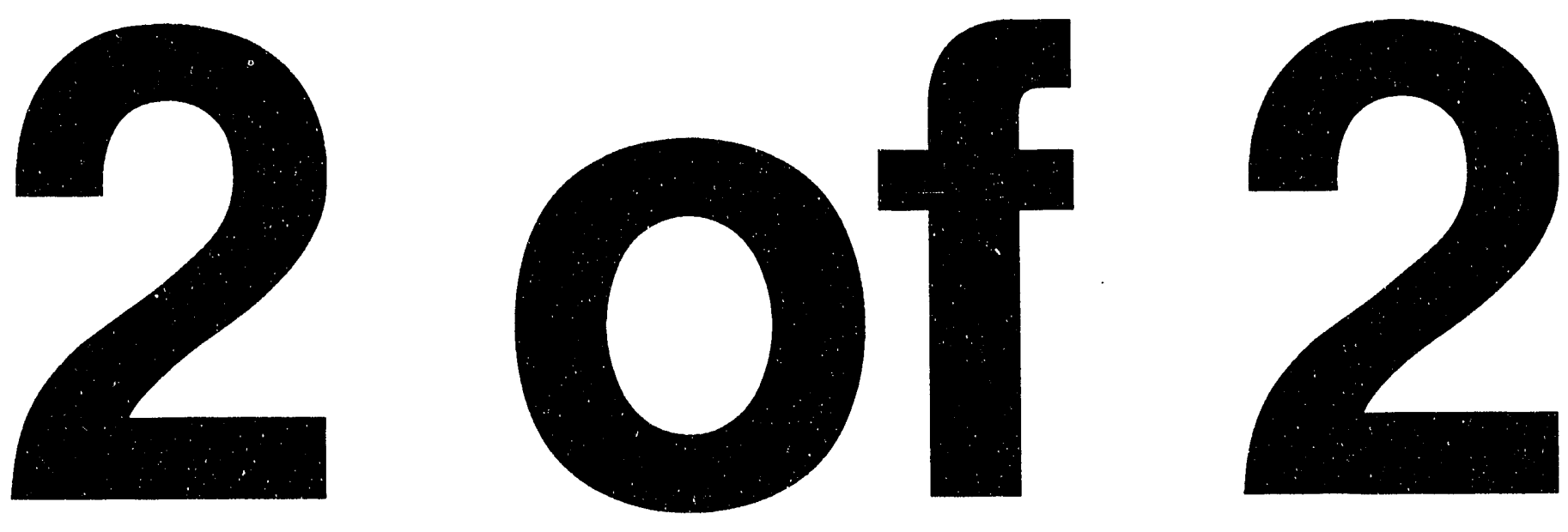


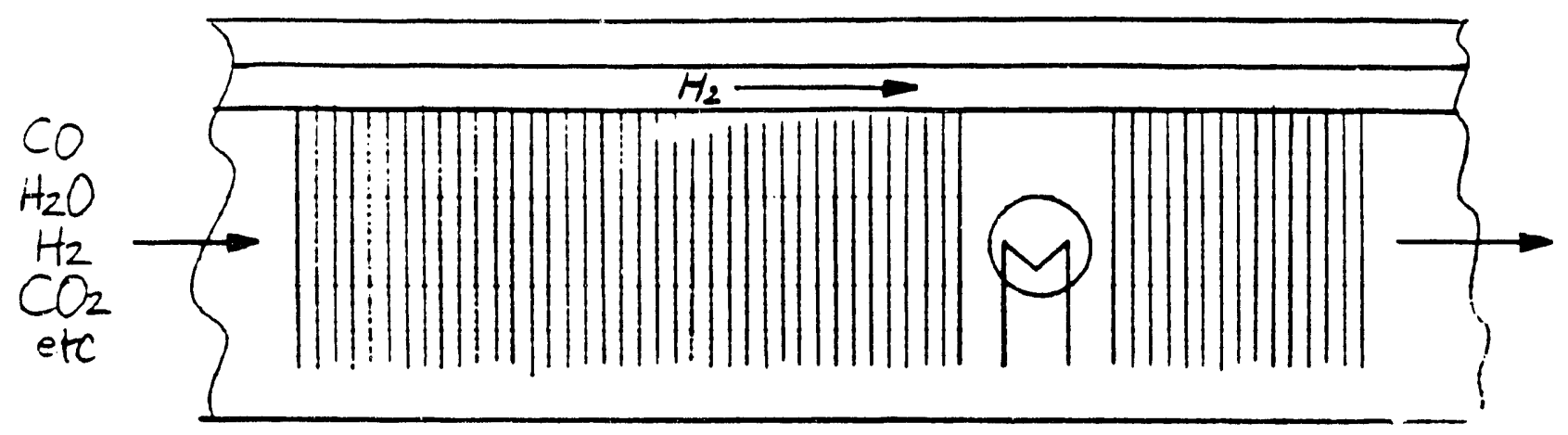

Figure 2. Schematic of the membrane reactor model assuming uniformly distributed catalyst and membrane area, discrete cooling steps. 


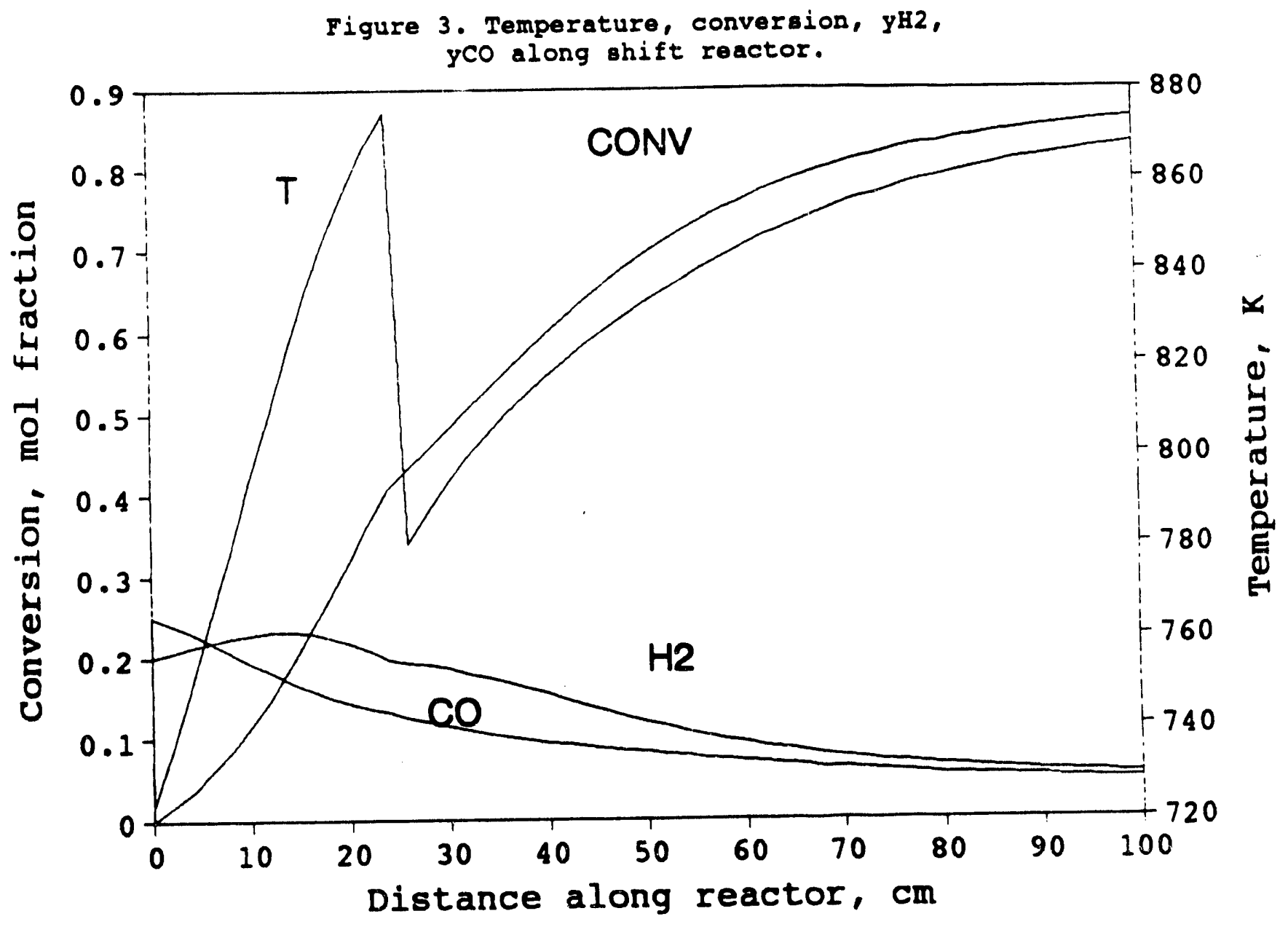




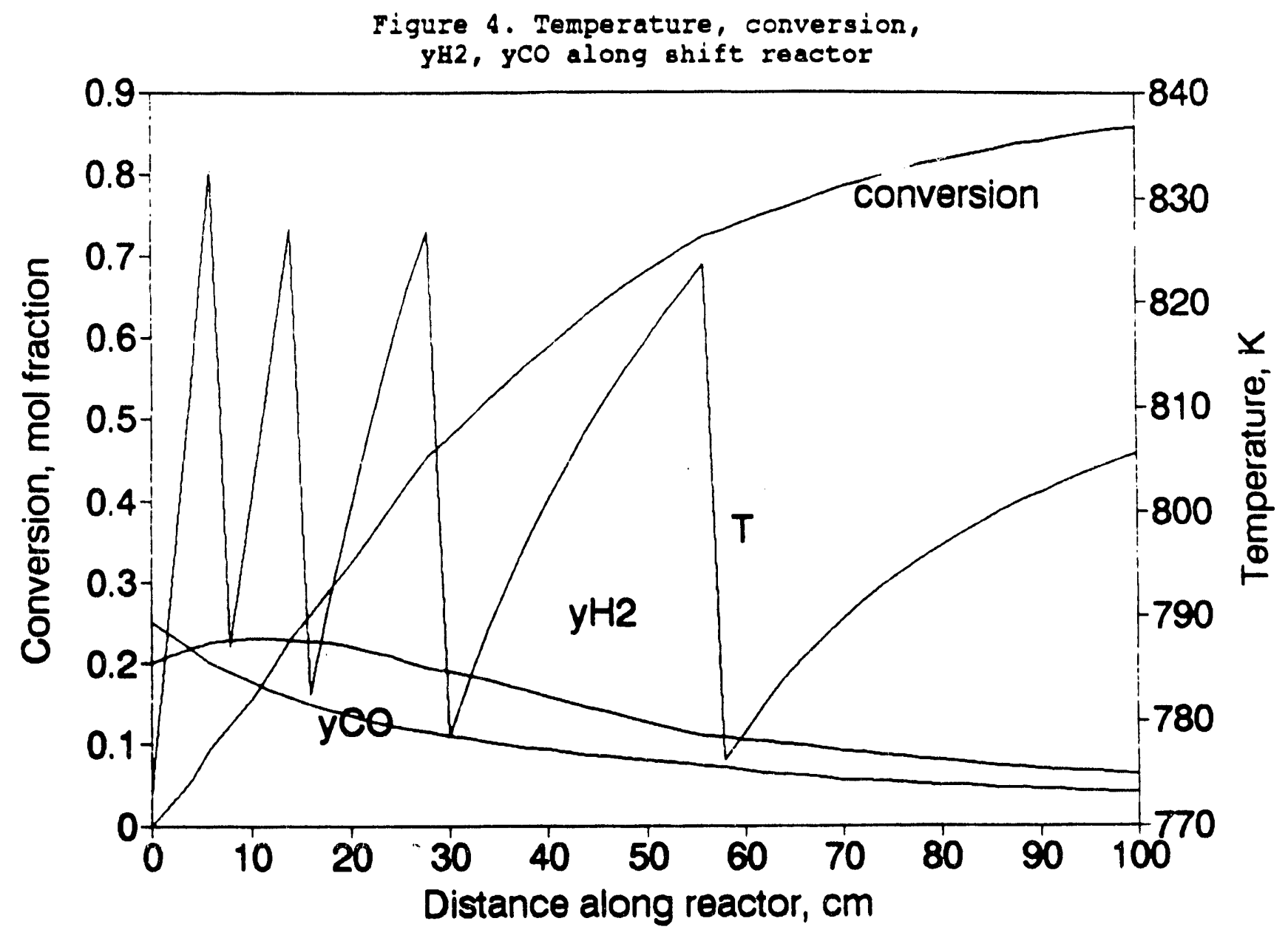


Chapter 4

Conclusions 
1. This project has demonstrated the feasibility of preparing membranes highly permselective to hydrogen by CVD of dense $\mathrm{SiO}_{2}, \mathrm{TiO}_{2}, \mathrm{Al}_{2} \mathrm{O}_{3}$ and $\mathrm{B}_{2} \mathrm{O}_{3}$ layers within the wall of porous Vycor tubes. The $\mathrm{SiO}_{2}$ membranes were superior in hydrogen permeance and selectivity, followed by the $\mathrm{TiO}_{2}$ membranes. The $\mathrm{Al}_{2} \mathrm{O}_{3}$ membranes had permeance and selectivity considerably below those of the $\mathrm{TiO}_{2}$ membranes. The $\mathrm{B}_{2} \mathrm{O}_{3}$ membranes showed some interesting permeation properties but failed upon heating after prolonged exposure to humid laboratory air.

2. Alumina and titania membranes could be prepared only by opposing reactants deposition. Silica and boria membranes could be produced by one-sided deposition as well as by opposing reactants deposition. The silica membranes prepared by onesided deposition had higher hydrogen permeance but lower selectivity than those prepared by opposing reactants deposition.

3. Annealing of the membranes in dry nitrogen at $700-750^{\circ} \mathrm{C}$ for 12 hours reduced slightly $(0-10 \%)$ the permeance of hydrogen and reduced substantially the permeance of nitrogen thus improving the hydrogen selectivity. However, upon extended hydrothermal exposure to $550^{\circ} \mathrm{C}$ and 3 atmospheres of water vapor for two to three weeks, the hydrogen permeance declined by a factor of 3-5 to a new seemingly stable level. This decline is evidently due to an increase of activation energy for diffusion accompanying the transformation of the highly defective initial deposit layer to a structure similar to that of Vycor glass. The permeance to nitrogen underwent a similar decline such that selectivity was not significantly changed by the hydrothermal treatment. During stability testing the silica membranes were also subjected to twice or three times to cooling and reheating, from $550^{\circ} \mathrm{C}$ to room temperature and back to $550^{\circ} \mathrm{C}$. Membranes prepared by opposing reactants deposition or by one-sided deposition using large pressures of $\mathrm{SiCl}_{4}$ often developed 
cracks during these repeated temperature changies. By contrast, membranes prepared by two-sided deposition using $\mathrm{SiCl}_{4}$ pressures lelow $0.1 \mathrm{~atm}$ were stable to repeated cooling and reheating. The most favorable conditions for silica membrane preparation were about $750^{\circ} \mathrm{C}$ temperature, small $\mathrm{SiCl}_{4}$ pressure (e.g. $0.02 \mathrm{~atm}$ ) and relatively high $\mathrm{H}_{2} \mathrm{O}$ pressure $(0.05 \mathrm{~atm}$ or higher). The membranes prepared under these conditions had stable hydrogen permeance (after 2-3 weeks of hydrothermal treatment) of about $0.1 \mathrm{~cm}^{3}(\mathrm{STP}) / \mathrm{cm}^{2}$-min-atm at $500^{\circ} \mathrm{C}$.

4. An evaluation of the commercial potential of the silica membranes was carried out with reference to an ammonia-from-coal process. A conventional and a membraneassisted process with the same consumption of coal and production of ammonia were compared. The two processes differed only in the amount of electrical power cogenerated. Because of higher efficiency the membrane process had higher net power generation. An economic evaluation of the extra power generated versus the extra capital cost of the membrane process required data on the cost of Vycor tubing and module fabrication. Reliable data are not available at this time because Vycor tubing of the desired dimensions is not commercial and module fabrication has not yet been demonstrated. Only some crude estimates could be made. These estimates suggest that with the currently achievable hydrogen permeance (stabilized value) and with the price of electricity in the range of $2 \mathrm{c}-5 \mathrm{c} / \mathrm{kWh}$ the extra cost of the membrane process would be recovered in 5-13 years. This recovery period is too long. If the membrane permeance could be increased by a factor of 3 , or the cost of Vycor tubing reduced by the same factor, the extra capital cost of the membrane process would be recovered in only $0.6-1.5$ years which would make the process competitive. 
5. The experience gained from the membrane preparation experiments and from parallel mathematical modeling studies of the deposition process suggest that the hydrogen permeance can be significantly increased by certain variations of the basic deposition process. The most straightforward of these variations is to use a more reactive silica precursor. Using support tubes with smaller pores and thinner walls, or composite tubes having a thin surface layer of the smaller pores is another possible means of achieving higher permeance. In parallel with a program to increase membrane permieance, it is essential to develop module fabrication techniques. These two efforts can then be followed by longer stability testing at a larger scale than is possible with single membrane tubes. When progress has been made in these two directions it should be possible to obtain more accurate cost estimates for economic evaluation. 

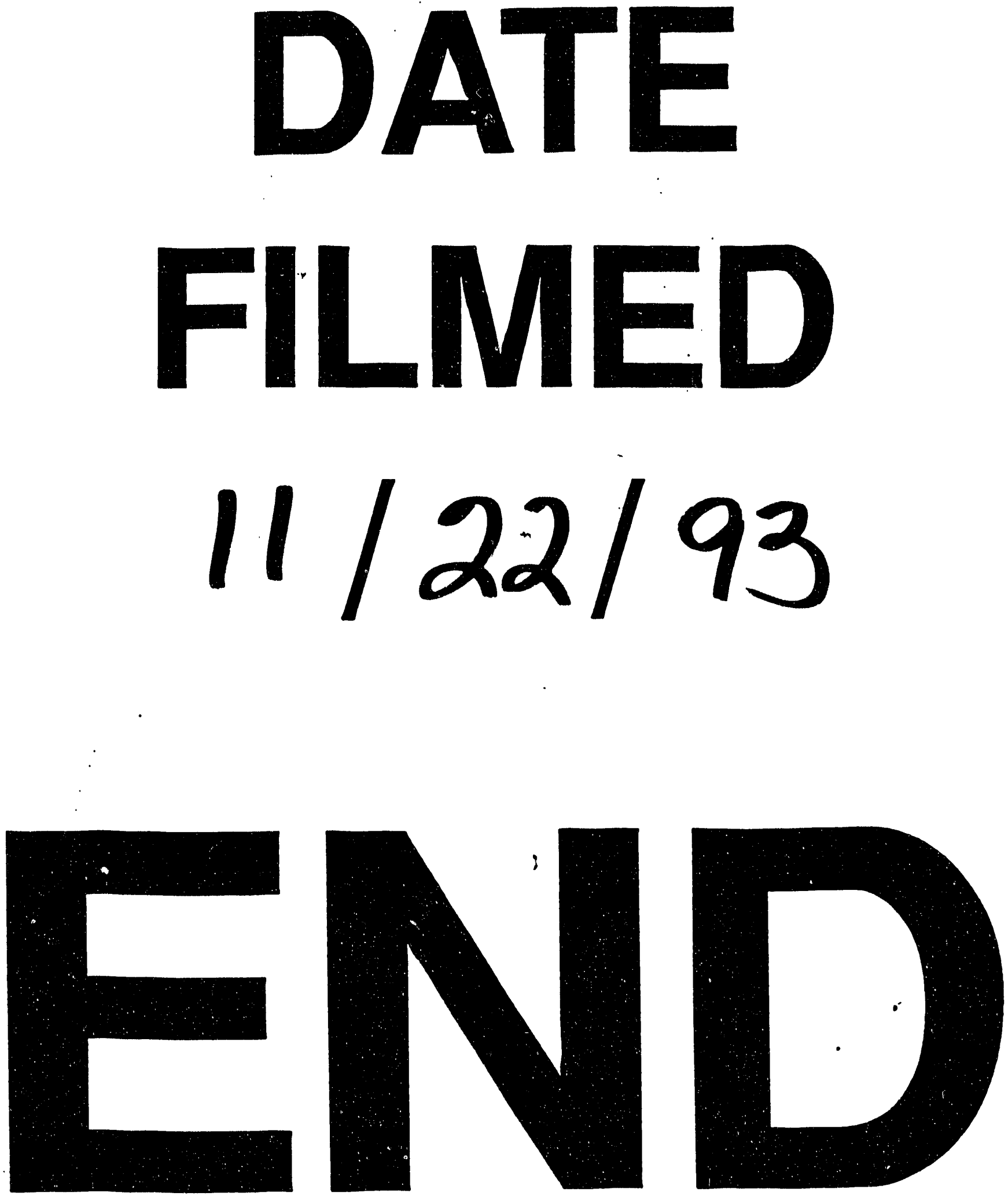
